\title{
THE COMET HALLEY DUST AND GAS ENVIRONMENT
}

\author{
NEIL DIVINE ${ }^{1}, H$. FECHTIG ${ }^{2}$, T. I. GOMBOSI ${ }^{3}{ }^{4}$, M. S. HANNER ${ }^{1}$, \\ H. U. KELLER ${ }^{5}$, S. M. LARSON ${ }^{6}$, D. A. MENDIS ${ }^{7}$, RAYL. NEWBURN, JR. ${ }^{1}$, \\ R. REINHARD ${ }^{8}, Z$. SEKANINA ${ }^{1}$, and D.K. YEOMANS ${ }^{1}$
}

(Received 19 November, 1985)

\begin{abstract}
Quantitative descriptions of environments near the nucleus of comet $P /$ Halley have been developed to support spacecraft and mission design for the flyby encounters in March, 1986. To summarize these models as they exist just before the encounters, we review the relevant data from prior Halley apparitions and from recent cometary research. Orbital elements, visual magnitudes, and parameter values and analysis for the nucleus, gas and dust are combined to predict Halley's position, production rates, gas and dust distributions, and electromagnetic radiation field for the current perihelion passage. The predicted numerical results have been useful for estimating likely spacecraft effects, such as impact damage and attitude perturbation. Sample applications are cited, including design of a dust shield for spacecraft structure, and threshold and dynamic range selection for flight experiments. We expect that the comet's activity may be more irregular than these smoothly varying models predict, and that comparison with the flyby data will be instructive.
\end{abstract}

\section{Table of Contents}

1. Introduction

2. Orbital elements and nongravitational forces

2.1. Nongravitational forces and comet Halley

2.2. Recent orbital work on comet Halley

3. Overview of cometary activity

3.1. The semi-empirical photometric theory

3.2. Predictions for Halley in 1986

4. Parameters for the nucleus

4.1. Composition, structure, and density

4.2. Albedo, size, and mass

4.3. Rotation period and axis

5. Nucleus-coma interface region

5.1. Surface temperatures and gas production

5.2. Gas-dust interaction and terminal velocities

6. Cometary Gas

6.1. The chemical species

6.2. Chemical reactions

6.3. Distributions of gas molecules

6.4. A gas model for Halley

1 Jet Propulsion Laboratory, California Institute of Technology, Pasadena, California 91109, U.S.A.

2 Max-Planck-Institut für Kernphysik, D-6900 Heidelberg, F.R.G.

3 Space Physics Laboratory, University of Michigan, Ann Arbor, Michigan 48109, U.S.A.

4 Central Research Institute for Physics, Hungarian Academy of Sciences, Budapest, Hungary.

5 Max-Planck-Institut für Aeronomie, D-3411 Katlenburg-Lindau, F.R.G.

${ }^{6}$ Lunar and Planetary Laboratory, University of Arizona, Tucson, Arizona 85721, U.S.A.

7 Electrical Engineering and Computer Sciences Department, University of California at San Diego, La Jolla, California 92093, U.S.A.

${ }^{8}$ Space Science Department, European Space Research and Technology Centre, 2200 AG Noordwijk, The Netherlands. 
7. Cometary dust

7.1. Optical and infrared properties

7.2. Size distribution

7.3. Composition, structure, and bulk density

7.4. Dust concentration in the coma and tail

8. Cometary active regions

8.1. Photographs of Halley and their image processing

8.2. Descriptions of features and their sources on the surface

8.3. Modeling the evolution of dust features for Halley

8.4. Enhancement of dust concentration in jets

9. Radiation model

9.1. Radiation from cometary gas

9.2. Resonance radiation of hydrogen

9.3. Radiation from the dust

9.4. Visibility of the nucleus

9.5. Center-of-light/center-of-mass offset

10. Applications

10.1. Adaptation of the models

10.2. Consequences for spacecraft and missions

10.3. Consequences for experiments on flyby spacecraft

11. Range of uncertainties and effects not included

12. Summary

Acknowledgments

Appendix A. Parameters and assumptions for model HALLEY 85-4

Appendix B. Dust dustributions for flyby missions

\section{Introduction}

In the history of scientific inquiry into the phenomena exhibited by Periodic Comet Halley, three events represent major landmarks. First among these was the demonstration by Halley (1705) that three comet apparitions (in the years 1531, 1607, and 1682) in fact represented a single object obeying the same celestial mechanics as the planets. His successful prediction of the circumstances of the 1759 return was rewarded by the naming after him of what has since become the most famous of all comets, for which recorded observations now span over two millennia (Yeomans and Kiang, 1981). The predictability of $P /$ Halley's motion and exceptionally rich display of cometary phenomena are the major drivers for spacecraft experiments now en route to this comet's vicinity.

Second among these events was the publication by Bobrovnikoff (1931) of a comprehensive review of data acquired during the 1909 to 1911 apparition (when $P /$ Halley's formal label was Comet 1910 II). These data include magnitudes, photographs, spectra and other measurements, and represent a guide to the major quantitative foundation for the prediction of Halley's specific properties in the present decade (although some magnitude estimates from the 1835 and earlier apparitions are also relevant). Additionally the successes and failures of Bobrovnikoff's work as a data archive serve as an inspiration to the International Halley Watch (IHW), whose goals 
include the advocacy, coordination, standardization and archiving of modern data (for which $P /$ Halley has the temporary label Comet $1982 \mathrm{i}$ ).

Third among these events was the development by Whipple $(1950,1951)$ of the icy conglomerate model for a cometary nucleus. The concepts advanced in these seminal papers form the basis for quantitative analysis both of the nongravitational contributions to cometary dynamics and of the diverse phenomena which generate the gas, dust and plasma environments observed from Earth in $P / \mathrm{Halley}$ and other comets. An accessible review of the advance in cometary science provided by this model may be found in Whipple (1976).

To enable the spacecraft-borne experiments (which will encounter $P / \mathrm{Halley}$ in March, 1986) to set a fourth landmark in the scientific investigation of this unique member of the solar system, both engineering and scientific decisions have needed the support of environmental models for the comet's immediate vicinity. For this purpose the InterAgency Consultative Group (IACG), an international forum for cooperation among the spacedraft management agencies and the IHW (Reinhard, 1984), sponsors Working Group 1 (WG-1) on the Halley environment (excepting plasma science, handled by WG-2). The present review, whose authors are all affiliated with WG-1, represents the consensus status of the dust, gas and radiation environments as predicted in 1985 and evaluated for $P /$ Halley in March, 1986.

Although some elements of the environmental descriptions developed in the following sections have been part of our scientific understanding of cometary physics for many years, the potential for direct exploration of comets by spacecraft has provided the impetus for their assembly into consistent and comprehensive numerical models. An early example is provided by Taylor et al. (1973), when comet $P /$ Encke was a possible target for a slow flyby mission. The first application of such modeling for $P / H$ alley was developed by Newburn and Yeomans (1977), and this work was expanded (e.g., Newburn, 1979, 1981; and other articles in the same volumes) as Halley missions became fashionable and as the severity of the micrometeoroid threat from cometary dust became clear. Recently computer capabilities have been harnessed for the numerical modeling tasks, as exemplified by the results of Divine (1981a), Hellmich and Keller (1981b), Divine and Newburn (1983), and others for the dust. Eventually gas and radiation environments were included in the numerical modeling activities as well (e.g., Sections 5, 6, and 9 below; Divine, 1981b; Hellmich and Keller, 1981a), and the relevance of modeling for specific dust features (culminating in the work of Sekanina, as represented by several entries in the reference list) was also appreciated.

Finally, the race to transport a complement of spacecraft-borne experiments to Halley's vicinity for remote and in-situ investigations narrowed to the five vehicles identified in Table I. Additional details for VEGA-1 and VEGA-2, both VENERAclass, three-axis stabilized spacecraft (which will inject probes into Venus' atmosphere en route to Halley), are provided by Sagdeev et al. (1982) and Gombosi (1985). For SAKIGAKE and SUISEI (formerly MS-T5 and PLANET-A), both spin-stabilized spacecraft intended for more remote encounters at Halley, they are provided by Hirao (1984). For GIOTTO, a GEOS-class, spin-stabilized spacecraft carrying protection 
TABLE I

Trajectory summary for five Halley flyby spacecraft, arranged in order of launch date

\begin{tabular}{lrlllll}
\hline Vehicle & Launch date & $\begin{array}{l}\text { Encounter } \\
\text { date }\end{array}$ & $\begin{array}{l}\text { Heliocentric } \\
\text { distance } \\
(\mathrm{AU})\end{array}$ & $\begin{array}{l}\text { Min. appr. Appr. } \\
\text { dist. } \\
(\mathrm{km})\end{array}$ & $\begin{array}{l}\text { Relative } \\
\text { phase } \\
\text { angle } \\
\text { (deg) }\end{array}$ & $\begin{array}{l}\text { velocity } \\
\left(\mathrm{km} \mathrm{s}^{-1}\right)\end{array}$ \\
\hline VEGA-1 & 15 Dec., 1984 & 6 Mar, 1986 & 0.785 & 10000 & 111 & 79 \\
VEGA-2 & 21 Dec., 1984 & $9 \mathrm{Mar}, 1986$ & 0.827 & 3000 & 113 & 77 \\
SAKIGAKE & 8 Jan., 1985 & $11 \mathrm{Mar}, 1986$ & & $7 \times 10^{6}$ & 109 & 75 \\
$\begin{array}{l}\text { GIOTTO } \\
\text { SUISEI }\end{array}$ & 2 July, 1985 & $14 \mathrm{Mar}, 1986$ & 0.885 & 500 & 107 & 68 \\
\hline
\end{tabular}

suitable for a daringly close cometary encounter, they are provided by Reinhard (1981a, b) and other articles in the same volumes. The full complement of experiments to investigate Halley's environment is reviewed by Blamont (1983), and other articles in the same volume describe the cameras, spectrometers, dust counters and analysers, plasma and magnetic field instruments, and other devices included in this unique spacecraft fleet.

\section{Orbital Elements and Nongravitational Forces}

Prediction of the present and future position of Comet $P /$ Halley requires the application both of standard techniques for cometary astrometry and ephemerides (e.g., Marsden and Roemer, 1982) and of specialized analysis which takes advantage of Halley's unique observational history (Yeomans and Kiang, 1981). The following sections describe the role of nongravitational forces in this work and present a current set of orbital elements and parameters for ephemeris use during the 1986 apparition.

\subsection{NongRaVITATIONAL FORCES AND COMET HalleY}

Beginning with the work of Bessel $(1835,1836 \mathrm{~b})$, it became clear that the motion of $P /$ Halley was influenced by more than the solar and planetary gravitational accelerations. Michielsen (1968) pointed out that perihelion passage time predictions that had been based strictly on gravitational perturbation calculations required a correction of + 4.4 days for each of several revolutions. Kiang (1971) determined a mean correction of +4.1 days.

In introducing the icy conglomerate model for a cometary nucleus (cf. Section 4), Whipple $(1950,1951)$ recognized that comets may undergo substantial perturbations due to reactive forces or rocket-like effects acting upon the cometary nucleus itself. In an effort to represent accurately the motions of many short period comets, Marsden $(1968,1969)$ modeled the nongravitational forces with radial and transverse acceleration terms in the equations of motion. Marsden et al. (1973) modified the nongravitational terms to represent the vaporization of water ice as a function of heliocentric distance $r$ (sample units AU). In this approach the equations of motion specify the cometary 
acceleration $\left(\mathrm{AU}\right.$ day $\left.^{-2}\right)$ in the form

$$
\frac{\mathrm{d}^{2} \mathbf{r}}{\mathrm{d} t^{2}}=-\frac{G M_{\odot}}{r^{2}} \hat{\mathbf{r}}+\frac{\partial R_{p}}{\partial \mathbf{r}}+g_{n}\left(A_{1} \hat{\mathbf{r}}+A_{2} \hat{\mathbf{t}}\right) .
$$

Here $G M_{\odot}\left(\mathrm{AU}^{3}\right.$ day $\left.^{-2}\right)$ is the product of the gravitational constant and the solar mass, $R_{p}\left(\mathrm{AU}^{2} \mathrm{day}^{-2}\right)$ is the planetary disturbing function, and the nongravitational forces are modeled using the dimensionless function

$$
g_{n}=\frac{\alpha_{n}\left(r_{s} / r\right)^{m_{n}}}{\left[1+\left(r / r_{s}\right)^{n_{n}}\right]^{k_{n}}}
$$

which has the value 1.0 at $r=1.0 \mathrm{AU}$. The scale distance $r_{s}$ is the heliocentric distance beyond which reradiation of the solar energy dominates its use for vaporizing the comet's nuclear ices. For water ice, appropriate values for the parameters in Equation (2.2) are $r_{s}=2.808 \mathrm{AU}, m_{n}=2.15, n_{n}=5.093, k_{n}=4.6142$, and $\alpha_{n}=0.111262$.

If the comet's nucleus were not rotating, the outgassing would always be preferentially toward the Sun and the resulting nongravitational acceleration would act only in the anti-solar direction. However, the rotation of the nucleus, coupled with a thermal lag between the subsolar point and the point of maximum outgassing on the nucleus' surface, introduces a transverse acceleration component in either the direction of the comet's motion or opposite to it (depending on the sense of rotation). The radial unit vector $\hat{\mathbf{r}}$ is defined outward along the Sun-comet vector, while the transverse unit vector $\hat{\mathbf{t}}$ is directed normal to $\hat{\mathbf{r}}$ (in the orbit plane) and in the direction of the comet's orbital motion. An acceleration component normal to the orbit plane is certainly present for most comets, but its periodic nature makes detection difficult in these computations because we are solving for an average nongravitational acceleration over three or more apparitions. While the nongravitational acceleration term (Equation (2.2)) was originally formulated for water ice, Marsden et al. (1973) have shown that if the Bond albedo in the visible range equals the infrared albedo, then the scale distance $r_{s}$ is inversely proportional to the square of the heat of vaporization for the sublimating molecule.

Using observations of $P /$ Halley over the interval 1607-1911, Yeomans (1977) applied a least squares differential correction process to solve for the six initial orbital elements and the two nongravitational parameters $A_{1}$ and $A_{2}$ (Equation (2.1)). Different values for the scaling distance were tried with the result that $r_{s}=2.808 \mathrm{AU}$ is close to the optimum value. This suggests that the nongravitational forces acting on $P / \mathrm{Halley}$ are consistent with the vaporization of water ice. This result is a general one governing nearly all comets for which nongravitational force parameters have been determined. The positive sign of $A_{2}$ determined for Halley indicates that its nucleus rotates in a direct sense, the same as the orbital motion.

Yeomans and Kiang (1981) began their investigation into Halley's past motion with an orbit based on the 1607,1682 , and 1759 observations, and then numerically integrated the comet's motion back to 1404 BC. Planetary and nongravitational perturbations were included at each integration step. In nine cases, the perihelion passage 
times calculated by Kiang (1971) from Chinese observations were redetermined and the unusually accurately observed perihelion times in 141,374, and $837 \mathrm{AD}$ were used to constrain the computed motion of the comet. The dynamic model, including terms for the nongravitational effects, successfully represented all the existing Chinese observations, assuming constant values for the nongravitational parameters $A_{1}$ and $A_{2}$. Thus it seems that the spin axis has remained stable, without substantial precessing, for more than two millenia, as has the comet's ability to outgas. This latter result is consistent with a nearly constant intrinsic brightness over roughly the same interval (Broughton, 1979). From the list of orbital elements given by Yeomans and Kiang (1981) from $1404 \mathrm{BC}$ to $1910 \mathrm{AD}$, one can make a crude estimate of Halley's minimum dynamic age. The heliocentric distance of the descending node decreased from $1.74 \mathrm{AU}$ in $1404 \mathrm{BC}$ to $0.85 \mathrm{AU}$ in $1910 \mathrm{AD}$. If this rate were constant into the distant past then the comet would have crossed the ecliptic plane near Jupiter's orbit in 14300 BC. If Jupiter happened to be nearby during such a nodal crossing, then perhaps comet Halley was captured into its current orbit configuration at such an occasion. Hence, in 1986, comet Halley will have been in an Earth-crossing orbit for at least 16000 years and probably much longer.

\subsection{RECENT ORBITAL WORK ON COMET HALLEY}

The recovery of comet $P /$ Halley on 1982 October 16 using the $5 \mathrm{~m}$ Hale reflector on Mt. Palomar showed the comet's image only 9 arc sec away from the ephemeris position predicted by Yeomans (Jewitt et al., 1982). At this writing additional accurate astrometric positions have been recorded at 19 different observatories. Recovered at a distance of more than $11 \mathrm{AU}$ from the Sun, the comet showed no obvious activity and the initial observational accuracy is not limited by the uncertainty of the comet's center of mass within an extensive coma. The initial astrometric positions of comet Halley are generally accurate to within 1 arc sec with a series of 25 positions from La Silla (Chile) in late January 1984 achieving a heretofore unrealizable RMS accuracy of less than 0.5 arc sec in both right ascension and declination.

There are also efforts underway to improve the accuracy of the older data. Morley (1983) has used the SAO Star Catalog to improve the positions taken at Cordoba (Argentina) during the last apparition, Klare et al. (1984) have remeasured some of the 1909-1911 Heidelberg (Germany) plates, and Bowell (1982) has remeasured some of the 1910-1911 Lowell Observatory (Arizona) plates that were never used for astrometric positions before. Pereyra and West (1984) have remeasured approximately 70 plates taken at Cordoba (Argentina) in 1910, and Roser (1984) has re-reduced much of the 1835-1836 visual data using modern reference star catalogs.

Within the Astrometry Net of the IHW, the computer software for cometary orbit determination has been improved somewhat. Incoming observation times in universal time (UTC) are reduced to ephemeris time (ET), the observatory's coordinates are assigned, and the right ascension and declination are corrected for the small effects of ecliptic aberration. Once verified and weighted the observations are stored in reverse chronological order on the master data file for use by the orbit determination program. 
This latter program takes into account the comet's nongravitational perturbations, as well as the planetary perturbations, at each time step. The local error allowed at each time step can be input and the time steps of the numerical integration adjusted to limit the local error to the input tolerance. The partial derivatives of the observables are numerically integrated along with the comet's equations of motion. To be consistent with the reference frames used by the various flight projects to comets Halley and Giacobini-Zinner, the comet's equations of motion also include general relativistic effects by means of the parametrized space-time metric of the Eddington-Robertson-Schiff formalism. Currently this program uses a batch-processed, weighted least squares technique for the analysis. The program can store and use a priori information matrices and map covariance matrices to specified epochs. For example, the improved orbit determination program was used to establish a prediction for the 1986 perihelion passage time based on a new fit to the data from the 1759,1835 , and 1910 returns. If this program had been available prior to the comet's recovery, the predicted time of perihelion passage would have been 1986 February 9.486. At this writing the most recent orbit for Halley is based on 597 observations over the interval from 1835 August 21 to 1985 April 11, as represented by solution 1 in Table II. This table includes perihelion times for six apparitions, with very small differences among the several solutions. For example, the 1986 time of perihelion passage, as predicted using solution 2, is 1986 February 9.4859 , whereas the best value, using solution 1, is February 9.4507 . If there were a significant time dependence in the nongravitational acceleration coefficients, this

TABLE II

Orbit solutions and elements for the motion of $P /$ Halley

\begin{tabular}{|c|c|c|c|c|}
\hline Solution & 1 & 2 & 3 & 4 \\
\hline Observation interval & $1835-1985$ & $1759-1911$ & $1682-1836$ & $1607-1759$ \\
\hline Number of observations & 597 & 718 & 278 & 125 \\
\hline RMS residual (arc sec) & 2.27 & 12.1 & 23.5 & 77.0 \\
\hline Weighted RMS residual & 1.59 & 2.51 & 5.4 & 34.1 \\
\hline \multirow[t]{2}{*}{$A_{1}\left(10^{-8} \mathrm{AU}_{\mathrm{day}}{ }^{-2}\right)$} & 0.1437 & 0.1083 & -0.0081 & \\
\hline & \pm 0.0107 & \pm 0.0217 & \pm 0.1087 & \\
\hline \multirow[t]{2}{*}{$A_{1}\left(10^{-8} \mathrm{AU} \mathrm{day}^{-2}\right)$} & +0.015508 & +0.015683 & +0.015324 & \\
\hline & \pm 0.000004 & \pm 0.000011 & \pm 0.000017 & \\
\hline \multirow{2}{*}{\multicolumn{2}{|c|}{$\begin{array}{r}\text { Perihelion } 1607 \text { Oct. } \\
1682 \text { Sept. }\end{array}$}} & & 27.5478 & 27.5451 \\
\hline & & 15.3689 & 15.2794 & 15.2951 \\
\hline 1759 Mar. & 13.0299 & 13.0623 & 13.0623 & 13.0620 \\
\hline 1835 Nov. & 16.4397 & 16.4396 & 16.4396 & \\
\hline 1910 May & 20.1786 & 20.1785 & 20.1120 & \\
\hline 1986 Feb. & 9.45069 & 9.4859 & & \\
\hline 1986 Feb. & 19.0 & & & \\
\hline Perihelion, $q$ (AU) & 0.587997 & & & \\
\hline Eccentricity, $e$ & 0.9672726 & & & \\
\hline Inclination, $i$ (deg) & 162.23931 & & & \\
\hline Long. asc. node $\Omega$ (deg) & 58.14398 & & & \\
\hline Arg. perihelion $\omega(\mathrm{deg})$ & 111.84677 & & & \\
\hline
\end{tabular}


close agreement would not be maintained. Values for the nongravitational parameters $A_{1}$ and $A_{2}$ corresponding to three of these solutions (shown in Table II) confirm that not only is $A_{2}$ (the tangential coefficient, which has the larger effect on the time of perihelion passage) essentially constant with time, but also that it is much better determined than $A_{1}$.

The concluding rows of Table II specify the elements corresponding to solution 1. Here the angular quantities are referred to the ecliptic plane and the mean equinox of 1950.0, and times are shown in ET. At this writing this set of elements provides the smallest residuals among hundreds of observations linking three or more apparitions.

Alternate nongravitational force models have been tried in an effort to improve on the existing model (Marsden et al., 1973). Gas production rates computed by Divine and Newburn (1983) were evaluated at each integration step using a comet-centered rocket-like thrust direction as denoted by Sekanina (1981a). Thus this new model allowed for comet outgassing at rate tied to the visual light curve (cf. Section 3), and was asymmetric with respect to perihelion. In addition, the thermal lag angle, inclination $I$ and argument $\Phi$ of the rotational pole (cf. Section 4.3) were variable parameters in the model testing procedure. The attempted solutions proved to be insensitive to input values of $\Phi$ and although the final solutions were not completely satisfactory, the optimum values for $I$ and the lag angle were 30 and $5 \mathrm{deg}$, respectively. No combination of the input variable values could noticeably decrease the residuals from those of the prior model. It seems likely that additional improvements in the solutions using this alternate nongravitational force model will have to await information on the gas production and spin axis orientation to be measured in early 1986.

\section{Overview of Cometary Activity}

For short-period comets, quantitative analyses of observations of gas emission bands and of the dust continuum with modern instrumentation yield values for gas and dust production rates which permit prediction of that comet's activity for subsequent perihelion passages. Because such data are unavailable for $P / \mathrm{Halley}$, an alternate prediction technique is required. The following sections describe a photometric analysis through which Halley's activity can be crudely predicted from visual observations in prior apparitions.

\subsection{THE SEMI-EMPIRICAL PHOTOMETRIC THEORY}

In 1910, the time of Halley's most recent perihelion passage, cometary astronomy was rather primitive technologically and theoretically. Quantitative knowledge of Halley was limited to the mechanics of its motion, and this knowledge was inaccurate because the effects of the reactive force of escaping gases (the nongravitational force, cf. Section 2) were unknown and not included. The predicted time of perihelion passage was off by three days. Observations of Halley were limited to visual and photographic techniques except for an attempt by Stebbins (1910) to use a primitive photocell (photomultipliers would not be invented for nearly three decades). The only truly quantitative work was 
a beautiful study of tail brightness by Schwarzschild and Kron (1911) that was far in advance of its time (Newburn and Yeomans, 1982). With this limited data base, Halley models have had to be built largely by analogy with other comets for which more recent, quantitative observations exist.

Known comets display a vast range of brightnesses. Even short period comets range from Halley at about absolute magnitude 3 to objects such as Haneda-Campos at 13 or 14, a factor of at least 10000 in intrinsic brightness, while new comets can be far brighter than Halley. Therefore, a comet model must be tied to some actual measure of that comet's brightness. The only measure available even today for the majority of short period comets is a magnitude determined either visually or photographically. The visual magnitudes are far more common and often more reliable for any large, diffuse object brighter than about 12 th magnitude. The observer derives a visual magnitude by comparing the comet (either in-focus or out-of-focus, depending on the technique) with an out-of-focus star of comparable, known brightness (Morris, 1980). Ideally the comparison star should be of roughly the same spectral type as the Sun. A graph of the visual magnitude as a function of time or of heliocentric distance is called the visual light curve. Its pre-perihelion and post-perihelion branches may differ, as they do for Halley.

The spectral response of the dark adapted human eye (scotopic vision) is quite different from that for bright light (photopic vision; e.g., Alpern, 1978). Superposition of the curve of scotopic spectral sensitivity on any cometary spectrum (except very rare $\mathrm{CO}_{2}$ dominated objects) shows that more than $95 \%$ of the light perceived by the eye comes from two sources, the Swan bands of $\mathrm{C}_{2}$ and the continuum (Newburn, 1983). The relative radiance of the five principal Swan bands, fluorescing in sunlight, is a known function only of the heliocentric distance. The continuum is made up of components of sunlight scattered from the cometary nucleus and from the multitude of dust particles in the cometary coma, the latter completely dominating except at very large heliocentric distance. The central question, then, is what relation the $\mathrm{C}_{2}$ and dust, which make up the visible light, have to overall cometary activity. Is the relation sufficiently unique to permit reasonable models to be constructed from the visible light curve? Newburn $(1979,1981)$ explored the relation in some detail, and the answer is affirmative, with certain exceptions and limitations which are more obvious now that more comets have been studied than in 1981.

Newburn (1981) developed a simple photometric equation in which the sum of contributions from nucleus, gas and dust comprised the total observed luminance in the scotopic passband as measured by the visible light curve, in the form

$$
p_{N} R_{N}^{2} \frac{\Phi_{N}}{\Phi_{\pi}}+B Q_{\mathrm{H}}^{2}+C Q_{H} \Phi_{s}=r^{2} \times 10^{0.4\left(m_{\odot}-m_{1}+5 \log \Delta\right)} \text {. }
$$

Here the conditions of observation include heliocentric distance $r$ (units km), geocentric distance $\Delta(\mathrm{AU})$, and phase angle, and the observations yield cometary apparent visual magnitude $m_{1}$, for comparison with that of the Sun $m_{\odot}$ (where $m_{\odot}=-26.8$ at $r=1 \mathrm{AU})$. The nucleus is described by its radius $R_{N}(\mathrm{~km})$, geometric albedo $p_{N}$, and 
phase function $\Phi_{N} / \Phi_{\pi}$, and the dust (cf. Section 7) by its scattering function $\Phi_{s}$, where both $\Phi_{N}$ and $\Phi_{s}$ depend on phase angle (cf. Section 9). Both the coefficients $B\left(\mathrm{~km}^{2} \mathrm{~s}^{2}\right)$ and $C\left(\mathrm{~km}^{2} \mathrm{~s}\right)$, and the derived hydrogen production $Q_{\mathrm{H}}\left(\mathrm{s}^{-1}\right)$, are functions of $r$.

When a comet is inactive (i.e., $Q_{\mathrm{H}}=0$ ), Equation (3.1) reduces to the expression for reflection from asteroid surfaces. When a comet is active $\left(Q_{\mathbf{H}}>0\right)$ the terms proportional to $B$ and $C$ represent the effective cross sections for gas (i.e., $\mathrm{C}_{2}$ ) emission and dust scattering, respectively.

It seems logical to assume that, at least for a given comet, the dust production term would be linear in $Q_{\mathrm{H}}$, while recognizing that the mixing ratio of dust to gas probably would vary from comet to comet. Recently it has been found that this is not always true. Newburn and Spinrad (1985) showed that the actual dust loading of gas in the coma of $P /$ Encke decreases enormously as the comet approches the Sun. Sekanina and Larson (1984) found that the mass loading in Halley's jets was 10 to 100 times that for the surrounding coma. Since Halley's spin axis has an obliquity near $30^{\circ}$ (cf. Section 4.3), the mass loading of its coma undoubtedly changes too. As a nominal model for Halley we retain linear mass loading for the general background, but expect that local areas of much higher loading will occur (cf. Section 8).

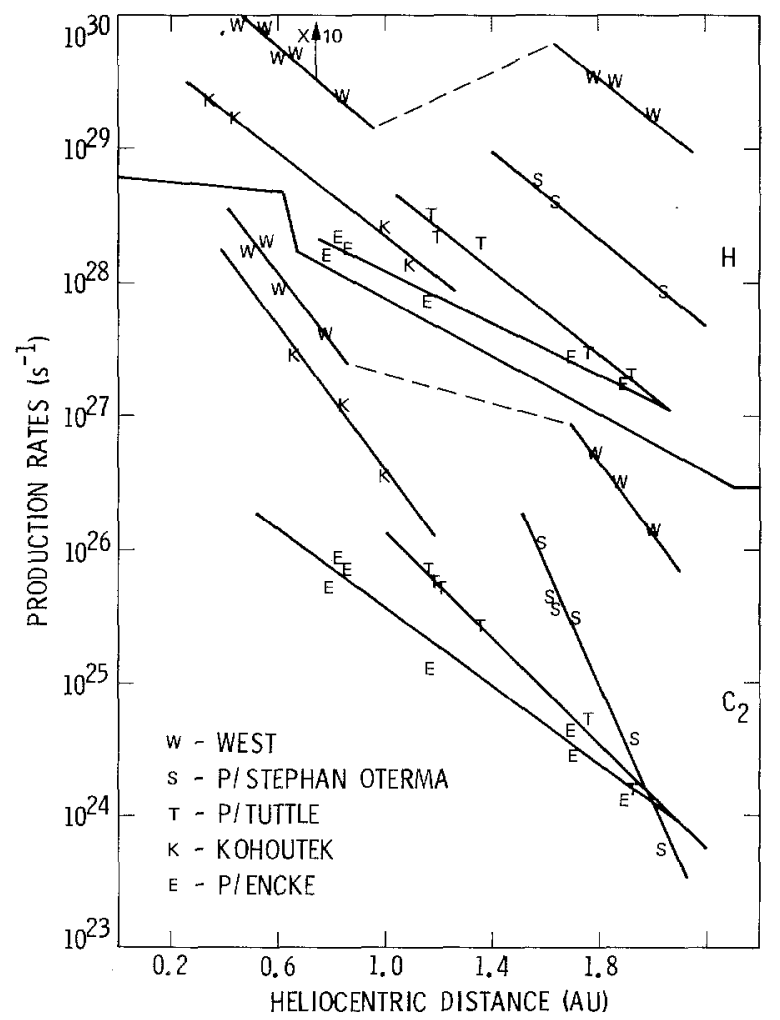

Fig. 1. Production rates of $\mathrm{H}$ and $\mathrm{C}_{2}$ for five comets using data from Newburn and Spinrad (1984), A'Hearn and Cowan (1975), A'Hearn et al. (1977), Feldman et al. (1974), Opal et al. (1974), and Drake et al. (1976). Values for $H\left(C_{2}\right)$ appear above (below) the irregular separator line connecting the ordinate scales. 
If $\mathrm{C}_{2}$ were formed only as the simple photodissociation product of a well mixed parent molecule, the gaseous emission term should also be linear in $Q_{\mathrm{H}}$. In studies for the earliest version of the photometric theory, Newburn (1979) could not fit existing data with a linear expression but could readily do so with a quadratic one. Figure 1 incudes $\mathrm{H}$ and $\mathrm{C}_{2}$ production rates for five comets; where measurements were not directly available they were derived from [OI] or $\mathrm{CN}$ data. Note that in every case the slope of the $\mathrm{C}_{2}$ line is steeper than that of the $\mathrm{H}$ line (there is some distortion, since a semi-log plot was used to spread the points out more in heliocentric distance). In some cases the best exponent for $Q_{\mathrm{H}}$ in the second term of Equation (3.1) would be greater than two (e.g., $P /$ Stephan-Oterma) and in other cases less (e.g., $P /$ Tuttle), but in no case studied could it be as small as one. When such data are unavailable, as for Halley, it is best to stay with two.

The coefficient in the gas emission term should be proportional to the local lifetime of the $\mathrm{C}_{2}$ molecule, so we next introduce its lifetime $\tau_{\mathrm{C}_{2}}=1.2 \times 10^{5} \mathrm{~s}$ at $r=r_{0}=1 \mathrm{AU}$ in the form

$$
B=\mathscr{R} \tau_{\mathrm{C}_{2}}\left(r / r_{0}\right)^{2} .
$$

Here the resonance fluorescence efficiency $\mathscr{R}\left(\mathrm{km}^{2} \mathrm{~s}\right)$ has been evaluated using both magnitude and production rate observations for several comets by Newburn (1981,

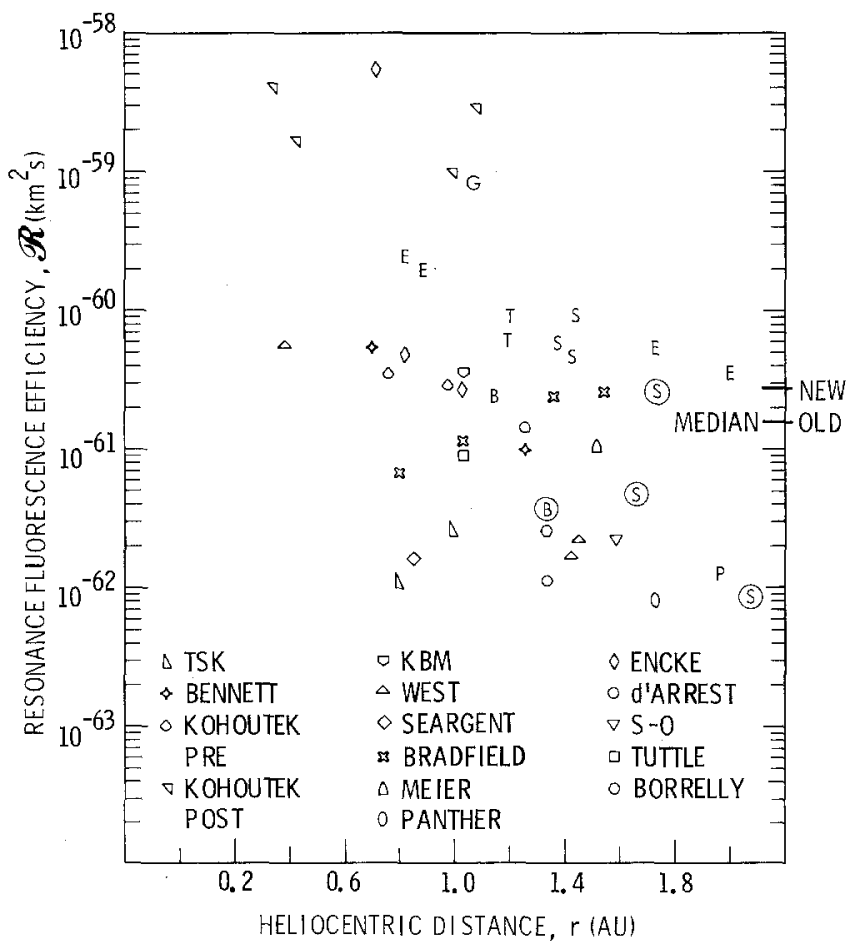

Fig. 2. Values for resonance fluorescence efficiency, computed from data for several comets (from Newburn, 1984). 
1984), with the median results that $\mathscr{R}=4 \times 10^{-61} \mathrm{~km}^{2} \mathrm{~s}$ for short period comets, and $\mathscr{R}=2 \times 10^{-61} \mathrm{~km}^{2} \mathrm{~s}$ for intermediate and long period comets. In this evaluation it is found that, as expected, $\mathscr{R}$ is roughly independent of $r$, even though it displays a broad range $\left(10^{-62}\right.$ to $\left.5 \times 10^{-59} \mathrm{~km}^{2} \mathrm{~s}\right)$ among the comets studied, as shown in Figure 2.

An additional feature of the theory and its calibration is the ratio of the light scattered by dust to that from gas in the scotopic passband, in the form

$$
\delta=\frac{C Q_{\mathrm{H}} \Phi_{s}}{B Q_{\mathbf{H}}^{2}}=\delta_{1}\left(r / r_{0}\right)^{n_{1}} \frac{\Phi_{s}}{\Phi_{\perp}} .
$$

Some justification for this form is given by Newburn (1981), values for the dust scattering function $\Phi_{s}$ (and $\Phi_{\perp}$ at $\theta_{s}=90^{\circ}$ ) have been used as given by Divine (1981b; cf. Figure 20), and the parameter values derived from observation are $\delta_{1}=0.416, n_{1}=2$ for $r<1.25 \mathrm{AU}$, and $\delta_{1}=0.333, n_{1}=3$ for $r>1.25 \mathrm{AU}$ (Newburn, 1981).

With these modifications, then, Equation (3.1) takes the form

$$
p_{N} R_{N}^{2} \frac{\Phi_{N}}{\Phi_{\pi}}+(1+\delta) B Q_{\mathbf{H}}^{2}=r^{2} \times 10^{0.4\left(m_{\odot}-m_{1}+5 \log \Delta\right)}
$$

and its solution for the hydrogen production $Q_{\mathrm{H}}$ is

$$
Q_{\mathrm{H}}=\left[\frac{r_{0}^{2} \times 10^{0.4\left(m_{\Theta}-m_{1}+5 \log \Delta\right)}-p_{N} R_{N}^{2} \Phi_{N} / \Phi_{\pi}}{(1+\delta) \mathscr{R} \tau_{\mathrm{C}_{2}}}\right]^{1 / 2} .
$$

For Halley at $r<2$ AU the quantity $p_{N} R_{N}{ }^{2}$ is negligible, and, using the smaller of the above values for $\mathscr{R}$, we can derive $Q_{\mathbf{H}}$ from the visual light curve.

\subsection{PREDiCTIONS FOR HALLEY IN 1986}

Although there were numerous observations of the visual magnitude of Halley in 1910, they were made by many different observers using different instruments and techniques. A fundamental problem is that Halley was very close to Earth and low on the horizon in 1910 when it had the same heliocentric distance at which spacecraft will encounter it in 1986. The visible coma then covered four times the solid angle of the moon, so no comparison star could be thrown that far out of focus. Being low on the horizon, where there was considerable atmospheric absorption, it was difficult to find a suitable comparison star at the same elevation. The most recent studies (Marcus, 1983; Bortle and Morris, 1984) suggest that Halley's visual magnitude, post-perihelion at $r=\Delta=1 \mathrm{AU}$, has not changed significantly from about 3.1 over the past 20 centuries. Recent post-perihelion light curves for Halley are shown in Figure 3 (Newburn, 1984). We recommend using the Bortle and Morris curve, which is described by linear segments of the form

$$
m_{1}-5 \log \Delta=H_{\mathrm{O}}+2.5 n_{\mathrm{o}} \log r,
$$

for $r$ and $\Delta$ in AU, using values for $H_{0}$ and $n_{0}$ given in Table XIV (Appendix A). For $r>2$ AU the quadratic relation between $\mathrm{C}_{2}$ and $\mathrm{H}$ production fails, but between 


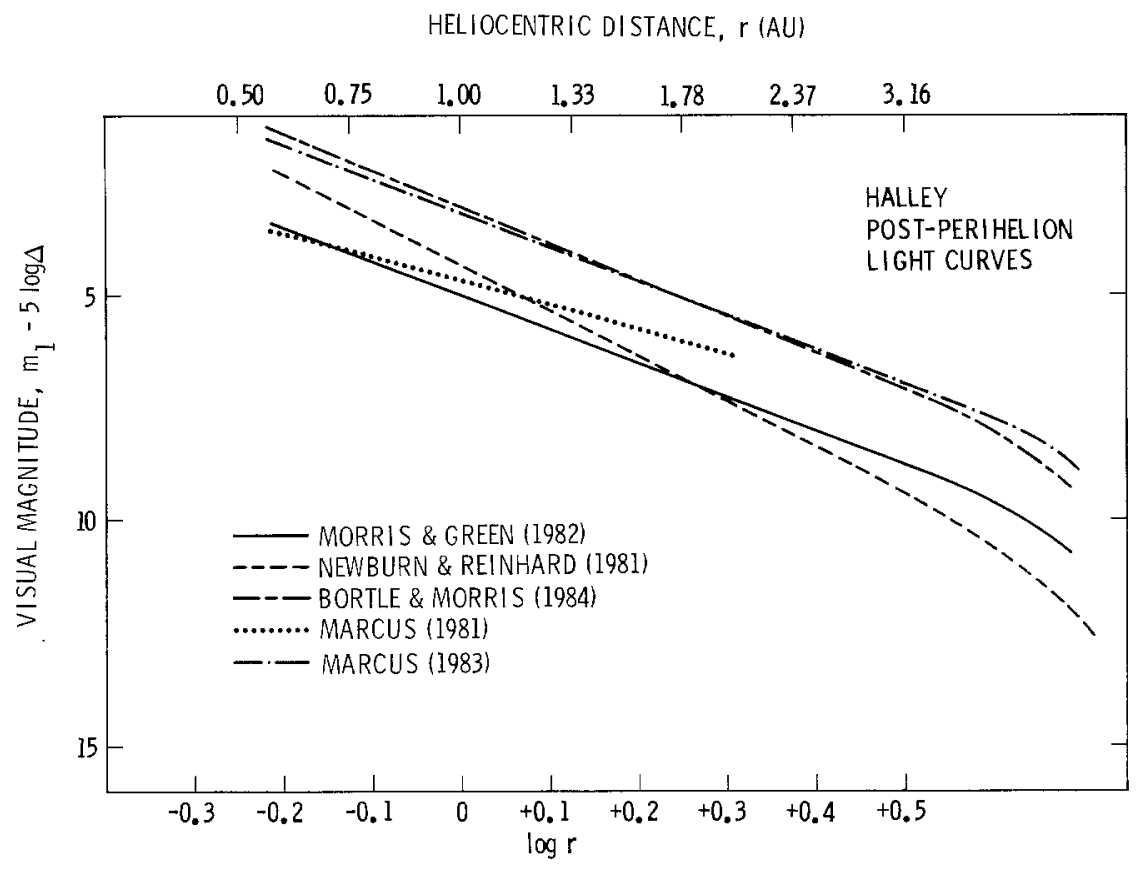

Fig. 3. Postperihelion light curves for $P /$ Halley.

perihelion and $2 \mathrm{AU}$ postperihelion it should work well, if Halley is a normal comet of its intrinsic brightness.

Most things we know about Halley indicate that it is quite normal. Spectra taken in 1910 reveal no unusual line strengths, recorded on typical uncalibrated blue sensitive plates (cf. Figure 6.1). Certainly there was no abnormal amount of $\mathrm{CO}^{+}$such as dominated the spectra of Comet Morehouse 1908 III and Humason 1962 VIII, which may have been $\mathrm{CO}_{2}$-controlled. The apparent strength of the continuum was very average as compared to the emission features. Halley seems to be a normal comet for its size and magnitude, although the brightest of the 100 or so known periodic objects. Halley was active at 3.5 AU pre-perihelion in 1909 and remained active out to $5.2 \mathrm{AU}$ postperihelion in 1911, which is unusual. If Halley is strictly water controlled, this would be possible only if an area remained continually in sunlight or if the rotation were exceptionally slow.

Models have usually been calculated by assuming there is 10 to $20 \%$ by number of molecules other than water. Water can hold about one foreign molecule in its frozen lattice for every six water molecules, forming a clathrate whose sublimation properties remain essentially the same as for pure water ice (Delsemme and Miller, 1971). Molecules of species more volatile than water would be lost quickly (and at large heliocentric distance) unless trapped within the clathrate or at some distance below the surface. Using $F_{T W}$ as the ratio of total molecules to water by number, and with two $\mathrm{H}$ atoms released per molecule of water, we obtain the total gas production $Q_{g}$ 
(molecule $\mathrm{s}^{-1}$ ) in the form

$$
Q_{g}=\frac{F_{T W}}{2}\left(\frac{r_{0}^{2} \times 10^{0.4 m_{\odot}}}{\mathscr{R} \tau_{\mathrm{C}_{2}}}\right)^{1 / 2}\left[\frac{10^{-0.4 H_{0}-n_{0} \log \left(r / r_{0}\right)}}{(1+\delta)}\right]^{1 / 2},
$$

from Equation (3.5) (neglecting the term proportional to $p_{N}$ ). Using the value $F_{T W}=1.2$, and the magnitude parameters specified in Table XIV (for the results of Bortle and Morris, 1984; as in Figure 3), we obtain values for $Q_{g}$ throughout an apparition of $P /$ Halley as shown in Table V (Section 5).

Lastly, observations at visible and infrared wavelengths have each been used independently to estimate the dust production in comets, but the results in the two approaches are inversely proportional to the dust albedo or to the dust residence time in the field of view, respectively, and effective values for these latter quantities are not well determined from the observations used. Considering Comet Bennett (1970 II) as a reasonable analog for Halley (based on general similarities in appearance and spectra), Newburn (1979) adopted $\mu=0.5$ for the ratio of dust to gas production by mass, taken from the dust dynamical analysis by Sekanina and Miller (1973), who used a geometric albedo $p=0.1$ for the dust. A more recent analysis of the dust continuum in the visible (Newburn and Spinrad, 1984) yields $\mu$ in the range 0.004 to 0.764 for several comets, among which 0.1 to 0.3 includes about half the entries. The alternate (infrared) approach has been applied to several comets by various authors, among whom Ney (1982) obtains values which imply $\mu=0.2$ for Bennett. Somewhat arbitrarily, this latter value has been adopted for the current, homogeneous models of $P /$ Halley; it would not be surprising to find an additional 0.2 in the dust jets (cf. Section 8).

\section{Parameters for the Nucleus}

Because the cometary nucleus is so inaccessible to direct observations, most descriptions rely on indirect inferences which are consistent with the diverse cometary phenomena discussed elsewhere in this review. The development of quantitative results for Halley's nucleus is even less secure, proceeding from meagre data and analogy with other comets, as presented in the following sections.

\subsection{COMPOSITION, STRUCTURE, AND DENSITY}

As originated by Whipple $(1950,1951)$, the icy conglomerate nucleus is a satisfactory source for all observed cometary phenomena (cf. Section 1). In this model the nucleus is a single, solid object containing both ices and mineral grains, i.e., volatile and refractory components, as thoroughly reviewed by Delsemme (1982), Donn and Rahe (1982), and Mendis et al. (1985).

The volatile component consists primarily of simple molecules of abundant elements, namely $\mathrm{H}_{2} \mathrm{O}, \mathrm{NH}_{3}, \mathrm{CO}_{2}$, and other likely parents of observed species (Table VII). As shown by Biermann et al. (1982), Yamamoto et al. (1983), and others, it is possible to start with interstellar abundance ratios for the elements involved, model the thermodynamic and chemical processes in which the elements form simple compounds and 
freeze into an icy matrix (here of course the excess hydrogen and helium are not captured), and produce a mixture which is consistent with molecular abundances observed in cometary comae after sublimation, collision and photolysis (Section 6). In the sublimation process, ices more volatile than water can be released from the surface relatively far from the Sun (thus accounting for cometary activity even beyond $3 \mathrm{AU}$ ), so that in the inner solar system the activity is controlled by the sublimation of water ice (cf. Section 5.1). Thus the volatile component of the outermost layers is largely water ice, with some other molecules trapped in hydrate clathrate. It seems likely that below such surface layers the ice composition more nearly reflects the interstellar abundance ratios of $\mathrm{C}, \mathrm{N}$, and $\mathrm{O}$, and that more complex molecules (e.g., organics, parents for $\mathrm{C}_{2}$ and $\mathrm{S}_{2}$, etc.) may be present also. Similar results follow if comets originated beyond Saturn in the primordial solar nebula (Yamamoto, 1985).

The refractory component contains the minerals which constitute cometary dust, as discussed in Section 7.3. Here, as expected, the most abundant elements are typically $\mathrm{C}, \mathrm{O}, \mathrm{S}, \mathrm{Al}, \mathrm{Si}, \mathrm{Ca}, \mathrm{Mg}$, and Fe; Table X (Section 7) lists several of the minerals (many uncommon on Earth because of differences in formation conditions) which comprise carbonaceous chondrites as one likely sample of nonvolatile material from cometary nuclei. Greenberg $(1982,1983)$ and others suggest that conditions and abundances in interstellar space may well be appropriate for the formation and inclusion of such grains in icy cometary nuclei, although this has not been confirmed (for more discussion see Section 7.3).

There is considerable evidence that the nucleus is chemically undifferentiated (Delsemme, 1982; Donn and Rahe, 1982), with the likely exception of near-surface layers (Houpis et al., 1985, and Section 5). In this case dust and ice would be well-mixed throughout. If dust grains in the porous, ice-free mantle are easily separated from one another by the action of the outflowing gas (as suggested by the theory cited in Section 5.1), then whatever cohesion and strength exists in the nucleus may be provided primarily by the icy component acting as a glue (rather than by the crystal structure of the refractory component). Observations of nucleus splitting (Sekanina, 1982) and dissipation (Sekanina, 1984a) suggest that the cohesion is rather weak, possibly because the nucleus' structure is poorly cemented or not compact. Such weakness is to be expected considering likely formation processes (e.g., Donn et al., 1985) and thermal cycling (e.g., Kührt, 1984), especially since the latter may involve phase transitions from amorphous to crystalline ice, with the associated volume change and mechanical stress (e.g., Patashnick et al., 1974; Klinger, 1980); it is also consistent with the dust descriptions in Section 7.3. A mixture of water ice, at mean density $0.9 \mathrm{~g} \mathrm{~cm}^{-3}$, with dust grains, at mean density $3.0 \mathrm{~g} \mathrm{~cm}^{-3}$ (for the individual units), in the ratio 0.2 (dust to ice, by mass; cf. Section 7 and Table XV) leads to a density $1.02 \mathrm{~g} \mathrm{~cm}^{-3}$ for the nucleus. We thus choose the round value $1.0 \mathrm{~g} \mathrm{~cm}^{-3}$ as a typical estimate of the nucleus' bulk density, as consistent with the range of values in the literature (e.g., $0.5 \mathrm{~g} \mathrm{~cm}^{-3}$, Wallis and McPherson, 1981; and $2 \mathrm{~g} \mathrm{~cm}^{-3}$, Delsemme and Yeomans, 1984).

As in clear from the processes discussed in Chapters 5 and 8 , the surface layers of cometary nuclei may well be unique in the solar system. Structural inhomogeneities 


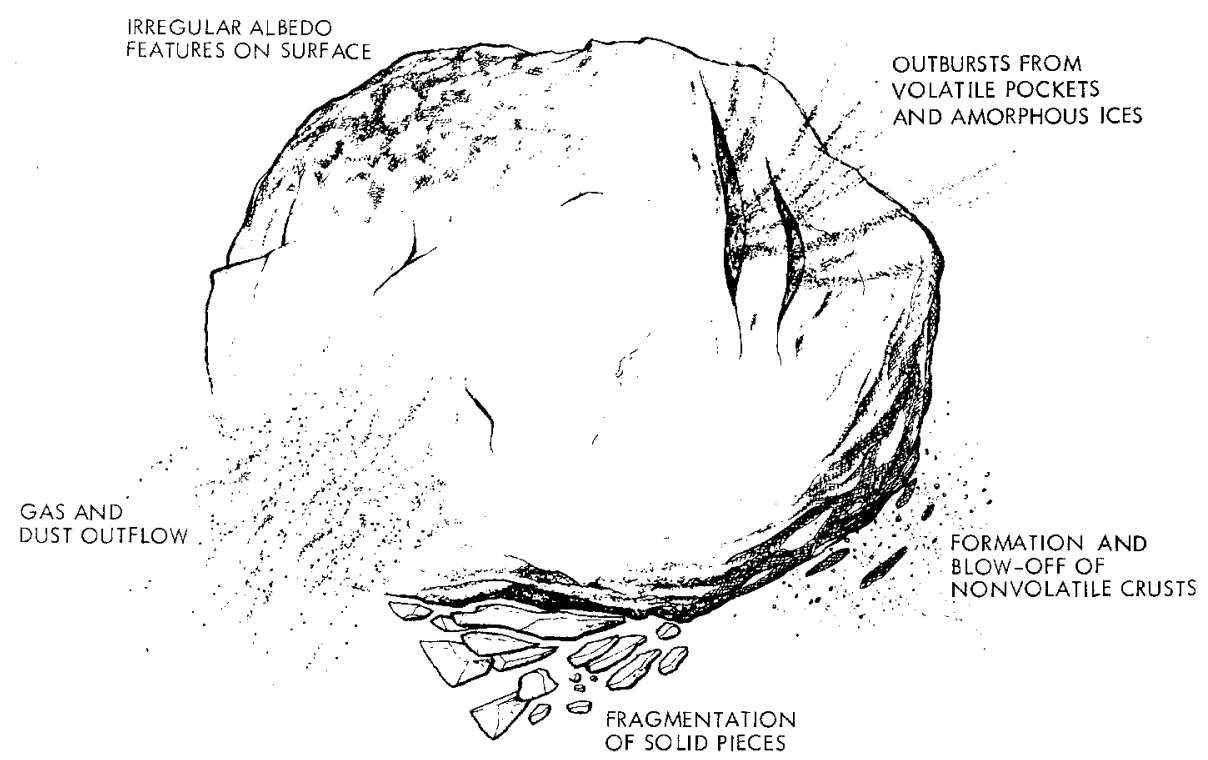

Fig. 4. Sketch of an imaginary cometary nucleus, illustrating its possible irregular shape and several inhomogeneous and time-variable processes which may occur on its surface (from Weissman and Kieffer, 1981).

originally present in the interior will result in variegated activity when they become exposed at the surface, and could be responsible for observed changes in cometary brightness and dust features. Weissman and Kieffer (1981) provide a brief, interesting list of potential surface features, as illustrated in Figure 4. The irregular shape shown there is consistent with those derived from models of rotational precession and nongravitational forces, among which Giacobini-Zinner provides an extreme example (diameter ratio 8.3, Sekanina, 1985a).

\subsection{Albedo, SIZE, AND MASS}

Large ranges of uncertainty in quantitative values for physical parameters of cometary nuclei are endemic even in modern studies of recent comets. This is so because the nucleus is very difficult or impossible to observe from Earth. It is even possible that data from the Halley flyby spacecraft will not much improve this situation, especially if the nucleus' visibility is poor (cf. Section 9.4).

At large heliocentric distances beyond 2 or $3 \mathrm{AU}$ it is sometimes possible to make cometary observations in which the coma makes no obvious contribution (i.e., gaseous emission lines absent from spectra, extended comae absent from direct photographs or visual images, and/or irregular variations absent from photometric magnitudes). This would be consistent with the very low vapor pressure of pure water ice at expected equilibrium temperatures far from the Sun. If other volatile species are also inactive (perhaps by virtue of being covered by a mantle, as in Section 5.1), and if there is no material orbiting the nucleus, the photometric equation (3.1) might be used with $Q_{\mathrm{H}}=0$ to obtain an area-albedo product (or $p_{N} R_{N}^{2}$ ) from an observed magnitude, just as for 
TABLE III

Determinations of sizes for cometary nuclei. The symbols in column 3 indicate that the following kinds of measurements were included in the analysis: $P$, photometry at large heliocentric distance; $N$, nongravitational acceleration; $R$, radar detection; $S$, spin axis precession; $L$, latent heat and/or rate of sublimation; $V$, velocity distribution of emitted dust. In some cases ranges, or values for polar and equatorial radii, are specified.

\begin{tabular}{|c|c|c|c|}
\hline Comet & $\begin{array}{l}\text { Radius } \\
R_{N}(\mathrm{~km})\end{array}$ & $\begin{array}{l}\text { Processes } \\
\text { considered }\end{array}$ & Reference \\
\hline Arend-Rigaux & $4.4,5.1$ & $I$ & Tokunaga and Hanner (1985) \\
\hline IRAS-Araki-Alcock & $5 \pm 0.6$ & $I$ & Hanner et al. (1985a) \\
\hline IRAS-Araki-Alcock & 3 to 4 & $R$ & Goldstein et al. (1984) \\
\hline$P /$ Grigg-Skjellerup & $>0.4$ & $R$ & Kamoun et al. (1982b) \\
\hline$P /$ Encke & $1.5_{-1.0}^{+2.3}$ & $R$ & Kamoun et al. (1982a) \\
\hline$P /$ Encke & inconclusive & $P, L)$ & \\
\hline Tago-Sato-Kosaka & $2.2 \pm 0.3$ & $P, L\}$ & Delsemme and Rud (1973) \\
\hline Bennett & $3.8 \pm 0.5$ & $P, L)$ & \\
\hline Bennett & 2.6 & $V$ & Sekanina and Miller (1973) \\
\hline$P /$ Kopff & $1.2,1.45$ & $N, S$ & Sekanina (1984b) \\
\hline$P /$ Giacobini-Zinner & $0.15,1.25$ & $N, S$ & Sekanina (1985a) \\
\hline$P /$ Comas Solá & $0.5,1.1$ & $N, S$ & Sekanina $(1985 c)$ \\
\hline$P /$ Schwassmann-Wachmann 1 & $40 \pm 5$ & $P, I$ & Cruikshank and Brown (1983) \\
\hline
\end{tabular}

asteroids. In a few exceptional cases an $r^{-2}$ dependence for the observed brightness suggests that the nucleus has been seen directly (e.g., Arend-Rigaux and $P /$ Neujmin 1 ; Sekanina, 1976). More commonly residual activity, low albedo, small diameter, ephemeris uncertainty, and lack of application of really powerful instrumentation conspire to make this approach unproductive. Some of these factors also mitigate against the successful application to comets of occultation techniques which have provided good asteroidal diameters. Table III lists some of the cases in which a photometric technique (label $P$ in column 3 ) has led to quantitative results. The table also lists several other determinations for the sizes of cometary nuclei using a variety of data sources. In spite of some surprisingly clever approaches (see column 3), no one technique has provided reliable, narrowly bounded results for more than a few comets, and in many cases the errors are large (not shown for every row in the table). However, the general range of radii of the nuclei of active comets has been well established, with a middle range 1 to $5 \mathrm{~km}$ (excepting Schwassmann-Wachmann 1).

For Halley the early models used an analogy with Bennett (see its radius determinations in Table III) to suggest a radius near $3.0 \mathrm{~km}$ (Whipple, 1981; Newburn and Reinhard, 1981). Subsequently, by considering both the nongravitational forces and the magnitude limit established by a failed recovery attempt in 1981, Belton and Butcher (1982) tentatively recommended a geometric albedo $p_{N}=0.17$ and radius $R_{N}=2 \mathrm{~km}$ for Halley, although the limits set by their analysis included a radius $R_{N}=3 \mathrm{~km}$ comfortably as well. In an exceptional effort, use of a CCD detector on the 5-m Hale telescope enabled the 1982 recovery observations of Halley at the record heliocentric distance $r=11.2 \mathrm{AU}$. As reported by Jewitt and Danielson (1984), the combination of 
these data with later observations using identical instrumentation is consistent with an upper limit $2.2 \pm 0.2 \mathrm{~km}^{2}$ for the product $p_{N} R_{N}^{2}$. Nevertheless, even in this work irregular variations of about one magnitude on time scales $\lesssim 1$ day suggest that sporadic activity (and/or rotation combined with shape or albedo inhomogeneities; cf. Section 4.3) may contribute to the photometry (which is here being stretched to the limit of available technology). As tentatively suggested by these authors (Jewitt and Danielson, 1984), the values $p_{N}=0.1$ and $3<R_{N}<5 \mathrm{~km}$ could represent an acceptable interpretation (in agreement with the results $\left(R_{N}=3.1_{-1.6}^{+3.4} \mathrm{~km}\right)$ of Sicardy et al. (1983). Delsemme and Yeomans (1984) derived a mass $M_{N}=(2$ to 13$) \times 10^{13} \mathrm{~kg}$ for Halley (from the increase in the nongravitational parameter $A_{2}$ ) which is consistent with the radii cited above, although the recent estimate by Hughes (1985) is somewhat larger $\left(M_{N}=2.2 \times 10^{14} \mathrm{~kg}\right)$.

Thus it is not surprising that present models for Halley use parameter values for the nucleus which lack thorough, quantitative justification, even though they are consistent with the results cited here and elsewhere. These values, as we best estimate them in mid-1985, are radius $R_{N}=3000 \mathrm{~m}$, density $\rho_{N}=1.0 \mathrm{~g} \mathrm{~cm}^{-3}$, and geometric albedo $p_{N}=0.06$ (at a wavelength $0.5 \mu \mathrm{m}$ ). This albedo is only slightly larger than that for cometary dust (Section 7.1), because even a small admixture of dark grains can drastically lower the albedo of icy particles. If the nucleus surface intensity is isotropic and uniform on the sunlit hemisphere, the corresponding Bond albedo is $A_{N}=0.12$. The analysis discussed below (Section 4.3) does not suggest a highly irregular shape, so the simplest assumption of a spherical, homogeneous nucleus is used for modeling purposes. Thus we use

$$
M_{N}=\frac{4 \pi}{3} \rho_{N} R_{N}^{3}
$$

to obtain the mass $M_{N}=1.13 \times 10^{14} \mathrm{~kg}$ from the above values.

\subsection{ROTATION PERIOD AND AXIS}

Although Bessel (1836a) was the first to investigate the periodicity of oscillatory motions of Comet Halley's ejecta he believed he had observed (deriving a period 4.6 days), serious attempts to determine this comet's rotation period began only several years ago. Whereas the techniques vary, the basic idea of at least two types of study is identification of recurring events.

Whipple $(1980,1982)$ applied his zero-date method to coma-diameter measurements reported by various observers in the apparitions $1835 / 36$ and 1910 to derive a rotation period of $10^{\mathrm{h}} 19^{\mathrm{m}}$. His approach is based on the assumption of uniformly expanding halos at a rate of one halo per rotation from a single active area, and therefore the true rotation period could be an integer multiple of Whipple's value.

Analysis of Halley's brightness variations at large heliocentric distances has been pursued by several researchers. However, attempts to derive a rotation period from the magnitudes obtained between recovery in 1982 and early 1984 have been unsuccessful. Although short-term variations with an amplitude of 1 mag or more were clearly 
recorded, West and Pedersen (1984) have found no apparent periodicity in the normalized light curve and attributed the effects to irregular, transient events of unspecified origin. Sekanina (1985b) agreed, pointing out that his power-spectrum analysis revealed, among others, solutions that coincide with the Sun's rotation period and its submultiples. He speculated that the short-term surges in brightness could be brought about by the expulsion of fine dust from isolated regions on the comet's surface, charged electrostatically by the solar wind and solar UV radiation impinging unobstructed on the nucleus. Such a scenario was proposed for distant comets by Mendis et al. (1981), who concluded that the conditions on the dark side of the nucleus should be strongly modulated by the local solar-wind field. Although short-term changes in the solar wind would be essentially random, weak correlations with the solar rotation and/or the sector-structure pattern of the interplanetary magnetic field (as revealed by moderately significant peaks in the light curve's power spectrum) would be expected (Sekanina, 1985b) because of the existence of high-speed compression regions, whose leading edges usually coincide with the magnetic sector boundaries. Jewitt and Danielson (1984) concluded from the time scale of the fluctuations that they were not due to transient ejection of refractory grains. However, their conclusion applies only to uniformly expanding halos of nonvolatile particles whose velocities follow Bobrovnikoff's (1954) law. LeFevre et al. (1984), Lecacheux and LeFevre (1984), and Morbey (1985), on the other hand, seem more optimistic about the prospects of the rotation-period determination from Halley nucleus photometry, although they also have problems with the multitude of possible solutions.

Larson and Sekanina (1984) set a lower limit near one day for Halley's rotation period from the degree of curvature of spiral jets seen on digitally processed 1910 photographs. Their approach was based on the concept of evolution of the spiral jets into expanding halos, interpreted as the product of continuous dust ejection from discrete sources on the sunlit hemisphere of a rotating nucleus (Sekanina and Larson, 1983; cf. Section 8). The quantitative modeling of the evolving jets resulted first in the adoption of a tentative rotation period of $1.73 \pm 0.4$ days (Sekanina and Larson, 1984). Further refinement of the spin vector, based on a considerably augmented sample of jets in 1910 (Larson and Sekanina, 1985), has subsequently resulted in an improved value of 2.17 days (Sekanina and Larson, 1986), which is equivalent to almost exactly five times Whipple's rotation period.

Sekanina and Larson's (1984) model was also employed to determine the direction of Halley's rotation axis. Figure 5 defines the two angles involved: obliquity $I$, which is the angle subtended by the spin axis and by the normal to the orbit plane; and argument $\Phi$, which is the angle between the comet's 'vernal equinox' and the direction to the Sun at perihelion. The obliquity is less than or greater than $90^{\circ}$ when the rotation is, respectively, prograde or retrograde with respect to the orbital motion. For more detail, see Sekanina (1981a, b).

Two important limitations on the position of Halley's rotation axis are readily inferred from the comet's orbital motion: (1) according to Whipple's (1950) icy-conglomerate model, the sense of the observed secular deceleration (Yeomans, 1977) indicates that 


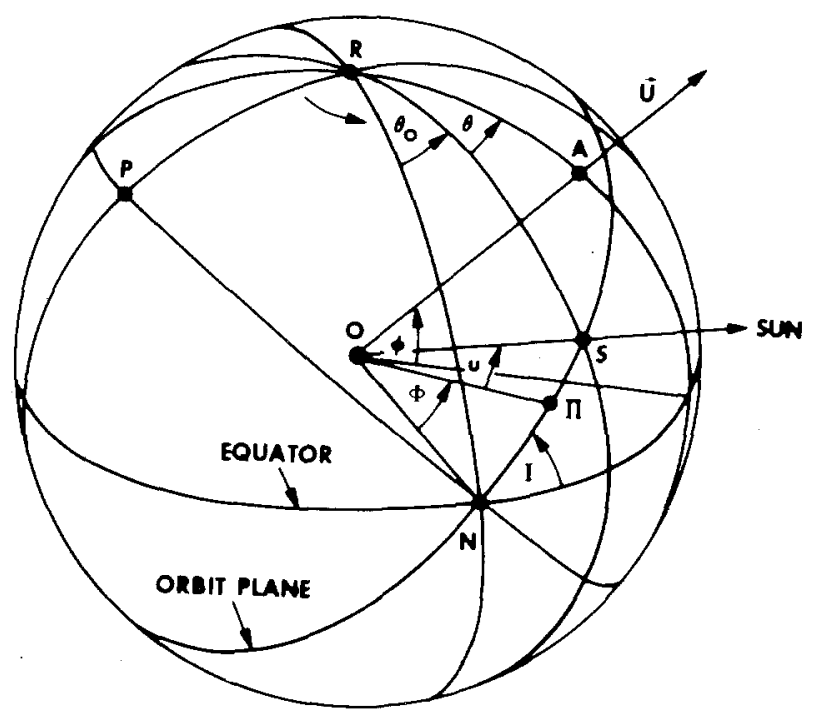

Fig. 5. Orientation on a spherical rotating cometary nucleus: $O$ is its center, $P$ the northern orbital pole, $R$ the northern pole of rotation (with the arrow indicating the sense of rotation), $N$ the ascending node of the orbit plane on the equator (the comet's vernal equinox), $\Pi$ the subsolar point at perihelion, and $S$ the subsolar point at true anomaly $u$. Angle $I$ is the obliquity of the orbit plane to the equator and $\Phi$ is the argument of the subsolar meridian at perihelion. The angles $\theta_{0}$ and $\theta$ are measured positive east between the meridians containing the vernal equinox $N$, the subsolar point $S$, and an active area $A$ at latitude $\phi$, from which dust ejection occurs in the radial direction $\mathbf{U}$ (from Sekanina and Larson, 1984; see discussion in Section 8.3).

the nucleus rotation is prograde (Newburn and Yeomans, 1982); and (2) the virtual invariability of the transverse component of the non-gravitational force (directly responsible for the deceleration; cf. Section 2.1) over an interval of at least two millennia further implies the absence of measurable precession of the spin axis (Yeomans and Kiang, 1981) and, together with a fairly well-defined radial component of the force, suggests that the axis does not lie near the orbit plane.

Although very helpful in constraining the obliquity $I$, the orbital evidence provides no limits on the argument $\Phi$. Fortunately, the concept of discrete spiral jets (Section 8) proves a powerful tool in setting stringent bounds on $\Phi$. The basic idea is obvious: the observed sense of unwinding of a jet from the nucleus condensation depends on the angle between the position of the spin axis and the direction to Earth. If a jet is observed to unwind counterclockwise, the Earth is located in the general direction of the comet's north pole (to which, by definition, the spin vector points), and vice versa. Jets observed at various times allow one to combine a number of these conditions and find a common range of consistent solutions. Particularly restrictive constraints apply to comets with rapidly changing geometry relative to Earth during close approaches. Fortunately Halley approached Earth within 0.2 AU both in 1835 and in 1910.

Application of the various constraints and, in particular, the successful fitting of a large number of dust features, each detected on at least three consecutive dates, have 
TABLE IV

Parameter values for the rotation of Halley's nucleus (from Sekanina and Larson, 1986)

\begin{tabular}{lr}
\hline Rotation axis (equinox 1950.0) & \\
Argument of subsolar meridian at perihelion, $\Phi$ & $300^{\circ}$ \\
Obliquity of orbit plane to equatorial plane, $I$ & $30^{\circ}$ \\
Ecliptic longitude of north pole & $333^{\circ}$ \\
Ecliptic latitude of north pole & $-42^{\circ}$ \\
Right ascension of north pole & $357^{\circ}$ \\
Declination of north pole & $-49^{\circ}$ \\
Rotation period & \\
Sidereal, $P$ & $52.1 \mathrm{hr}$ \\
Synodic at perihelion & $53.1 \mathrm{hr}$ \\
\hline
\end{tabular}

led to a well-defined position of Halley's rotation axis. Its north celestial pole is in the constellation Phoenix. Table IV provides three alternate specifications for the direction of Halley's rotation axis, as well as the best available value of the period (Sekanina and Larson, 1986).

\section{Nucleus-Coma Interface Region}

Details of the processes close to the nucleus surface in which the sublimating ice/gas molecules escape into interplanetary space and carry along the released dust grains are described in the following sections.

\subsection{SURFACE TEMPERATURES AND GAS PRODUCTION}

Following Delsemme and Swings (1952), elementary steady-state kinetic theory was widely used to relate the gas mass flux $Z$ (sample units $\mathrm{kg} \mathrm{m}^{-2} \mathrm{~s}^{-1}$ ) to the nucleus' surface temperature $T_{N}(\mathrm{~K})$. This approach yields the classical result that

$$
Z=P_{s}\left(M_{g} / 2 \pi k T_{N}\right)^{1 / 2} .
$$

With the ratio $\gamma$ of specific heats, Boltzmann's constant $k=1.38 \times 10^{-23} \mathrm{~J} \mathrm{~K}^{-1}$, the mean molecular mass $M_{g}(\mathrm{~kg})$, latent heat $L\left(\mathrm{~J} \mathrm{~kg}^{-1}\right)$ of vaporization from the solid, and the vapor pressure $P_{r}\left(\mathrm{~N} \mathrm{~m}^{-2}\right)$ at a reference temperature $T_{r}(\mathrm{~K})$ for the sublimating ice and gas, the sublimation presure $P_{s}\left(\mathrm{~N} \mathrm{~m}^{-2}\right)$ and speed of sound $w_{s}\left(\mathrm{~m} \mathrm{~s}^{-1}\right)$ are given by

$$
P_{s}=P_{r} \exp \left[\frac{M_{g} L}{k}\left(\frac{1}{T_{r}}-\frac{1}{T_{s}}\right)\right]
$$

and

$$
w_{s}=\left(\gamma k T_{s} / M_{g}\right)^{1 / 2} \text {. }
$$


Equation (5.1) assumes an outflow speed equal to $w_{s}$, although other values (differing by as much as a factor $2^{1 / 2}$ ) have been used. The solar energy available for the sublimation of water ice and the extreme sensitivity of Equations (5.1) and (5.2) to variations in $T_{s}$ have the general result that comets have surface temperatures of the order of $200 \mathrm{~K}$ when they have heliocentric distance $r$ near $1 \mathrm{AU}$.

Clearly, however, for an outflow velocity comparable to the speed of sound, steady state kinetic theory is a poor approximation. In addition, as pointed out by Wallis (1982) and Mendis et al. (1985), the mean free path of gas molecules near the surface is so large (order of $10 \mathrm{~cm}$ ) that a hydrodynamic approach is required for an improved calculation of $Z$. The 'mantle/reservoir' model of Gombosi et al. (1985) yields a hydrodynamic estimate for the gas production rate. In this model the mantle is porous dust which contains a reservoir of stationary gas. At every point the gas and dust share a common temperature $T$ which increases from the sublimating temperature $T_{s}$ at depth $y=\Delta$ (the interface between the mantle and the core of solid ice and dust) to the temperature $T_{N}$ at the outer surface $(y=0)$. As described by Horanyi et al. (1984), this temperature variation is governed by the steady-state energy equation

$$
\frac{\mathrm{d}}{\mathrm{d} y}\left(\kappa \frac{\mathrm{d} T}{\mathrm{~d} y}\right)=-\frac{3 k Z}{2 M_{g}} \frac{\mathrm{d} T}{\mathrm{~d} y} .
$$

Here the thermal conductivity $\kappa\left(\mathrm{W} \mathrm{K}^{-1} \mathrm{~m}^{-1}\right)$ includes both contact and radiation terms. Note that in Equation (5.4) the divergence of the energy flux is non-zero, since we assume that the penetrating vapor is in thermal equilibrium with the dust throughout the mantle and so represents a sink of heat.

To solve Equation (5.4) the model imposes the boundary condition at $y=\Delta$ that the downward heat flux be used exclusively for sublimation, requires that $P=P_{s}$ (cf. Equation (5.2)) and $Z$ each be constant throughout the stationary gas reservoir contained in the mantle, and imposes the boundary condition at $y=0$ given by

$$
J_{a}=\varepsilon_{N} \sigma T_{N}^{4}+\frac{3 Z}{2 M_{g}} k\left(T_{N}-T_{s}\right)+L Z .
$$

Here $J_{a}$ is the radiation flux absorbed at the surface $\left(\mathrm{W} \mathrm{m}^{-2}\right.$; it may contain contributions both from direct sunlight and from the dust scattered and thermal radiation), $\varepsilon_{N}$ is the infrared emissivity of the nucleus, and $\sigma$ is the Stefan-Boltzmann constant $\left(2 \pi^{5} k^{4} / 15 c^{2} h^{3}\right)$.

Finally the mantle/reservoir model of Gombosi et al. (1985) uses the result from gas dynamics texts (e.g., Zucrow and Hoffmann, 1976) that the gas mass flux (at $y=0$ ) from a spherically-symmetric stationary reservoir to a low pressure external medium can be approximated as

$$
Z=\frac{P_{s}}{w_{0}}\left(\frac{1+\cos \alpha}{2}\right) \gamma\left(\frac{2}{\gamma+1}\right)^{(\gamma+1) / 2(\gamma-1)} .
$$

Here $w_{0}$ is the sound speed evaluated at the surface $\left(T=T_{N}\right.$; cf. Equation (5.3)), and 
the effective half opening angle $\alpha$ for the discharging diverging flow is of the order of $90^{\circ}$, as determined by Gombosi et al. (1985) using the time-dependent numerical solutions of the full dusty hydrodynamic problem (cf. Section 5.2). Their calculations show that the steady-state kinetic theory model (Equation (5.1)) overestimates the gas mass flux $Z$.

If the porous mantle in such a model were not subject to erosion by the escaping gas, its thickness would increase with time as the sublimating ice/dust core recedes below the surface. In this case the gas production would be quenched and the surface temperature would satisfy Equation (5.5) for $Z \rightarrow 0$. As first modeled by Mendis and Brin (1977), time-variable erosion of the mantle competes with the thickness increase produced by the shrinking core so as to provide a possible explanation for the brightness hysteresis of comets during single and.successive perihelion passages. Horanyi et al. (1984) provided a quantitative formulation of these processes in their 'friable sponge' model, containing three basic assumptions:

(a) Dust grains in the mantle have the same spatial configuration as in the core;

(b) Destruction of the mantle takes place only on the surface, where the large, immobile particles break into smaller pieces before their accumulation violates assumption (a); and

(c) The mass loss rate of the mantle is proportional to the momentum flux of the outflowing gas, with a proportionality factor that is constant for each comet.

Using the friable sponge model Horanyi et al. (1984) determined the relation between sublimating and surface temperatures for various mantle thicknesses. By introducing a new parameter, representing the 'friability' of cometary dust grains, they calculated the temporal evolution of the mantle thickness during several revolutions and found that comets behave differently depending on their friability: a small friability parameter means gradual dust accumulation that finally quenches the comet's activity, whereas large friability precludes the development of any significant mantle.

On the basis of the friable sponge model Houpis et al. (1985) have developed a chemically differentiated multilayer model. They assume that the nucleus originally consisted of an ice/dust conglomerate (cf. Section 4.1) where various volatile ices are mixed with water ice in a ratio too large for all the volatile molecules to be trapped in clathrate lattice. As such a nucleus approaches the Sun, all the untrapped volatiles escape first. However, as the upper layers of volatiles sublimate, they leave behind a steadily growing mantle of evacuated clathrate ice. Consequently, the volatile species first diffuse out through the clathrate mantle before escaping into space. More importantly this upper mantle partially insulates the more volatile core. This calculation explains the high activity of new comets at large heliocentric distance and predicts that all evolved comets will eventually behave as if they were water dominated, independent of the original chemical composition deep inside the core. Additional features of mantle development for short period comets, as influenced by the effects of rotation, are discussed in detail by Fanale and Salvail (1984).

Figure 6 exemplifies the variation of $T_{N}, T_{s}$, and $Z$ as a function of mantle thickness $\Delta$, using values from Horanyi et al. (1984), for an isolated water-ice nucleus at $r_{0}=1 \mathrm{AU}$ 

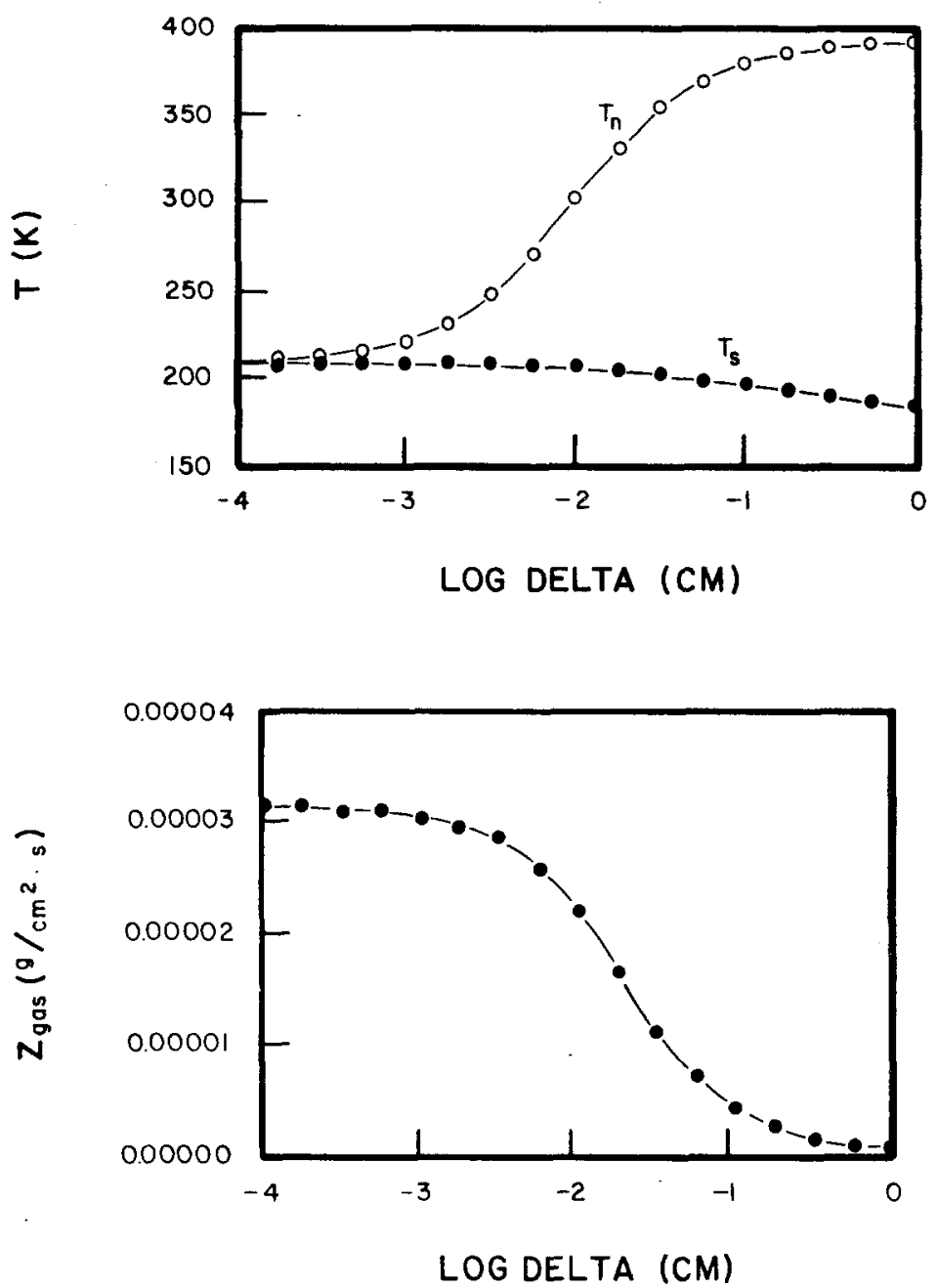

Fig. 6. Dependence on mantle thickness of surface and sublimating temperatures (upper panel) and of gas mass flux (lower panel) for sample comet nucleus parameters from Horanyi et al. (1984; cf. their Figure 3).

from the Sun $\left(J_{a}=L_{\odot} / 4 r_{0}^{2} \pi=1350 \mathrm{~W} \mathrm{~m}^{-2}\right)$, including the modification of Equations (5.2) and (5.6) for a non-zero value of the dust-to-ice ratio by mass in the core. Note that as the mantle approaches a thickness of about $1 \mathrm{~cm}$ the gas mass flux $Z$ drops several orders of magnitude, while the surface temperature rises to its limiting value.

Infrared observations of comet IRAS-Araki-Alcock (1983d) published by Hanner et al. (1985a) indicate that at a heliocentric distance near $1 \mathrm{AU}$ this comet had a very small production rate and high surface temperature. Numerical comparisons with models based on the above analysis suggest that a mantle thickness about $0.5 \mathrm{~cm}$, a subsolar surface temperature near $T_{N}=400 \mathrm{~K}$, and a sublimating temperature $T_{s}=190 \mathrm{~K}$ may be reasonable values for this comet. The observations are in good 
agreement with the prediction for a low friability comet, where surface dust accumulation largely quenches the gas and dust production with the result that the comet's behavior approaches that of an inactive, asteroidal object.

\subsection{GAS-DUST INTERACTION AND TERMINAL VELOCITIES}

It was recognized early that gas outtlow plays an important role in the cometary dust production process (Orlov, 1935). In early treatments of the gas-dust interaction it was assumed that the drag coefficient was independent of the gas flow parameters and that the gas velocity was constant in the dust acceleration region (Whipple, 1951; Dobrovolsky, 1966). This latter assumption was dropped when Shul'man (1969) solved a simplified set of gas dynamical equations and obtained an accelerating gas flow.

A two-component treatment of the gas-dust interaction was published almost simultaneously by Probstein (1969), Brunner and Michel (1968), and Shul'man (1969) assuming that the heavy dust grains have no thermal motion and collide only with gas molecules. Using a free molecular drag approximation, Probstein (1969) obtained a drag coefficient which was a slowly varying function of relative Mach number. Assuming perfect thermal accommodation between gas molecules and dust grains he also derived a dust-gas heat transfer function. Probstein (1969) also found a transonic solution to the coupled dust and gas equations using a single characteristic grain size. His solution represented a major step forward since he substituted for the traditional gas energy conservation equation a combined gas-dust energy integral. However, it was later pointed out by Shul'man (1972) and Hellmich (1981) that Probstein's energy conservation equation is not valid if the dust is heated by solar radiation. The radiative transfer problem in a dusty cometary coma is far from simple (cf. Equation (9.16)) and has been investigated by a number of groups using methods with various degrees of sophistication (Shul'man, 1972; Hellmich and Keller, 1980; Weissman and Kieffer, 1981; and Marconi and Mendis, 1983).

The steady-state gas equations can be combined into one first order differential equation in the presence of strong gas-dust coupling. This equation has a zero/zero singularity at the sonic point. To obtain a physical transonic solution one has to 'prescribe' the smooth behavior at the critical point. This requires a time-consuming 'shooting' method to force the gas velocity function smoothly through the sonic point (Probstein, 1969; Hellmich, 1981; Gombosi et al., 1983; and Marconi and Mendis, 1985). On the other hand a time-dependent treatment of the gas-dust interaction process does not yield singular differential equations; instead the transonic solution evolves naturally with time (Gombosi et al., 1985).

In this section we summarize the time-dependent equations which govern the gas and dust interaction in a spherically symmetric inner coma. With cometocentric distance $R$ $(\mathrm{m})$ and time $t(\mathrm{~s})$ as independent variables, the continuity, momentum and energy equations of the neutral gas are

$$
\frac{\partial}{\partial t}\left(A_{f} \rho_{g}\right)+\frac{\partial}{\partial R}\left(A_{f} \rho_{g} u\right)=0
$$




$$
\frac{\partial}{\partial t}\left(A_{f} \rho_{g} u\right)+\frac{\partial}{\partial R}\left(A_{f} \rho_{g} u^{2}+A_{f} P_{g}\right)=P_{g} \frac{\partial A_{f}}{\partial R}-A_{f} F_{g d}
$$

and

$$
\frac{\partial}{\partial t}\left[A_{f} \frac{\rho_{g} u^{2}}{2}+\frac{A_{f} P_{g}}{\gamma-1}\right]+\frac{\partial}{\partial R}\left[A_{f} \frac{\rho_{g} u^{3}}{2}+A_{f} \rho_{g} \frac{\gamma u}{\gamma-1}\right]=A_{f}\left(Q_{x}-Q_{g d}\right)
$$

Here $\rho_{g}, P_{g}$, and $u$ are the local gas mass density $\left(\mathrm{kg} \mathrm{m}^{-3}\right)$, pressure $\left(\mathrm{N} \mathrm{m}^{-2}\right)$, and flow velocity $\left(\mathrm{m} \mathrm{s}^{-1}\right), A_{f}$ is a dimensionless area function, $Q_{x}$ is the external heating rate $\left(\mathrm{W} \mathrm{m}{ }^{-3}\right)$, and $F_{g d}$ and $Q_{g d}$ represent the momentum $\left(\mathrm{N} \mathrm{m}^{-3}\right)$ and energy $\left(\mathrm{W} \mathrm{m}^{-3}\right)$ transfer rates from the gas to the dust. For the dust (neglecting any role for icy grains) we require conservation of particles of every size (i.e., ignoring any possible further fragmentation) to derive the corresponding three equations

$$
\begin{aligned}
& A_{f} \frac{\partial n}{\partial t}+A_{f} V \frac{\partial n}{\partial R}=-A_{f} n \frac{\partial V}{\partial R}-n V \frac{\partial A_{f}}{\partial R}, \\
& \frac{\partial V}{\partial t}+V \frac{\partial V}{\partial R}=P_{g} H_{a},
\end{aligned}
$$

and

$$
\frac{\partial T}{\partial t}+V \frac{\partial T}{\partial R}=P_{g} G_{a} T^{1 / 2}+\frac{3}{a \rho C}\left(\frac{J_{a}}{4}-\varepsilon \sigma T^{4}\right)
$$

Here $n$ represents the local differential concentration $\left(\mathrm{m}^{-4}\right)$ of spherical dust particles with radius $a(\mathrm{~m})$, bulk density $\rho\left(\mathrm{kg} \mathrm{m}^{-3}\right)$, specific heat capacity $C\left(\mathrm{~J} \mathrm{~K}^{-1} \mathrm{~kg}^{-1}\right)$, infrared emissivity $\varepsilon$, temperature $T(\mathrm{~K})$, and radial outflow velocity $V\left(\mathrm{~m} \mathrm{~s}^{-1}\right)$. The absorbed radiative flux $J_{a}\left(\mathrm{~W} \mathrm{~m}^{-2}\right)$ plays the same role as in Equation (5.5), except that for the dust it may vary with position, and the flow accomodation functions $H_{a}$ $\left(\mathrm{m}^{2} \mathrm{~kg}^{-1}\right)$ and $G_{a}\left(\mathrm{~K}^{1 / 2} \mathrm{~s} \mathrm{~m} \mathrm{~kg}{ }^{-1}\right)$ are presented by Probstein (1969) as functions of the local gas temperature $T_{g}$ and relative Mach number

$$
\mathfrak{M}=\cdot(u-V)\left(M_{g} / \gamma k T_{g}\right)^{1 / 2}
$$

Conservation of momentum and energy (Equations (5.8), (5.9), (5.11), and (5.12)) requires that $F_{g d}$ and $Q_{g d}$ be expressed as integrals over products among $G_{a}, H_{a}, \rho, C$, $V$, and $a$ (cf. Equation (3) of Gombosi et al., 1985).

During the last fifteen years several authors solved Equations (5.7) through (5.13) using steady-state approximations with various boundary conditions. These solutions produced transonic gas flows and accelerated dust particles close to the surface of cometary nuclei. Early calculations, using mainly analytic or semi-empirical methods, were summarized by Wallis (1982). Recently more sophisticated numerical models were developed by several groups. Hellmich and Keller (1980), Gombosi et al. (1983), and Marconi and Mendis (1983) published steady-state solutions to Equations (5.7) through 
(5.12) using external dust and gas heating and realistic dust size distributions. The details of these calculations were different, but the general behavior of the solutions agree reasonably well. For a comet like Halley these solutions at $1 \mathrm{AU}$ predict a rapidly cooling, expanding gas flow. At about $R=250 \mathrm{~km}$ the gas temperature is less than about $10 \mathrm{~K}$ and the dust particles have already reached their terminal velocities. At larger cometocentric distance the gas temperature will rise again, but in this region there is no significant gas-dust interaction any more.

To visualize better the gas-dust interaction, Gombosi et al. (1985) introduced an effective area function $A_{e}$ defined by the following differential equation:

$$
\frac{\mathrm{d} A_{e}}{\mathrm{~d} R}=A_{e}\left[\frac{1}{A_{f}} \frac{\partial A_{f}}{\partial R}-\frac{F_{g d}}{P_{g}}+\frac{\gamma-1}{\gamma u P_{g}}\left(Q_{g d}-Q_{x}\right)\right]
$$

Using $A_{e}$, the steady-state forms of the gas equations (5.7) through (5.9) yield the differential equation

$$
\frac{\mathrm{d} u}{\mathrm{~d} R}=-\frac{u}{1-\mathfrak{M}_{g}^{2}}\left[\frac{1}{A_{e}} \frac{\partial A_{e}}{\partial R}\right],
$$

where $\mathfrak{M}_{g}$ is the gas Mach number. Equation (5.15) is just the equation describing the steady, unrestricted discharge of gas into vacuum through a nozzle having an area function represented by $A_{e}$. For unrestricted spherical geometry the gas flows out of the reservoir with the local sonic velocity. The situation changes drastically when the outflowing gas has to drag away dust. In Figure 7 we show the variation of the effective

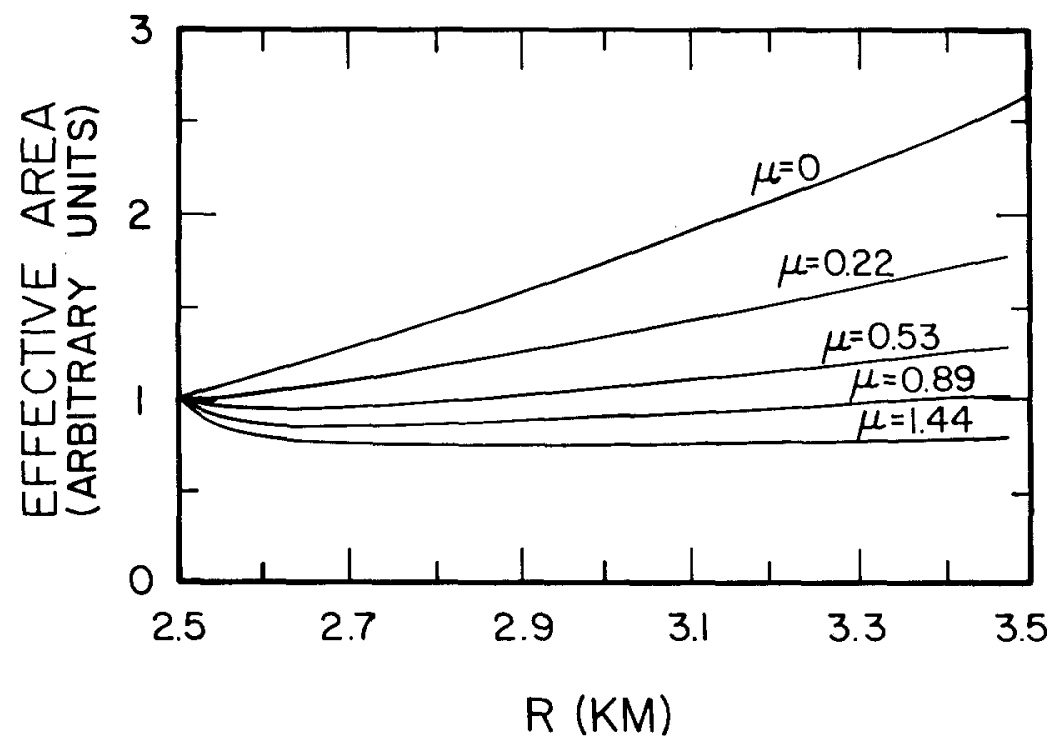

Fig. 7. Variation with cometocentric distance $R$ of the effective area function $A_{e}$, normalized to unity at the surface ( $R=R_{N}=2.5 \mathrm{~km}$ for these sample calculations), and parametrized by the ratio $\mu$ of dust to gas production by mass. 
area function with cometocentric distance $R$. In the calculation $A_{e}$ was normalized to unity at the surface $\left(R=R_{N}\right)$. In this particular case we use one characteristic dust size $(a=0.5 \mu \mathrm{m})$ and identical sublimating and surface temperatures $T_{s}=T_{N}=200 \mathrm{~K}$. The curves are parametrized by the production rate ratio $\mu$ of dust to gas by mass. Figure 7 shows that when there is no dust in the flow $(\mu=0)$ we obtain the textbook case for which $A_{e}$ is proportional to $R^{2}$. For larger values of $\mu, A_{e}$ first decreases, then reaches a minimum value at the sonic point, and then increases. This shows that the dust interaction can in effect be visualized using the concept of a Laval nozzle. First the effective geometry 'narrows' to a throat, and then it 'opens up', allowing the gas to leave the nucleus' surface with subsonic velocity (due to the momentum and energy loss caused by entrained dust), and then go through a sonic point and reach supersonic velocities. This transition would have been impossible in the absence of dust.

One of the most important results of calculations of the dusty gas dynamics in the inner coma is the prediction of dust terminal velocity distributions. Such velocities serve as parameters for dust trajectory calculations which model a wide variety of dust-related cometary features, including dust apex distances and features of the dust coma and tail (cf. Sections 7.4 and 8.2). Calculated dust terminal velocities will also play important roles in targeting the VEGA and GIOTTO spacecraft, as well as in interpreting their dust measurements.

Gombosi et al. (1983) investigated the effect of a realistic dust size distribution on dust terminal velocities. First they solved the coupled dust-gas equation system with a

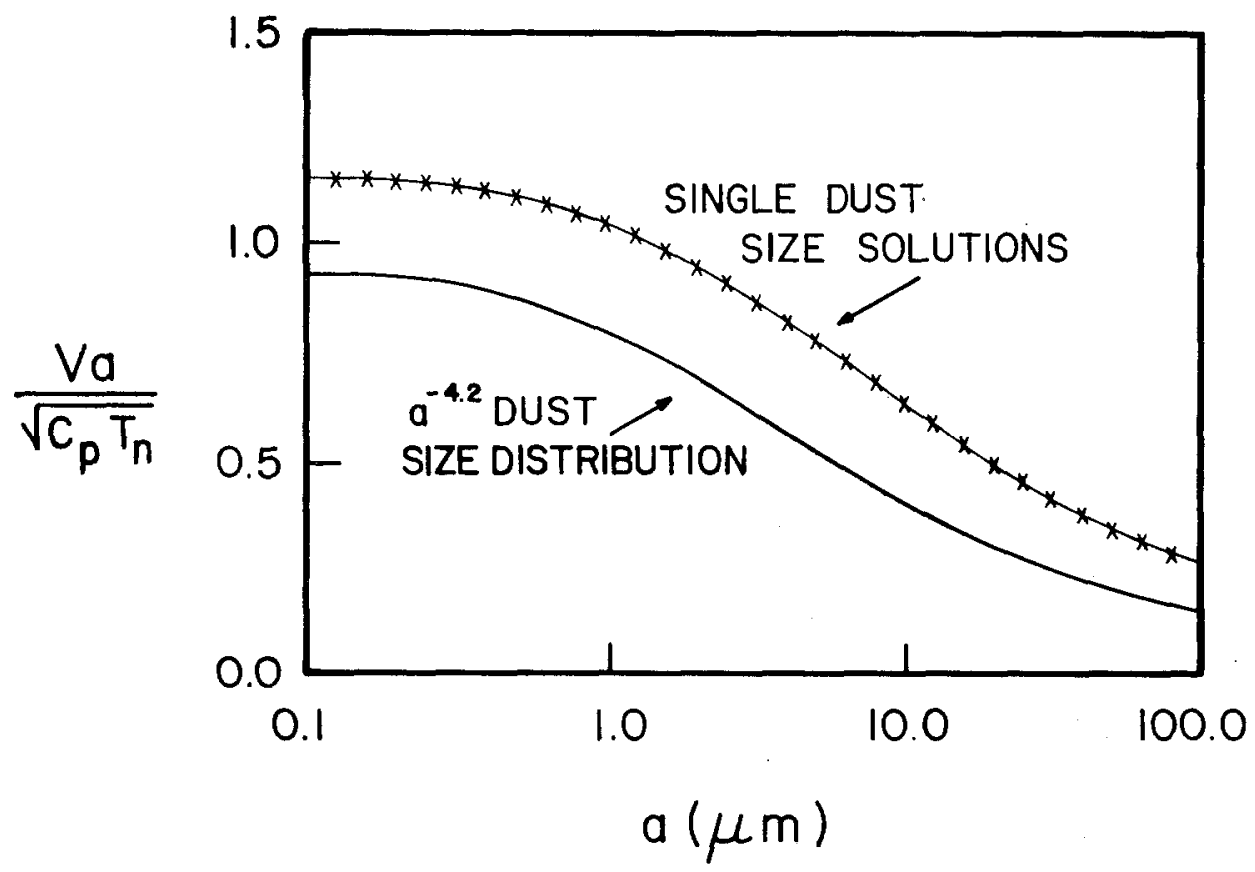

Fig. 8. Dust terminal velocity distributions obtained with one dust size (upper curve) and a realistic dust size distribution (lower curve). Dust velocities are normalized to $\left(C_{p} T_{N}\right)^{1 / 2}$, where $C_{p}$ is the gas specific heat $\left(\mathrm{J} \mathrm{K}^{-1} \mathrm{~kg}^{-1}\right)$ and $T_{N}$ is the surface temperature $(\mathrm{K})$. 
TABLE V

Sample results of steady-state dust-gas hydrodynamic calculations for Halley. Blank lines separate pre-perihelion and postperihelion entries. See text of Section 5.2 for notation and additional explanation.

\begin{tabular}{lllllll}
\hline $\begin{array}{l}\text { Helioncentric } \\
\text { distance }\end{array}$ & $\begin{array}{l}\text { Gas } \\
\text { production }\end{array}$ & $\begin{array}{l}\text { Sublimation } \\
\text { temp. }\end{array}$ & $\begin{array}{l}\text { Gas } \\
\text { terminal }\end{array}$ & & \multicolumn{2}{l}{ Dust term. velocity, $V\left(\mathrm{~m} \mathrm{~s}^{-1}\right)$} \\
\cline { 5 - 6 } & $Q_{g}\left(\mathrm{~s}^{-1}\right)$ & $T_{s}(\mathrm{~K})$ & $\begin{array}{l}\text { velocity } \\
u_{T}\left(\mathrm{~m} \mathrm{~s}^{-1}\right)\end{array}$ & $a=0.4 \mu \mathrm{m}$ & $a=4 \mu \mathrm{m}$ & $a=42 \mu \mathrm{m}$ \\
\hline 2.00 & & & 621 & 135 & 61 & 26 \\
1.50 & $2.3 \times 10^{28}$ & 183 & 631 & 200 & 94 & 41 \\
1.25 & $5.7 \times 10^{28}$ & 188 & 637 & 248 & 121 & 53 \\
1.00 & $9.7 \times 10^{28}$ & 191 & 643 & 307 & 156 & 70 \\
0.75 & $1.7 \times 10^{29}$ & 195 & 653 & 390 & 212 & 99 \\
0.587 & $3.5 \times 10^{29}$ & 200 & 663 & 461 & 268 & 130 \\
0.75 & $6.2 \times 10^{29}$ & 204 & 657 & 490 & 296 & 146 \\
0.88 & $8.4 \times 10^{29}$ & 206 & 652 & 455 & 265 & 128 \\
1.00 & $6.3 \times 10^{29}$ & 204 & 648 & 428 & 242 & 115 \\
1.25 & $5.0 \times 10^{29}$ & 202 & 643 & 377 & 203 & 94 \\
1.50 & $3.3 \times 10^{29}$ & 200 & 639 & 329 & 171 & 77 \\
2.00 & $2.2 \times 10^{29}$ & 197 & 633 & 253 & 124 & 55 \\
\hline
\end{tabular}

realistic dust size distribution function, assuming a spectral index $N=4.2$ (cf. Section 7.2) and using thirty dust size classes. In the next step they solved the same equation system thirty times using single dust sizes, and renormalized the solutions to give a dust terminal velocity distribution. The results are shown in Figure 8, which shows that by using a realistic dust size distribution the dust terminal velocities decrease about $20 \%$.

Gombosi et al. (1985) have published a time-dependent treatment of inner coma dusty hydrodynamics, in which steady-state results are a natural by-product. These authors have also exercised their programs using parameter values for $P / H a l l e y$ developed in other sections of this review (and listed in Appendix A). The resulting dust terminal velocities differ considerably from earlier predictions; sample results for the gas and for three dust sizes are presented in Table $V$ throughout an apparition for Halley. Their complete distributions for terminal velocity are used in the Halley model results which comprise Appendix B, and can also serve as input parameters for large-scale dust calculations or be used directly in interpreting observations.

\section{Cometary Gas}

Reactions of the gas molecules which leave the nucleus' surface with one another and with the solar and local radiation fields modify the flow, temperature, and partition into chemical and ionic species in the coma. The following sections describe these reactions and a Halley gas model. Reasonably thorough discussions of the ions (beyond the scope of this review) are provided by Ip and Axford (1982), Breus (1982), and Mendis et al. (1985). 


\subsection{THE CHEMICAL SPECIES}

At the time of the last apparition of Halley's comet in 1910, cometary spectroscopy was still in its infancy. Even so, several chemical species (both neutral and ionized) were identified in the head and plasma tail. A complete list of these identifications, together with the electronic transitions responsible for their respective emissions, is given in Table VI, while a spectrogram of Halley on 1910 May 27, at a heliocentric distance near $1 \mathrm{AU}$, is shown in Figure 9. Strong emission lines of CN (at wavelength $388 \mathrm{~nm}$ ) and $\mathrm{C}_{2}(474,517$, and $564 \mathrm{~nm})$ are apparent. Detailed quantitative descriptions of the spectral evolution of Halley during its 1910 apparition are given by Bobrovnikoff (1927, 1931). However, since no band emission strengths were measured from the photographic spectra of the comet during that apparition, no quantitative estimates of production rates or abundances of the observed species exist.

Advances in spectrophotometric techniques, together with the extension of the spectral range both shortward (UV) and longward (IR and radio) of optical wavelengths, have resulted in the detection of many more chemical species in subsequent comets. A composite list of all identifications to date is given in Table VII. While the chemically stable molecular species $\left(\mathrm{H}_{2} \mathrm{O}, \mathrm{HCN}, \mathrm{CH}_{3} \mathrm{CN}\right)$ are observed at radio wavelengths, the atomic species $\mathrm{H}, \mathrm{C}, \mathrm{O}$, and $\mathrm{S}$ have been detected via their resonance lines using vacuum UV detectors aboard Earth-orbiting satellites and high-flying rockets. Molecular emissions, among which we include those from many radicals which are highly reactive in solid or collisionally-dominated gas phases, are prominent in the visible portions of cometary spectra. The composite, quantitative emission spectrum for $P /$ Tuttle from 0.1 to $1.02 \mu \mathrm{m}$ wavelength in Figure 10 illustrates the more recent data from many of the chemical species in Table VII.

The emissions that were observed in Comet Halley during its 1910 apparition (Table VI) are among the brightest optical emissions seen in comets. Also, the high degree of spectral uniformity observed in several recent comets, using narrow-band filter photometry (A'Hearn and Millis, 1980; A'Hearn, 1982), leads to the expectation that most, if not all, of the chemical species listed in Table VII will be observed during

TABLE VI

Emission lines identified in photographic spectra of $P /$ Halley in 1910

(cf. Figure 9 and Table XI).

\begin{tabular}{lll}
\hline $\begin{array}{l}\text { Chemical } \\
\text { species }\end{array}$ & $\begin{array}{l}\text { Electronic } \\
\text { transition }\end{array}$ & $\begin{array}{l}\text { Characteristic } \\
\text { wavelength, } \lambda(\mathrm{nm})\end{array}$ \\
\hline $\mathrm{CH}$ & $A^{2} \Delta-X^{2} \Pi_{r}$ & 432 \\
$\mathrm{C}_{2}$ & $d^{3} \Pi_{g}-a^{3} \Pi_{u}$, & $437,474,517,564$ \\
$\mathrm{C}_{3}$ & $\Delta v=2,1,0,-1$ sequences & \\
$\mathrm{CN}$ & $\tilde{A}^{1} \Pi_{u}-\tilde{X}^{1} \Sigma_{g}^{+}$ & 405 \\
& $B^{2} \Sigma^{+}-X^{2} \Sigma^{+}$, & 388,422 \\
$\mathrm{Na}$ & $\Delta v=0,-1$ sequences & 588,589 \\
$\mathrm{CO}^{+}$ & ${ }^{2} P^{0}-{ }^{2} S$ & $401,427,456$ \\
$\mathrm{~N}_{2}^{+}$ & $A^{2} \Pi_{i}-X^{2} \Sigma^{+}$, & 391 \\
\hline & $\Delta v=3,2,1$ sequences & \\
\hline
\end{tabular}



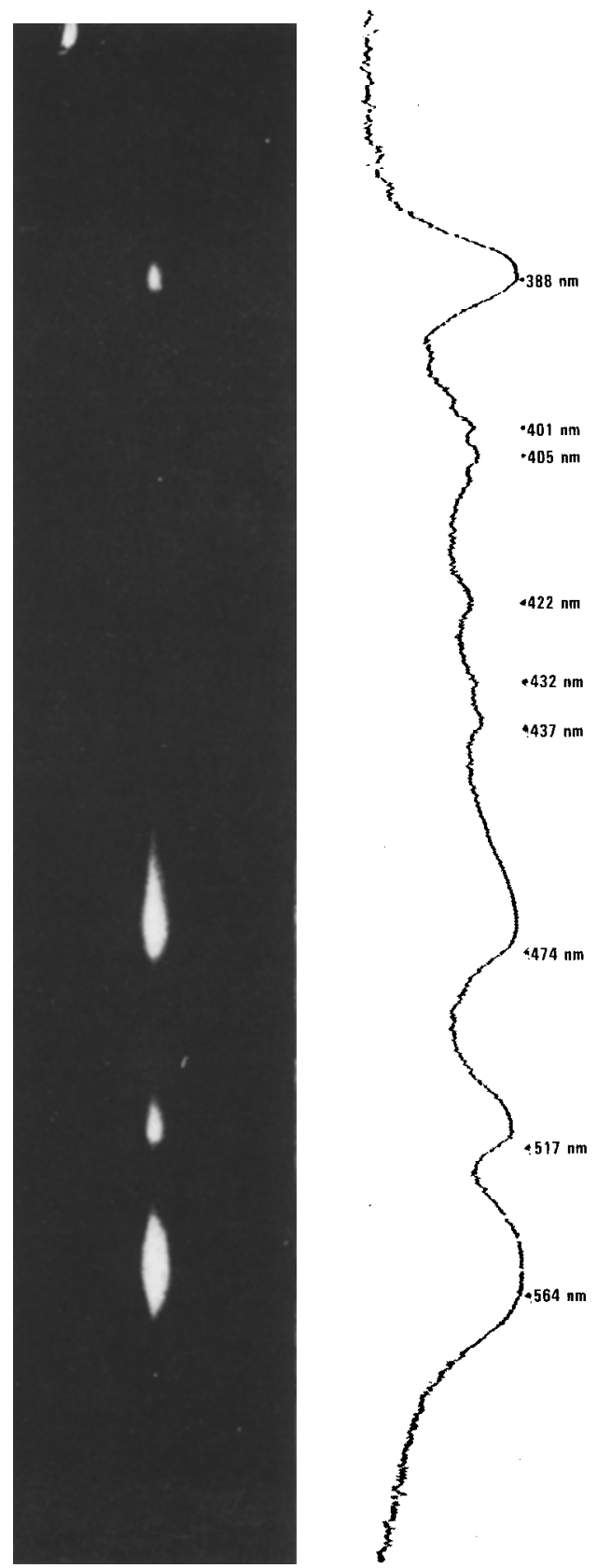

Fig. 9. Spectrum of Halley's comet taken on 1910 May 27 in a two-hour exposure at Yerkes Observatory (Bobrovnikoff, 1927). For identification of emission line species, see Tables VI and XI, and Figure 10.

Halley's 1986 apparition. Indeed this list may well be greatly extended both by remote sensing and by in situ mass spectroscopy during the flybys of the GIOTTO and VEGA spacecraft. 

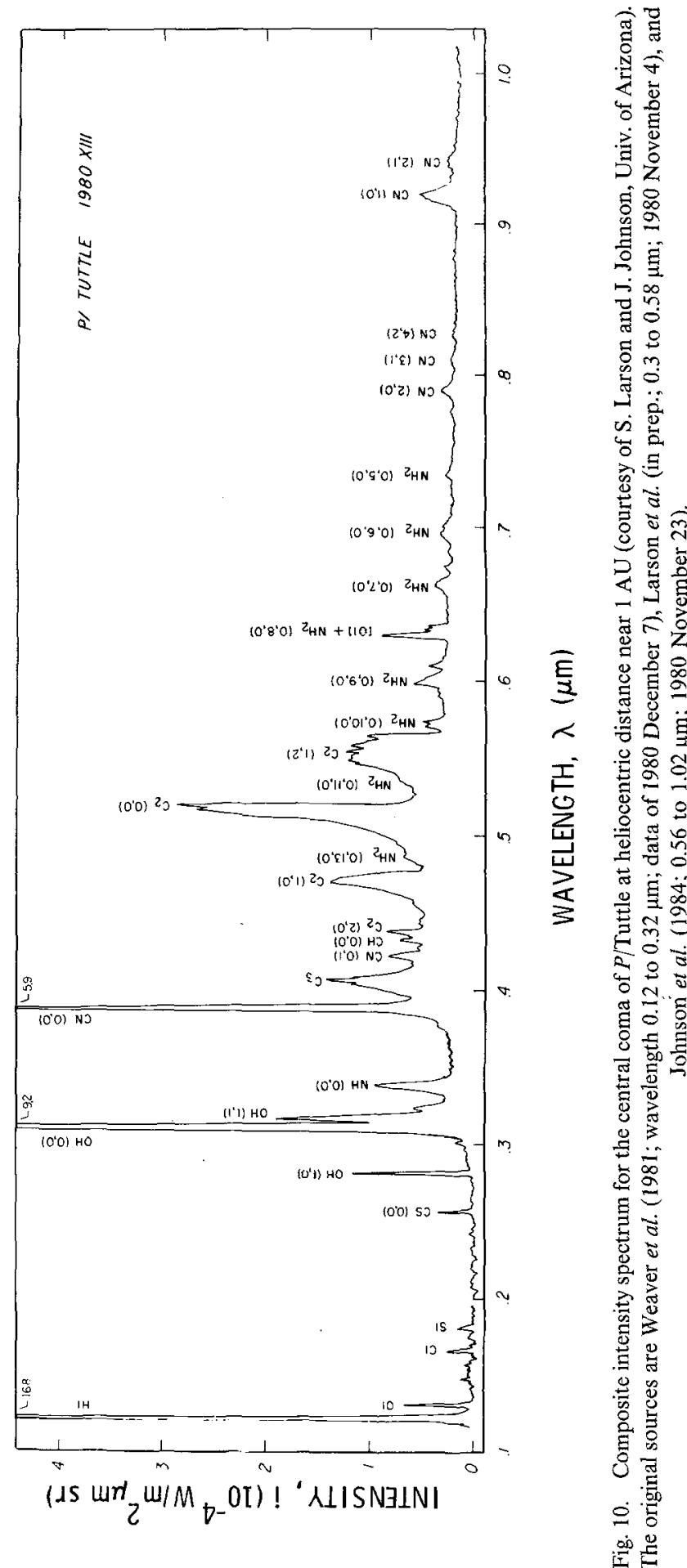
TABLE VII

Chemical species identified in cometary spectra

(tentative identifications in parentheses)

\begin{tabular}{lll}
\hline Atoms & Molecules & Ions \\
\hline $\mathrm{H}$ & $\mathrm{C}_{2}$ & $\mathrm{C}^{+}$ \\
$\mathrm{O}$ & ${ }^{12} \mathrm{C}^{13} \mathrm{C}$ & $\mathrm{Ca}^{+}$ \\
$\mathrm{C}$ & $\mathrm{CH}$ & $\mathrm{CO}^{+}$ \\
$\mathrm{S}$ & $\mathrm{CN}$ & $\mathrm{CH}^{+}$ \\
$\mathrm{Na}$ & $\mathrm{CO}$ & $\mathrm{CN}^{+}$ \\
$\mathrm{K}$ & $\mathrm{CS}$ & $\mathrm{N}_{2}^{+}$ \\
$\mathrm{Ca}$ & $\mathrm{NH}$ & $\mathrm{CO}_{2}^{+}$ \\
$\mathrm{V}$ & $\mathrm{OH}$ & $\mathrm{H}_{2} \mathrm{O}^{+}$ \\
$\mathrm{Mn}$ & $\mathrm{C}_{3}$ & $\mathrm{H}_{2} \mathrm{~S}^{+}$ \\
$\mathrm{Fe}$ & $\mathrm{NH}_{2}$ & $\mathrm{OH}^{+}$ \\
$\mathrm{Co}$ & $\mathrm{H}_{2} \mathrm{O}$ & \\
$\mathrm{Ni}$ & $\mathrm{HCN}$ & \\
$\mathrm{Cu}$ & $\mathrm{CH}_{3} \mathrm{CN}$ & \\
& $\mathrm{S}_{2}$ & \\
& $\mathrm{HCO}$ & \\
& $\mathrm{NH}_{3}$ & \\
& $\left(\mathrm{H}_{2} \mathrm{CO}\right)$ & \\
& $\left(\mathrm{NH}_{4}\right)$ & \\
\hline
\end{tabular}

\subsection{Chemical Reactions}

A strong motivation for the study of cometary atmospheres arises from the fact that it provides the only presently available indicator for the chemical composition of cometary nuclei. However, as is seen from Table VII, most of the observed species (with a few exceptions, e.g., $\mathrm{H}_{2} \mathrm{O}, \mathrm{HCN}, \mathrm{CH}_{3} \mathrm{CN}$, and $\mathrm{NH}_{3}$ ) are chemically unstable radicals which are clearly not stored in the nucleus as such, but which are (together with the ions) presumably the UV photo-destruction fragments of more stable 'parent molecules' that initially sublimate from the nucleus (as first proposed by Wurm, 1943). The situation is further complicated by the continuous reshuffling of the chemical species by rapid gas phase reactions in the collision-dominated inner coma. Ion-molecule reactions involving mass transfer, which are mostly exothermic, play a crucial role in this chemical processing of the inner coma (Oppenheimer, 1974).

All models for the cometary atmosphere and ionosphere start with educated guesses about the chemical composition of the nucleus, and attempt to fit the observations with the resulting model profiles. This is not as hopeless as it seems at first glance because in most, if not all, comets water seems to be the dominant ice in the nucleus, with all other chemical species trapped in cages in the water-ice lattice to form a so-called 'clathrate hydrate', as first proposed by Delsemme and Swings (1952).

Although water has recently been detected via its emission at radio wavelengths, its atmospheric abundance (or its corresponding production rate) has not been directly estimated from that observation. There is, however, a large body of indirect evidence 
pointing to its predominance in the nucleus and inner coma (e.g., Delsemme, 1973), with the strongest support coming from the close correlation of $\mathrm{H}$ and $\mathrm{OH}$ production rates (estimated independently using $\mathrm{L} \alpha$ and $\mathrm{OH}$ emission lines in several recent comets), and from the dominance of these rates over those for other species (see Mendis et al., 1985, for a recent review).

Shimizu (1975) discussed, in some detail, the ion chemistry which would take place following evaporation and photo-ionization from what he calls a 'dirty ice nucleus of the second kind', composed primarily of $\mathrm{H}_{2} \mathrm{O}$ with $\mathrm{N}_{2}$ and $\mathrm{CO}$ as secondary constituents, and of organics (such as $\mathrm{HCN}, \mathrm{CH}_{3} \mathrm{CN}, \mathrm{CH}_{4}$, etc.) as minor constituents. The applicable reaction schemes are illustrated in Figures 11 and 12 . We stress that

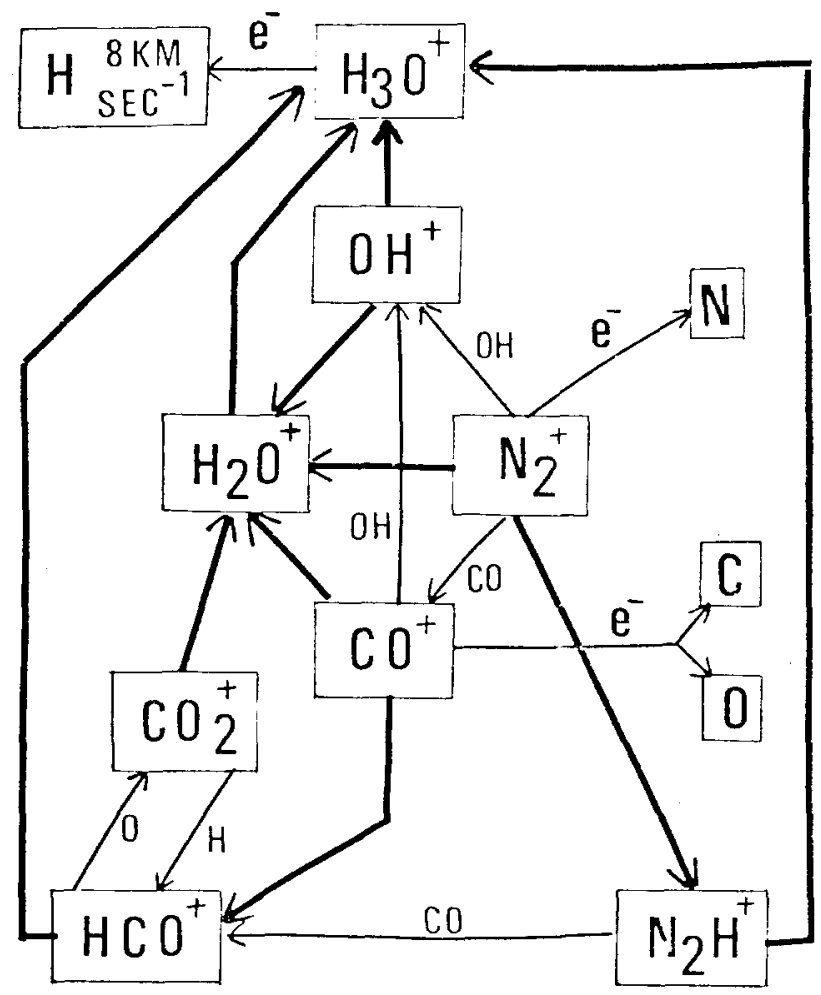

Fig. 11. Ion-molecular reactions in cometary atmospheres for $\mathrm{H}_{2} \mathrm{O}, \mathrm{CO}$, and $\mathrm{N}_{2}$ sequence. Thick arrows are used for reactions with $\mathrm{H}_{2} \mathrm{O}$ and thin arrows for other reactants (symbols are shown beside the arrows). The $\mathrm{H}_{2} \mathrm{O}^{+}, \mathrm{CO}^{+}$, and $\mathrm{N}_{2}{ }^{+}$ions are produced by photoionization of the corresponding neutrals (from Shimizu, 1975; with a minor correction).

since $\mathrm{H}_{2} \mathrm{O}$ is the major atmospheric component and $\mathrm{N}_{2}$ and $\mathrm{CO}$ have high ionization potentials, almost all the ions formed by photo-ionization of the neutral molecules are eventually converted to $\mathrm{H}_{3} \mathrm{O}^{+}$via reactions with $\mathrm{H}_{2} \mathrm{O}$ specified by

$$
\mathrm{H}_{2} \mathrm{O}^{+}+\mathrm{H}_{2} \mathrm{O} \rightarrow \mathrm{H}_{3} \mathrm{O}^{+}+\mathrm{OH},
$$




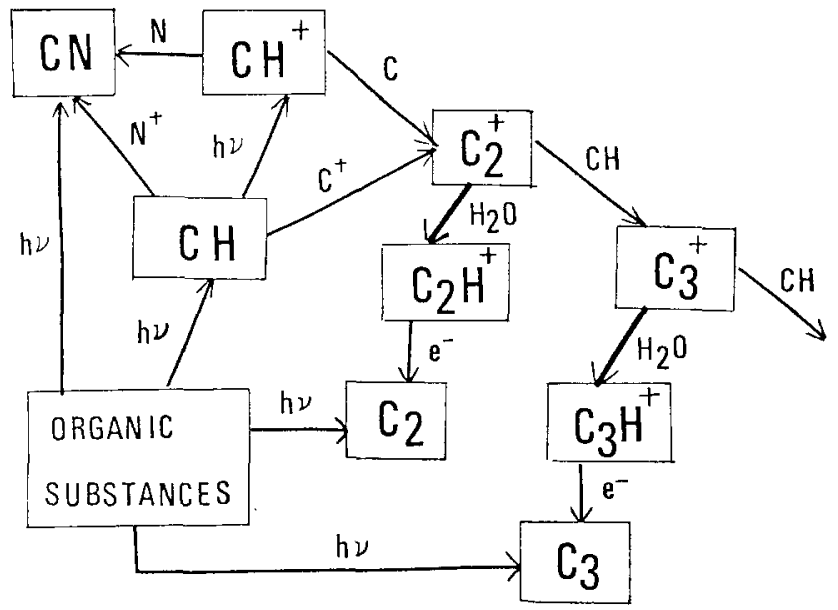

Fig. 12. Ion-molecular reactions in cometary atmospheres for the organic compounds sequence. Symbols for the reactants appear beside the arrows. The organic substances contain $\mathrm{C}_{2} \mathrm{~N}_{2}, \mathrm{C}_{3} \mathrm{H}_{4}, \mathrm{HCN}$, and $\mathrm{CH}_{3} \mathrm{CN}$ (plus small amounts of $\mathrm{CH}_{4}, \mathrm{NH}_{3}$, etc.); $h v$ means either photodissociation or photoionization (from Shimizu, 1975).

$$
\left.\begin{array}{l}
\mathrm{OH}^{+}+\mathrm{H}_{2} \mathrm{O} \rightarrow \mathrm{H}_{3} \mathrm{O}^{+}+\mathrm{O}, \\
\mathrm{CO}^{+}+\mathrm{H}_{2} \mathrm{O} \rightarrow \mathrm{HCO}^{+}+\mathrm{OH} \\
\mathrm{HCO}^{+}+\mathrm{H}_{2} \mathrm{O} \rightarrow \mathrm{H}_{3} \mathrm{O}^{+}+\mathrm{CO}
\end{array}\right\},
$$

and

$$
\left.\begin{array}{l}
\mathrm{N}_{2}^{+}+\mathrm{H}_{2} \mathrm{O} \rightarrow \mathrm{N}_{2} \mathrm{H}^{+}+\mathrm{OH} \\
\mathrm{N}_{2} \mathrm{H}^{+}+\mathrm{H}_{2} \mathrm{O} \rightarrow \mathrm{H}_{3} \mathrm{O}^{+}+\mathrm{N}_{2}
\end{array}\right\}
$$

Consequently, $\mathrm{H}_{3} \mathrm{O}^{+}$is expected to dominate the innermost parts of the ionosphere, before it is removed by the fast dissociative recombination reactions

$$
\left.\begin{array}{l}
\mathrm{H}_{3} \mathrm{O}^{+}+e^{-} \rightarrow \mathrm{H}_{2} \mathrm{O}+\mathrm{H} \\
\mathrm{H}_{3} \mathrm{O}^{+}+e^{-} \rightarrow \mathrm{OH}+\mathrm{H}+\mathrm{H}
\end{array}\right\}
$$

Aiken (1974) and Shimizu (1975) also noted that in the presence of significant quantities of $\mathrm{NH}_{3}$ the ion composition of the inner coma would be shifted towards the ammonium ion via the proton transfer reaction

$$
\mathrm{H}_{3} \mathrm{O}^{+}+\mathrm{NH}_{3} \rightarrow \mathrm{NH}_{4}^{+}+\mathrm{H}_{2} \mathrm{O} \text {. }
$$

In fact, Ip (1981) has shown that if the nuclear abundance ratio $\left[\mathrm{NH}_{3}\right] /\left[\mathrm{H}_{2} \mathrm{O}\right]$ exceeds about 0.1 , then $\mathrm{NH}_{4}{ }^{+}$should dominate the inner coma within about $1000 \mathrm{~km}$ of the nucleus.

Detailed chemical models of the cometary atmosphere using much more extensive chemical reaction networks have subsequently been calculated by several authors (e.g., 
Giguere and Huebner, 1978; Huebner and Giguere, 1980; Mitchell et al., 1981; and Biermann et al., 1982), with the most comprehensive reaction network considered so far being that of Mitchell et al. (1981), who include 1192 reactions among 128 species in one system.

As an example, Figures 13 and 14 present results using 'composition 5' of Huebner and Giguere (1980). Despite the unacceptably large ratio $[\mathrm{CO}] /\left[\mathrm{H}_{2} \mathrm{O}\right]=0.6$ used by

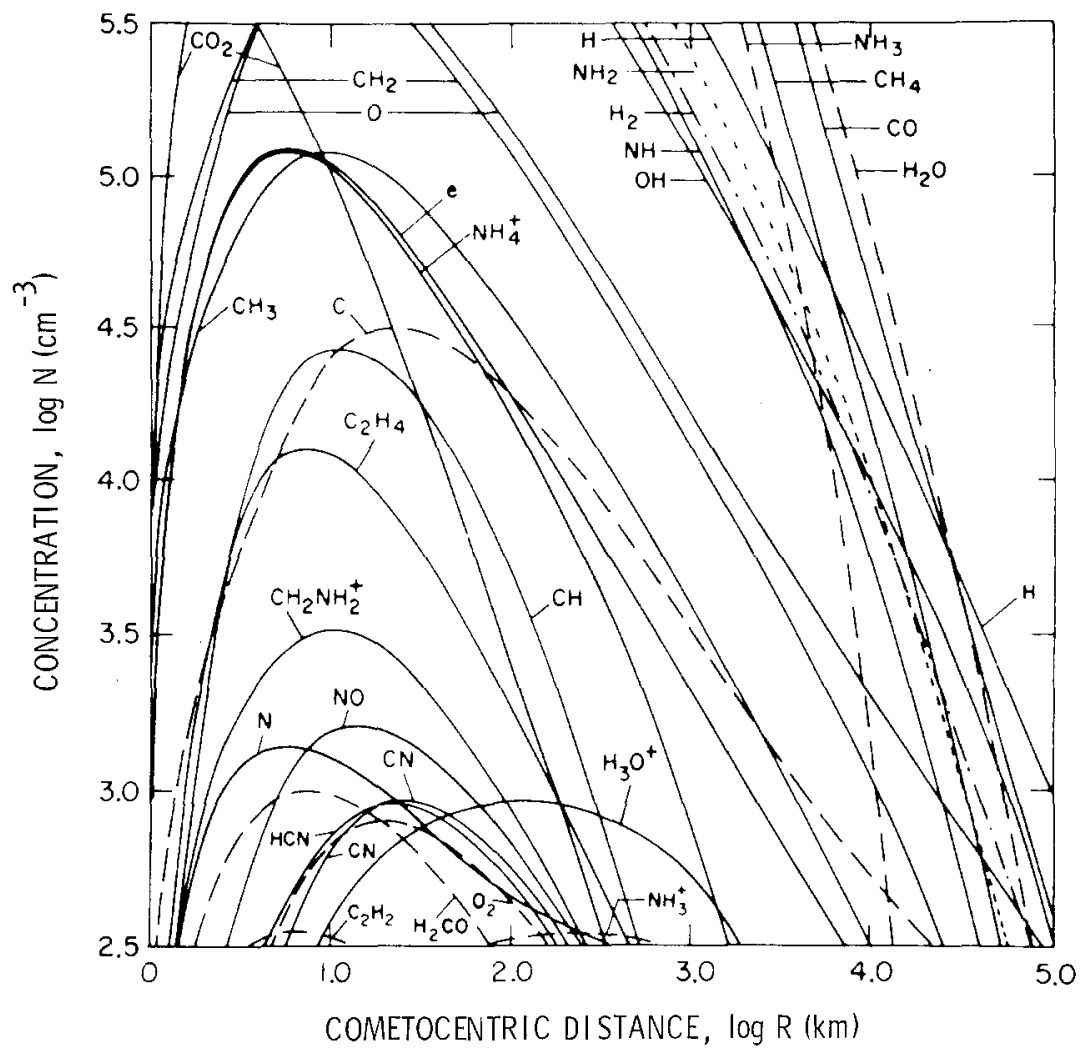

Fig. 13. Radial profiles using ratios $0.6,0.23$, and 0.23 for $\mathrm{CO}, \mathrm{CH}_{4}$ and $\mathrm{NH}_{3}$ to $\mathrm{H}_{2} \mathrm{O}$ by number at the surface, for heliocentric distance $r=1 \mathrm{AU}$ (from Huebner and Giguere, 1980).

these authors (see Mendis et al., 1985, for a detailed criticism), this model, in common with all the previously mentioned chemical models, falls short by over an order of magnitude in predicting the observed $\left[\mathrm{CO}^{+}\right] /\left[\mathrm{H}_{2} \mathrm{O}^{+}\right]$ratio in Comet Kohoutek (1973 XII). It now appears that a plausible explanation for this observed ratio is to invoke the chemical differentiation that is expected in the nucleus' evolving mantle (cf. Section 5.1; Houpis et al., 1985; and Mendis, 1985).

Several authors have also studied the combined dynamics and thermodynamics of the cometary atmosphere, while minimizing the chemistry. A comprehensive review of hydrodynamic models of the collision-dominated inner coma is given by Mendis et al. (1985). 


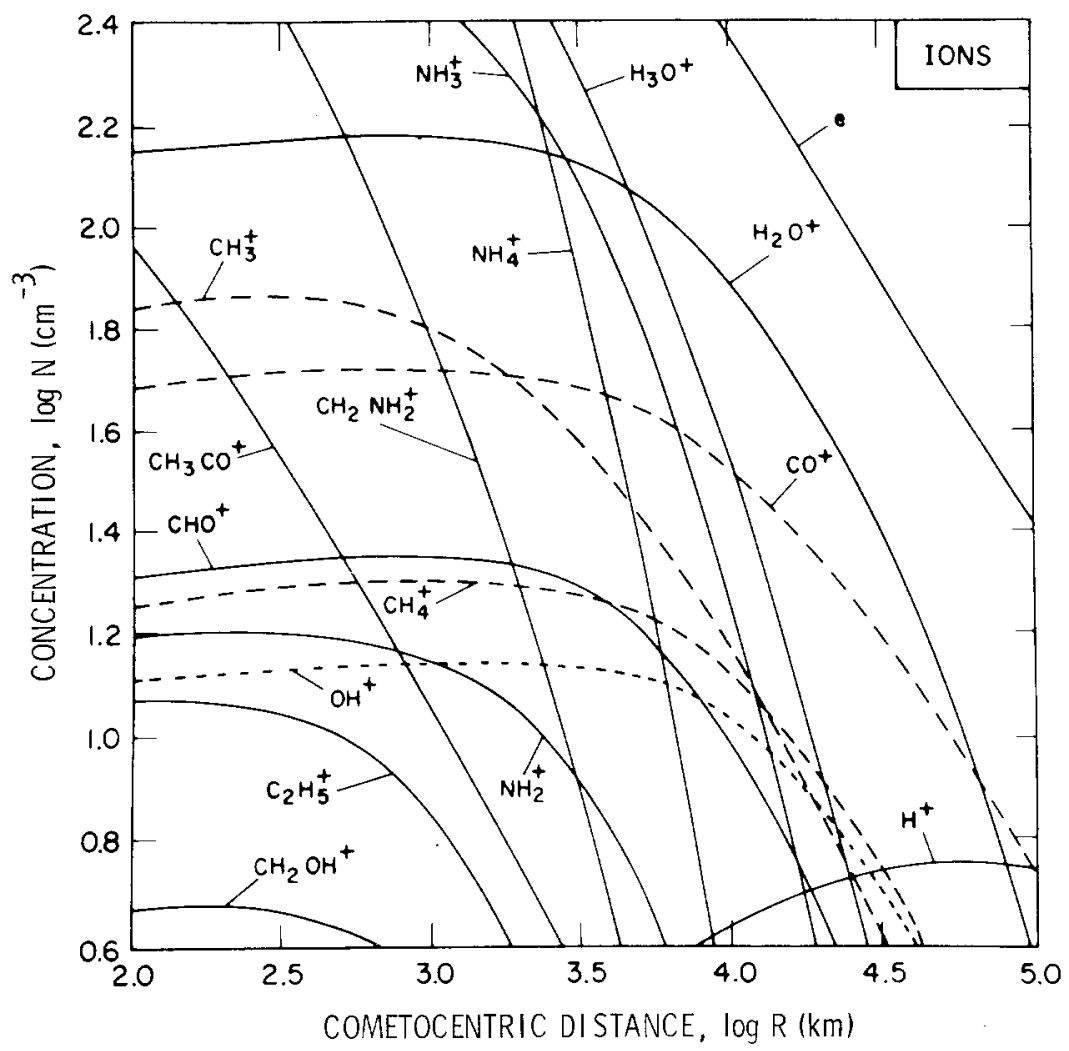

Fig. 14. Ion radial profiles for the model of Figure 13 (from Huebner and Giguere, 1980).

\subsection{Distributions of Gas MOLECULES}

For each of the above reactions a first-order treatment of the distributions of the 'parent' and 'daughter' molecules can be made by combining radial outflow with simple scale lengths for destruction and/or creation. Such scale lengths are most useful beyond the collision zone, for a region within which photolytic processes dominate. To account for situations in which concentrations, reaction cross sections, rates and lifetimes may be poorly known, or in which competing processes make the analysis complex, values for scale lengths which might be obtained from detailed computations (such as those in Figures 13 and 14) can often be better determined from measurements of changes in emission line strengths with position in the coma. On this basis, as originally developed by Haser (1957), the molecular concentrations $\left(\mathrm{m}^{-3}\right)$ can be expressed in the form

$$
n_{p}=\frac{Q_{p}}{4 \pi R^{2} u_{p}} e^{-R / l_{p}}
$$

and

$$
n_{d}=\frac{Q_{p}}{4 \pi R^{2} u_{d}} \frac{l_{d}}{l_{d}-l_{p}}\left(e^{-R / l_{d}}-e^{-R / l_{p}}\right)
$$


Here the symbols $Q, u$, and $l$ represent production rate $\left(\mathrm{s}^{-1}\right)$, radial outflow velocity $\left(\mathrm{m} \mathrm{s}^{-1}\right)$, and scale length $(\mathrm{m})$, respectively, and subscripts $p$ and $d$ distinguish parent from daughter molecules. This approach has been especially useful in interpreting the distributions of gas molecules having emission spectra in the visible region; several of these are discussed individually by A'Hearn (1982; cf. also Section 9.1), and they form the basis for the determination of production rates $Q_{p}$ for some parent molecules. In most cases the daughter scale lengths exceed those for the parent, as illustrated by the sample values in Table VIII for several species observed at visible wavelengths. Within the collision zone (having a radius of a few thousand $\mathrm{km}$ ), the Haser theory is a poor approximation for the molecular distributions, since there most radicals are formed and destroyed by several processes besides simple photodissociation (Huebner et al., 1982). Often the scale lengths used to approximate the average results of competing processes have no simple physical interpretation, and they are really useful at all only if the point of evaluation is not inside the region where the processes occur. For a more detailed approach, see Festou (1981a, b).

The distribution of atomic hydrogen in a cometary coma is unique among those for a gaseous species, both because it is the most abundant element in the nucleus (by number), and because the kinetic energy available from photodissociation yields outflow velocities for these very light atoms much larger than the thermal speeds available in the collision-dominated inner coma. The interpretation of UV spectra has been central to the establishment of both these conclusions (Keller, 1976). The hydrogen atoms come from the direct dissociation of water $\left(\mathrm{H}_{2} \mathrm{O}+h v \rightarrow \mathrm{H}+\mathrm{OH}\right)$ and from the secondary dissociation of hydroxyl $(\mathrm{OH}+h v \rightarrow \mathrm{O}+\mathrm{H})$. In each of these reactions the responsible incident UV photons (mostly solar $\mathrm{L} \alpha$ ) provide excess kinetic energy primarily to the lighter $\mathrm{H}$ atoms. Most of the water is predissociated, leading to $\mathrm{H}$ atoms with velocities distributed around a mean of $20 \mathrm{~km} \mathrm{~s}^{-1}$. More energetic photons yield dissociation in excited states and $\mathrm{H}$ atoms with even larger velocities. For the $\mathrm{OH}$, predissociation yields $\mathrm{H}$ atoms with velocities near $8 \mathrm{~km} \mathrm{~s}^{-1}$ and dissociation faster components. Thus there are, roughly speaking, two components of hydrogen atoms, the high velocity component with $u$ near $20 \mathrm{~km} \mathrm{~s}^{-1}$ (mainly from water) and the low velocity component with $u$ near $8 \mathrm{~km} \mathrm{~s}^{-1}$ (from $\mathrm{OH}$ ). These high velocities and the relatively long lifetime near $2 \times 10^{6} \mathrm{~s}$ at $r=1 \mathrm{AU}$ make the hydrogen coma at least one order of magnitude larger than the UV coma of the heavier elements $O$ and $C$. Such elements can be traced beyond $1 \mathrm{Gm}$ (already an order of magnitude farther than the visible coma of the radicals). Photometric observations of Bennett $1970 \mathrm{II}$ (Keller and Thomas, 1975) revealed a hydrogen coma extending more than $50 \mathrm{Gm}(0.33 \mathrm{AU})$.

Interpretation of these $\mathrm{UV}$ data shows that for $\mathbf{H}$ the radial outflow model with parent and daughter scale lengths is inadequate, and that a model which combines the radial outflow velocity vectorially with the isotropic velocity distribution from the photodissociation is required (Festou et al., 1979; Festou, 1981a,b). Additionally the hydrogen atoms are subject to radiation pressure (again primarily from $L \alpha$ ) which (just as for the dust, as in Section 7.4) imparts both asymmetry and curvature to the isophotes of the large $\mathbf{H}$ atom coma (Keller, 1976; cf. also Section 9.2). 
TABLE VIII

Sample scale lengths for seven neutral species observed in cometary comae, evaluated at heliocentric distance $r=1 \mathrm{AU}$. For other values of $r$, the scale lengths are proportional to $r^{2}$ for all entries except $\mathrm{C}_{2}$ $\left(l_{p} \sim r\right)$ and atomic oxygen (the ${ }^{1} D$ metastable excited state from photodissociation of $\mathrm{H}_{2} \mathrm{O}$ decays via a forbidden transition at $630 \mathrm{~nm}$ to the ground state, so that $l_{d}$ is independent of $r$; cf. Table XI). See A'Hearn (1982) for individual discussions for each molecule.

\begin{tabular}{llll}
\hline $\begin{array}{l}\text { Daughter } \\
\text { species }\end{array}$ & $\begin{array}{l}\text { Parent } \\
\text { scale length } \\
l_{p}(\mathrm{~km})\end{array}$ & $\begin{array}{l}\text { Daughter } \\
\text { scale length } \\
l_{d}(\mathrm{~km})\end{array}$ & Reference \\
\hline $\mathrm{OH}$ & $8 \times 10^{4}$ & $2 \times 10^{5}$ & Festou (1981b) \\
$\mathrm{NH}$ & $5.8 \times 10^{3}$ & $4.3 \times 10^{5}$ & Combi (1978) \\
$\mathrm{CN}$ & $1.2 \times 10^{4}$ & $3.0 \times 10^{5}$ & A'Hearn and Cowan (1980) \\
$\mathrm{C}_{3}$ & $2.5 \times 10^{3}$ & $6.0 \times 10^{4}$ & A'Hearn and Cowan (1980) \\
$\mathrm{C}_{2}$ & $3.8 \times 10^{4}$ & $1.2 \times 10^{5}$ & A'Hearn and Cowan (1980) \\
$\left.\mathrm{O}^{1}{ }^{1} D\right)$ & $8 \times 10^{4}$ & $1.5 \times 10^{2}$ & Festou (1981b); Spinrad (1982) \\
$\mathrm{NH}_{2}$ & $7 \times 10^{3}$ & $1.0 \times 10^{4}$ & Johnson et al. (1984) \\
\hline
\end{tabular}

The complexity of the reactions discussed in Section 6.2 (in the inner coma), and of the photodissociation, photoexcitation and photoemission processes which apply to various gas species (particularly $\mathrm{OH}$ and $\mathrm{O}$; cf. Table VIII for an example) has enabled useful insights into the physics and chemistry of the coma to be derived from UV, visible, IR, and radio spectra and spatial distributions. Recent discussions of the relevant details are provided by Feldman (1982), A'Hearn (1982), Jackson (1982), Huebner et al. (1982), Festou (1984), and Crovisier (1985).

\subsection{A GAS MODEL FOR HALLEY}

Here we will confine ourselves to the most recent and comprehensive of the chemical and hydrodynamic models, those that have been developed by Marconi and Mendis $(1983,1984)$ specifically for Halley's comet at $r=0.89 \mathrm{AU}$ (the heliocentric distance at the GIOTTO encounter). These authors consider the outgassed atmosphere of a dirty-clathrate cometary nucleus composed of $85 \% \mathrm{H}_{2} \mathrm{O}, 10 \% \mathrm{CO}_{2}$, and $5 \% \mathrm{~N}_{2}$ by number, with a non-volatile ('dust') to volatile ('ice') ratio 0.5 by mass. The authors point out that from a dynamic and thermodynamic point of view, the neglect of other trace parent molecules is justified because both the heating and cooling of the atmosphere are dominated by the abundant $\mathrm{H}_{2} \mathrm{O}$, via its photolysis and its rotational transitions. The only other important species from this point of view is the dust that is entrained by the radially outflowing gas. The partial choking of the gas outflow by the dust drag causes the gas to start off subsonically at the nucleus' surface and to smoothly traverse the sonic point close to the surface (as first demonstrated by Probstein, 1969, and discussed in Section 5). Also, the circumnuclear dust plays a central role in the radiative transfer of the continuum solar radiation in the inner coma. On the one hand, there is the direct absorption of the incident solar radiation by this dust. But this is more than compensated for by the diffuse radiation fields due to multiple scattering and thermal re-radiation by this extensive halo of dust that is considerably hotter than the icy nucleus. 
Both the gas and dust production rates from the nucleus are increased over what they would have been had the dust been absent, due to the net enhancement of the insolation, a point already made by Hellmich (1981) and by Weissman and Kieffer (1981).

Marconi and Mendis (1983, 1984) obtained a steady-state, transonic, multi-fluid solution of the dynamical and thermal structure of this dusty gas atmosphere by solving the simultaneous set of differential equations representing conservation of number density, momentum, and energy (cf. Equation (5.7) through (5.12)), with the transfer of the UV solar radiation responsible for the major photolytic processes, and the continuum radiation responsible for the direct and indirect heating of the nucleus. While the heavy neutral and ion species are treated as a single fluid in the collision-dominated inner coma, the photo-produced atomic hydrogen (which acquires the bulk of the kinetic energy released during $U V$ photolysis of the dominant $\mathrm{H}_{2} \mathrm{O}$ species) is treated separately. The $\mathrm{H}$ atoms exhibit a thermalized component (having its own temperature and velocity) and a pre-thermal component (regarded as a fast, cold, free-streaming beam) which gradually thermalizes by elastic collisions with heavier species. Also, the photoelectrons (which thermalize rapidly among themselves, but which exchange energy inefficiently with the ions and neutrals) are considered as a separate fluid.

The diffuse radiation fields due to dust, which were neglected in the earlier model (Marconi and Mendis, 1983) were included using a 3-stream approximation based on the shadowing geometry of the nucleus in the later model (Marconi and Mendis, 1984). They considered the nucleus as a sphere of radius $R_{N}=3 \mathrm{~km}$, with bolometric Bond albedo $A=0.225$ and infrared emissivity $\varepsilon_{N}=(1-A)=0.775$. They also assumed low bulk density dust grains $\left(\rho=1 \mathrm{~g} \mathrm{~cm}^{-3}\right)$ with the optical properties of magnetite, and a single grain size (radius $a=0.5 \mu \mathrm{m}$ ). Even though the development of a dust model would require major improvements in these assumptions (as in Sections 7 and 9), results for velocities, temperatures, etc., for the gas are only modestly affected.

Some results of this latter calculation (Marconi and Mendis, 1984) are shown in Figures 15 through 19 . Figures 15 and 16 show the velocity and temperature profiles of the gas and dust in the thin subsonic boundary layer, which extends only to about $140 \mathrm{~m}$ from the nuclear surface. The corresponding profiles in the supersonic region of the collision-dominated atmosphere are shown in Figures 17 and 18.

Of particular interest are the temperature profiles. While the temperature of the $0.5-\mu \mathrm{m}$ radius dust grains, which starts off at about $380 \mathrm{~K}$, remains relatively constant (at $413 \mathrm{~K}$ ) throughout the outer atmosphere, the temperatures for all the gaseous species go through a broad minimum, due to the combined effects of expansion cooling and the dominance of infrared cooling over photolytic heating in the inner coma. It was shown that minima would exist in the temperature profiles even if the IR cooling did not take place, although the minima in that case would be around 20 to $30 \mathrm{~K}$, rather than the 3 to $4 \mathrm{~K}$ when the IR cooling is included. It is also clear that the thermal coupling between the electrons and the heavy gaseous species (including the ions) breaks down beyond about $100 \mathrm{~km}$, first gradually and then sharply around $2000 \mathrm{~km}$. Such thermal decoupling between electrons and other species is a common phenomenon in gravitationally bound planetary atmospheres too. While the electron temperature rises to about 


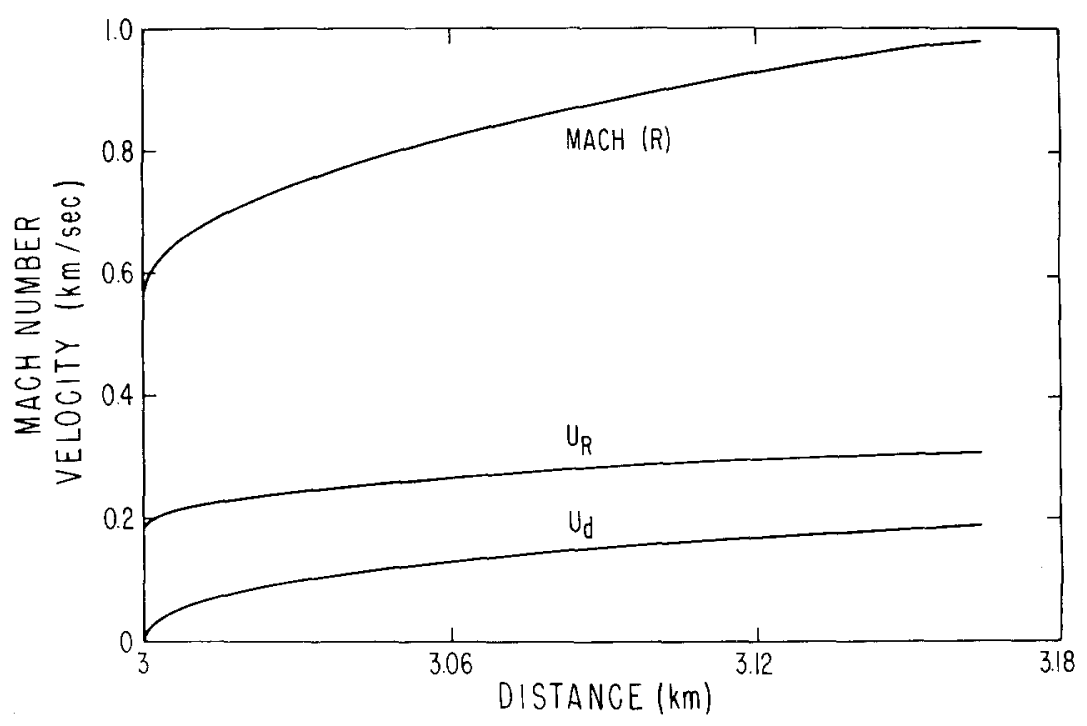

Fig. 15. Radial profiles of the velocity of heavy neutral and ion species $(R)$ and the dust $(d)$, as well as Mach number for the heavy species in the subsonic region of the Halley model in Section 6.4 (from Marconi and Mendis, 1984).

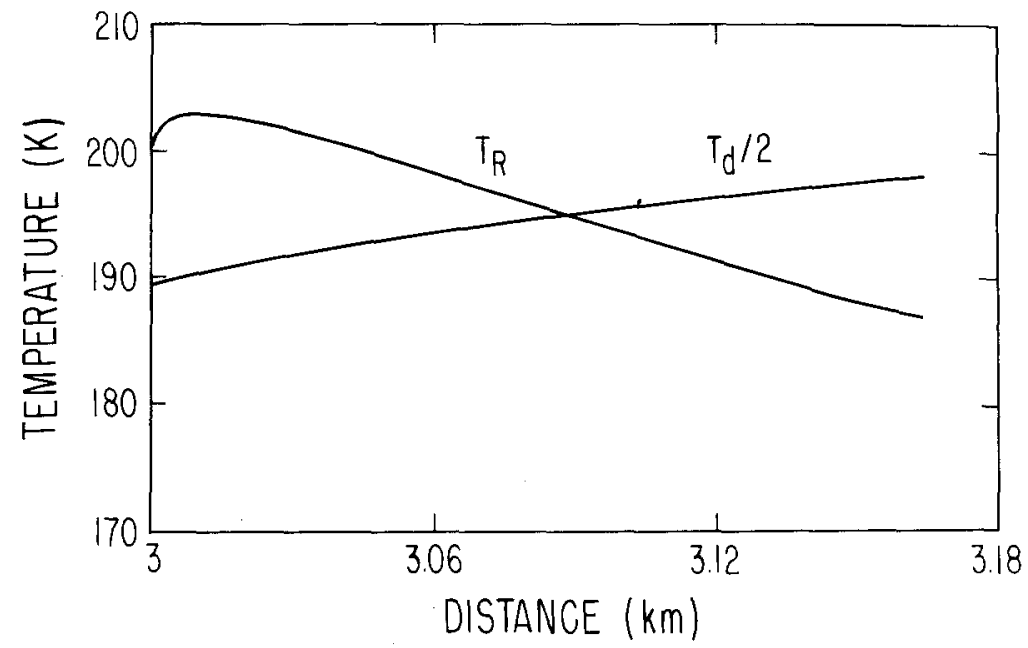

Fig. 16. Radial profiles of the temperature of heavy species $(R)$ and dust $(d)$ in the subsonic region of the Halley model from Section 6.4 (from Marconi and Mendis, 1984).

$32000 \mathrm{~K}$ at $R=10^{4} \mathrm{~km}$ in the absence of electron thermal conductivity (see also Ashihara, 1978), it rises to a maximum of only $7000 \mathrm{~K}$ when the electron thermal conductivity is included. These high electron temperatures have a significant effect on electron densities in the outer coma. Since the dissociative recombination of electrons and ions is less efficient at higher temperatures, the electron density is enhanced over what it would have been if the atmosphere, including the electrons, were isothermal (as is generally assumed in chemical models). 


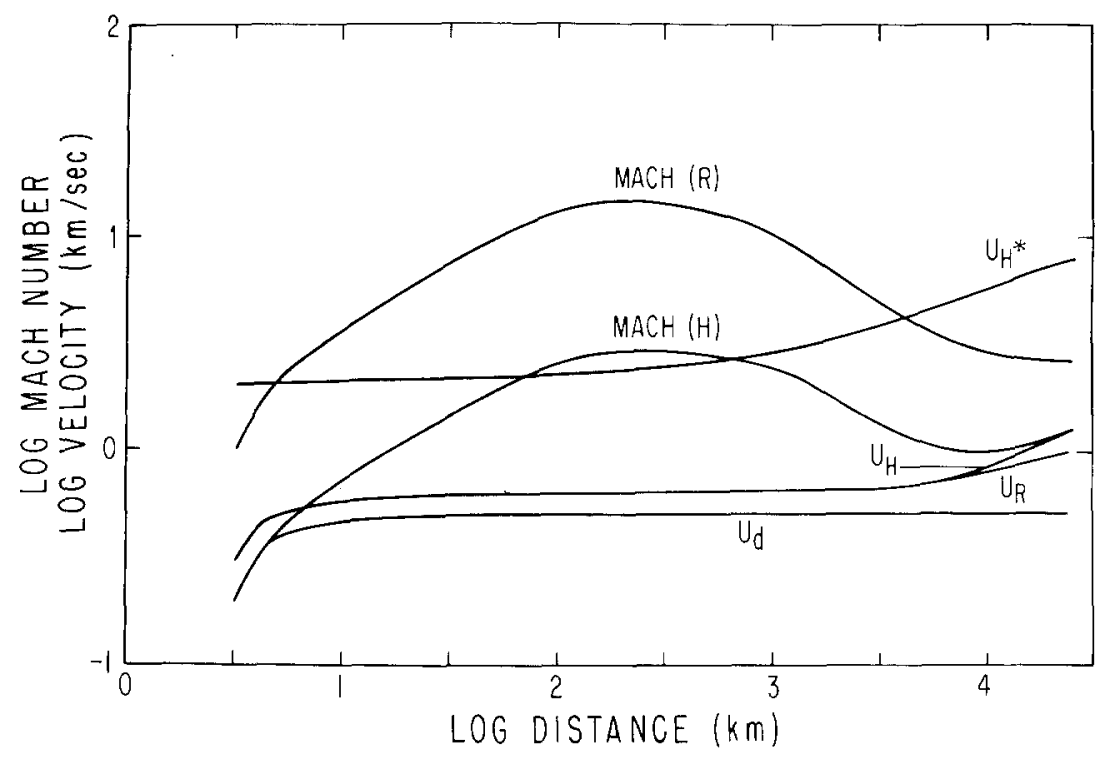

Fig. 17. Radial profiles of the Mach numbers of the heavy species (R) and the thermal hydrogen $(H)$ and the velocities of $\mathrm{R}, \mathrm{H}$, the prethermal hydrogen $\left(\mathrm{H}^{*}\right)$, and the dust (d), in the supersonic region of the Halley model from Section 6.4 (from Marconi and Mendis, 1984).

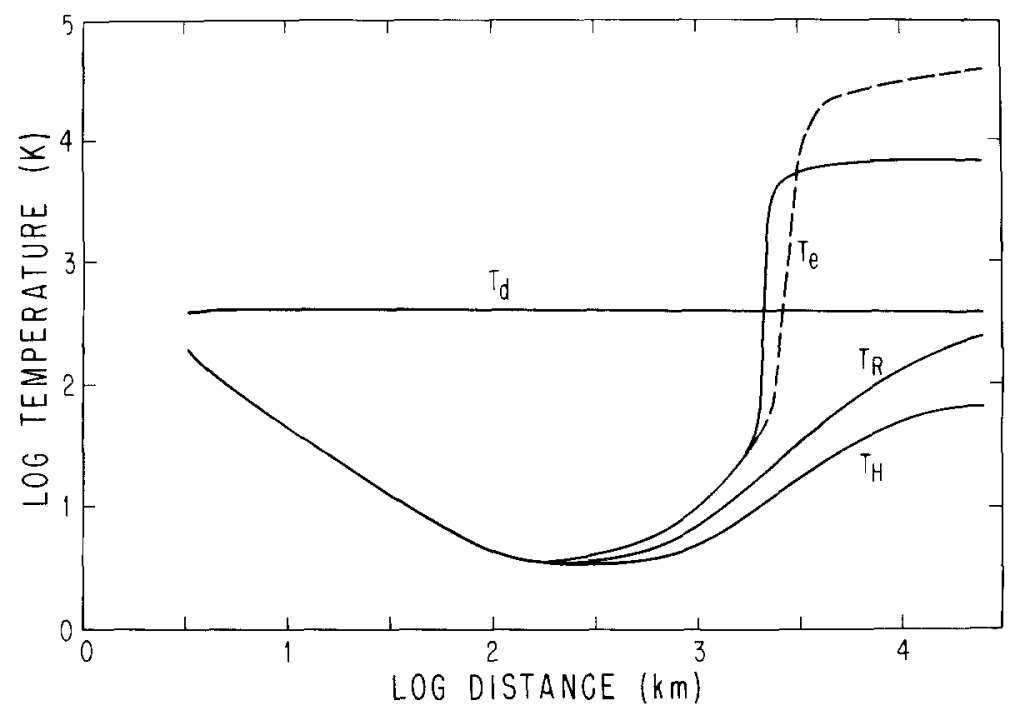

Fig. 18. Radial profiles of the temperatures of the dust (d), the heavy species ( $R$ ), the thermalized hydrogen $(\mathrm{H})$, and the electrons (e) in the supersonic region of the Halley model of Section 6.4 (from Marconi and Mendis, 1984).

The radiative transfer between the relatively hot circumnuclear dust (which radiates essentially in the range $1-10 \mu \mathrm{m}$ ) and the dominant $\mathrm{H}_{2} \mathrm{O}$-molecule (which has strong rotational transitions in this wavelength range) has been neglected in these calculations. Recent preliminary results by Marconi and Mendis (1985) indicate that this could cause 
significant heating in the innermost coma and thereby elevate the gas temperatures to values above those shown in Figure 18. In these latest calculations the ionic species and the neutral species are considered as different fluids, which also leads to an increase in the ion temperature in the inner coma. A similar result was obtained earlier by Korosmezey (1984) using a simplified dust-free model. However, these results must be considered tentative because all the possible modes of ionic cooling in the inner coma have not been adequately considered yet. Yamamoto and Ashihara (1984) have discussed the possible recondensation of the $\mathrm{H}_{2} \mathrm{O}$ vapor to form minute ice grains in the vicinity of the nucleus. If this indeed does happen it would provide an additional heat source in the innermost coma. However, at present this recondensation process remains an open question because the IR heating by the dust in the innermost coma, discussed earlier, was not included in these calculations.

Finally, Figure 19 shows radial profiles of the mean intensities of direct and diffuse radiation from the Sun and dust (cf. Section 9.3). The enhanced insolation of the

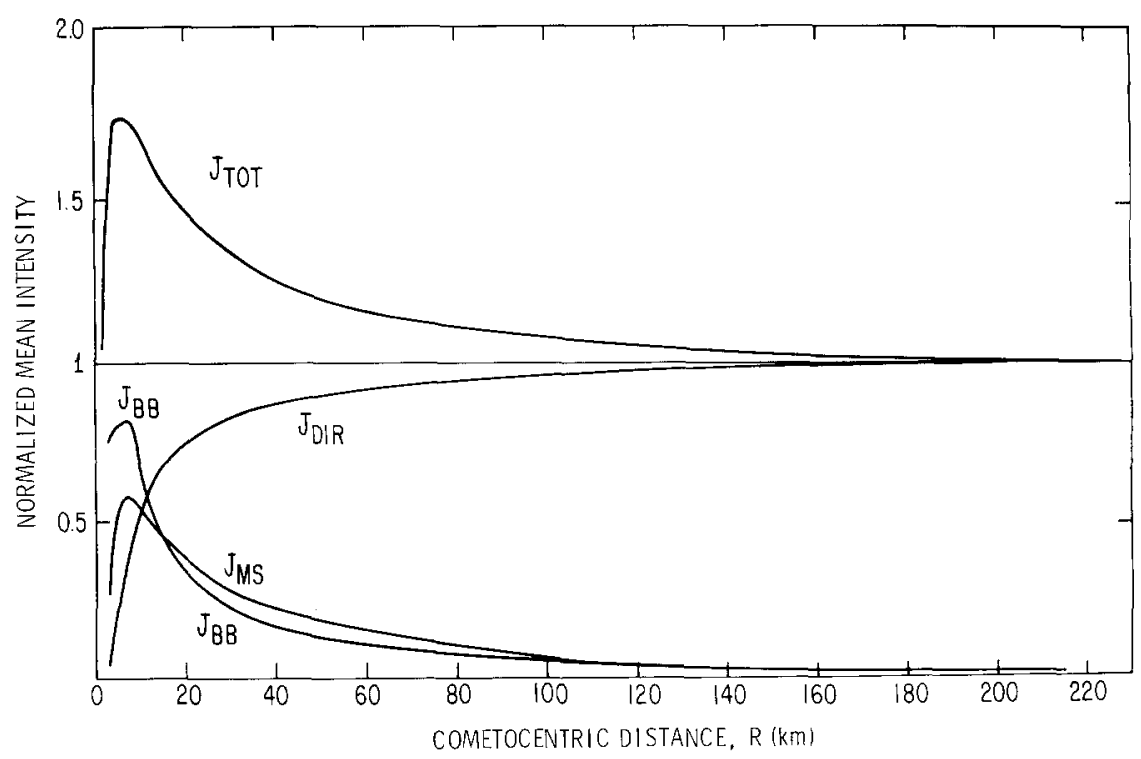

Fig. 19. Radial profiles of the normalized mean intensity of radiation in the inner coma of the Halley model of Section 6.4 (from Marconi and Mendis, 1984). The subscripts DIR, MS, and BB identify direct solar radiation, multiply scattered radiation, and black-body thermal re-radiation by the circumnuclear dust.

nucleus caused by the diffuse radiation fields elevates the total production rate of water at this heliocentric distance $(r=0.89 \mathrm{AU})$ to $5.0 \times 10^{29} \mathrm{~s}^{-1}$. This value is comparable to the value calculated using the semi-empirical model for gas and dust production from Halley's postperihelion magnitude (Section 3.2 and Table V). 


\section{Cometary Dust}

Until space probes provide us with direct measurements of the composition, density and mass distribution of cometary dust grains, our information on the nature of the grains must come from optical and infrared remote sensing data and from studies of micrometeorites collected in the Earth's stratosphere. The following sections describe the optical, chemical and physical properties of such dust grains, and extend the model of their dynamics (cf. Section 5.2) to the outer coma and beyond.

\subsection{OPTICAL AND INFRARED PROPERTIES}

Remote sensing measurements of electromagnetic radiation from cometary dust grains show that the grains from different comets display generally similar properties, such that any dependences of albedo, emissivity and polarization on wavelength, grain size, grain temperature, scattering angle, and heliocentric distance (at least for $r<2 \mathrm{AU}$ ) do not appear to vary from comet to comet. Such measurements provide data for the entire ensemble of dust grains within an observing aperture, whereas individual grains may of course have optical properties which differ from grain to grain.

Wide bandpass data at visual wavelengths (e.g., $U, B$, and $V$ magnitudes) are not useful for dust observations in active comets because they are contaminated by gaseous emission. Rather, the sunlight scattered from the dust must be observed at continuum wavelengths using spectrophotometry or carefully chosen narrow-band filter photometry. Such measurements reveal that the color of the scattered light is neutral to somewhat red at wavelengths 0.36 to $2.2 \mu \mathrm{m}$. In the near-infrared strong gaseous emission bands are absent, so that measurments in the $J, H$, and $K$ bands (wavelengths near 1.25, 1.65, and $2.25 \mu \mathrm{m}$ ) are useful. Typical colors are $J-H=0.44 \pm 0.10$ and $H-K=0.15 \pm 0.08$. The lack of observable Rayleigh scattering implies that the contribution from grains much smaller than these near infrared wavelengths is minor, i.e., that the typical grain diameter $2 a$ exceeds about $1 \mu \mathrm{m}$. For infrared wavelengths larger than about $3.5 \mu \mathrm{m}$, thermal emission from the dust dominates cometary radiation. Here the spectra commonly indicate temperatures considerably higher than for a theoretical blackbody in equilibrium, suggesting absorbing dust grain size smaller than the wavelength (cf. Section 7.2).

Useful, quantitative interpretation of observational data for radiation scattered and emitted by cometary dust requires careful definition of the relevant variables, among which the efficiencies for scattering, absorption, extinction, and radiation pressure are fundamental. As defined in detail by van de Hulst (1957), Hansen and Travis (1974), and others, these four quantities $\left(Q_{\mathrm{sca}}, Q_{\mathrm{abs}}, Q_{\mathrm{ext}}=Q_{\mathrm{sca}}+Q_{\mathrm{abs}}\right.$, and $\left.Q_{\mathrm{pr}}\right)$ represent the dimensionless ratios of the effective cross sections for the four processes to the geometric cross section of an individual particle, and they may depend on wavelength and on the size, shape, orientation, and material properties (e.g., refractive index) of the particle. Light diffracted by the particle is included in both the scattering and extinction efficiencies, and in the scattering function $\Phi_{s}$, which depends also on scattering angle $\theta_{s}=(\pi-\alpha)$, where the phase angle $\alpha$ is formed by the vertex Sun-dust-observer. 
For individual particles, detailed definitions of albedo are provided by Hanner et al. (1981). The single scattering albedo $A$ is defined as the ratio of the energy scattered in all directions to the total energy removed from a unidirectional incident beam at a given wavelength, in the form

$$
A=\frac{Q_{\text {sca }}}{Q_{\text {ext }}}
$$

In contrast, the geometric albedo $p$ is defined as the ratio of the observed, backscattered flux to that from a white Lambert disc of the same geometric cross section, and this definition is equivalent to

$$
p=\pi A Q_{\mathrm{ext}} \Phi_{\pi} .
$$

Here the scattering function $\Phi_{s}$ is normalized as in Equation (9.13) and the subscript $\pi$ implies evaluation at $\theta_{s}=180^{\circ}=\pi$ radian. Because comets are seldom observed in precisely the anti-solar direction, it is useful to introduce an albedo $A_{p}$ which depends on scattering angle $\theta_{s}$ in the form

$$
A_{p}=\pi A Q_{\mathrm{ext}} \Phi_{s}=p \Phi_{s} / \Phi_{\pi},
$$

such that $A_{p}$ become equal to the geometric albedo $p$ for $\theta_{s}=180^{\circ}$. Average values of the albedo $A_{p}$ for an observed ensemble of cometary dust grains can be derived from simultaneous measurements of scattered and thermal radiation. At near infrared wavelengths (e.g., $J, H$, and $K$ ) and scattering angles 120 to $170^{\circ}$, typical values for $A_{p}$ are 0.02 to 0.05 for short period comets at $r<2 \mathrm{AU}$, comparable to those for the darkest asteroids (Hanner et al., 1985a, b). These small values rule out any large contribution from cold, bright grains such as pure silicates or ices. However, two recent parabolic comets (Bowell, 1982 I, and Cernis, 19831) had $\mathrm{A}_{\mathrm{p}}$ near 0.06, probably indicating the presence of some icy grains (Hanner and Campins, 1985; Hanner, 1984a). If we extrapolate the more typical values to visible wavelengths and backward scattering angles using dependences suggested by a variety of cometary data, we find that $p=0.03 \pm 0.01$ is a reasonable average value for the grains' geometric albedo at $\lambda=0.5 \mu \mathrm{m}$. This low value is consistent with those for dust particles derived from a cometary occultation (Larson and A'Hearn, 1984) and from polarization studies (Myers and Nordsieck, 1984), for particles collected in the stratosphere (Hanner, 1980) and discussed in Section 7.3, and for zodiacal light particles (Lumme and Bowell, 1985).

In priciple the effective dust scattering function $\Phi_{s}$ can be obtained from observations made as the geometry changes. In such observations one must account for the changing quantity of dust in the coma by normalizing the scattered light to simultaneous data for the gas production (assuming a constant dust-to-gas ratio) or for the thermal emission from the dust itself. The latter (preferable) technique, particularly as used by Ney and Merrill (1976), for Comet West 1975n, and by Ney (1982) for Bradfield 1982t, suggests that the scattering function is fairly flat in the range $90^{\circ}<\theta_{s}<150^{\circ}$, and increases by a factor ten as $\theta_{s}$ decreases from 100 to $30^{\circ}$ (observations of the forward scattering peak 


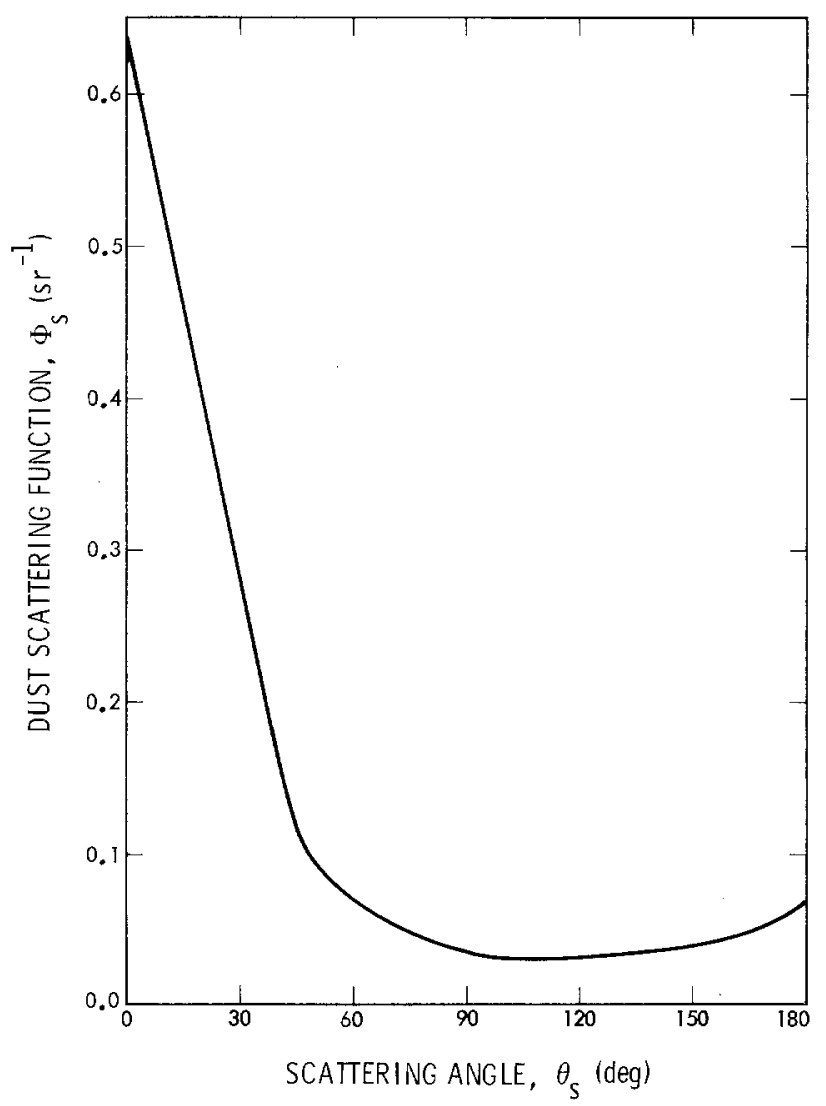

Fig. 20. Candidate scattering function for observed distributions of cometary dust. As derived by Divine (1981b), this function satisfies the normalization of Equation (9.13), and is based on data published by Ney $(1974,1982)$ and others, as extrapolated to scattering angles near 0 and $180^{\circ}$.

for much smaller $\theta_{s}$ are precluded by proximity of the Sun). A modest backscattering peak is also present, such that the value of $\Phi_{s}$ at $\theta_{s}=180^{\circ}$ is about double that at $\theta_{s}=150^{\circ}$ (Millis et al., 1982; Kiselev and Chernova, 1981; Dobrovolsky et al., 1980). The scattering function shown in Figure 20 illustrates these features, which resemble those measured for fluffy, somewhat absorbing grains (Giese, 1980; Hanner et al., 1981). Dependences of $\Phi_{s}$ on wavelength seem to be modest or absent.

As described by O'Dell (1971) and Ney (1982), rearrangement of Equation (7.1) in the form

$$
\frac{A}{1-A}=\frac{Q_{\text {sca }}}{Q_{\text {abs }}},
$$

permits estimates of the single scattering albedo from simultaneous measurements of the scattered light (proportional to $Q_{\text {sca }}$ ) and of the thermal flux in the infrared (proportional to $Q_{\text {abs }}$ ). Ney and Merrill (1976) estimated $A$ in the range 0.3 to 0.5 from their measurements of Comet West (1975n) using this approach. Much smaller values for 
$A$ result from the geometric albedo adopted above if we use the scattering function of Figure 20 in Equation (7.2), considering that $Q_{\text {ext }}$ is expected to range between about 2 and 4 for these particles at visible wavelengths (cf. Figure 32 of van de Hulst, 1957). In this approach, for example, the values $p=0.03, \Phi_{\pi}=0.069 \mathrm{sr}^{-1}$, and $Q_{\text {ext }}=2.8$ yield $A=0.05$. Thus the effective single scattering albedo for the ensemble of dust grains is not well determined at this time.

Oishi et al. (1978) measured the polarization in the dust coma of Comet West (1975n) at wavelengths 1.0 to $2.2 \mu \mathrm{m}$ for $70<\theta_{s}<120^{\circ}$. A maximum polarization near $30 \%$ ocurred at 80 to $90^{\circ}$ for all wavelengths. The polarization was similar at visual wavelengths (Kiselev and Chernova, 1981). Negative polarization (electric vector parallel to the scattering plane) is observed in comets for large scattering angles $\left(\theta_{s}>160^{\circ}\right.$; Kiselev and Chernova, 1981; Dobrovolsky et al., 1980; Myers and Nordsieck, 1984). Giese (1980) has shown that the polarization dependence can be fitted by a mixture of irregular, micron-sized dielectric and absorbing grains. Maps of polarization in the coma show considerable structure, including regions of negative polarization (Clarke, 1971; Isobe et al., 1978). Since they were obtained with a wide bandpass, it is difficult to assess the effect of gaseous emission in these maps.

Lastly, we note that emission features (near 10 and $18 \mu \mathrm{m}$ wavelength) are commonly seen in spectra of comets within $1 \mathrm{AU}$ of the Sun. Hanner (1983) has used the size distributions discussed below (Section 7.2) to generate synthetic spectra in the thermal infrared, and concludes that these emission features can be matched most simply if the dust includes both dark, absorbing grains and dielectric, silicate grains, where the former dominate. This interpretation is supported by the thermal models of Crifo (1983), by an analysis of Halley's dust tail in 1910 (Sekanina, 1981c; cf. also Section 7.2), and by the heterogeneity of the collected particles discussed in Section 7.3.

\subsection{SizE DISTRIBUTION}

Two complementary approaches to the detrmination of size distributions for cometary dust grains utilize measurements, respectively, of the thermal emission spectrum and of the dynamics of grains in coma structures (e.g., jets and haloes; cf. Section 8), in dust tails, and in anti-tails.

The first approach is based on the expectation that dust grain temperatures are determined by the equilibrium between absorption of solar energy (mainly at visible wavelengths) and thermal emission (in the infrared). Because emission is inefficient at wavelengths large compared to the grain size (i.e., $\lambda>>a$ ), the temperature of small absorbing grains depends sensitively on their size rather than on their composition. Thus an absorbing grain of radius $a>10 \mu \mathrm{m}$ has a temperature close to that of a black body, whereas one of radius $a=0.1 \mu \mathrm{m}$ can be 300 to $400 \mathrm{~K}$ hotter (Hanner, 1983). Most comets observed in the thermal infrared ( 3 to $20 \mu \mathrm{m}$ ) show color temperatures higher than the equilibrium black body temperature, indicating that most grains have sizes less than $10 \mu \mathrm{m}$ and are composed of absorbing material, consistent with the low albedo $A_{p}$ discussed in Section 7.1. Consequently, limits to the grain size distribution can be set from the observed thermal emission spectrum without precise knowledge of the grain 
composition. The basic physical assumption on which the analysis rests is that the grains are sufficiently compact that their temperature approximates that computed for spheres. If the grains are extremely fluffy the size derived from the thermal emission may not correspond to largest diameter of the particle.

Successful fits to the observed 3 to $20 \mu \mathrm{m}$ thermal emission for several comets have been achieved using a model distribution in which the differential dust concentration (sample units $\mathrm{m}^{-4}$ ) in the coma has the form (first used in the grain dynamics studies)

$$
n=g_{0}\left(1-\frac{a_{0}}{a}\right)^{M}\left(\frac{a_{0}}{a}\right)^{N}
$$

Here the grain radius $a(\mathrm{~m})$ has the lower limit $a_{0}, g_{0}$ is a normalization constant, and the exponents $M$ and $N$ (which, respectively, control the slopes at small and large radii) lead to a peak in $n$ for radius

$$
a_{p}=\frac{M+N}{N} a_{0} .
$$

Values for $a_{0}$ in the range 0.02 to $0.2 \mu \mathrm{m}$ are compatible with the infrared data; here we adopt $a_{0}=0.1 \mu \mathrm{m}$, as suggested by the smallest units in the collected particles (Section 7.3). If we also adopt $N=3.7$ (obtained from the dynamical considerations discussed below), then the peak grain radius $a_{p}$ is well determined from model fits to the thermal infrared data because the grain temperature is a sensitive function of size. Figure 21 illustrates the results derived from such fits for several comets by Hanner (1983, 1985b), using the form of Equation (7.5). The trend toward smaller size as heliocentric distance $r$ decreases can be understood if the fragmentation of friable grains increases as the gas flux $Z$ becomes more vigorous (cf. Section 5.1).

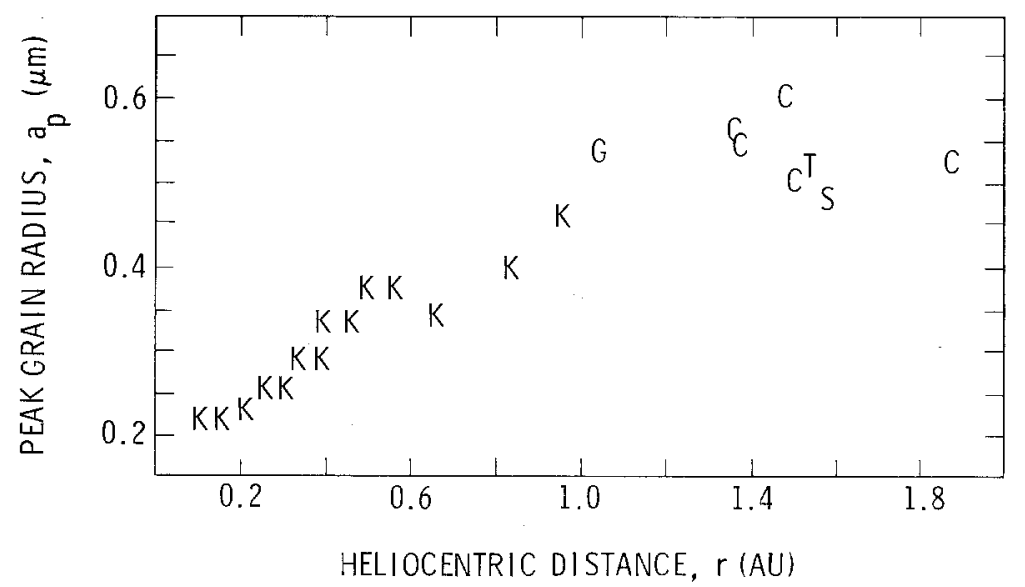

Fig. 21. Peak grain radius versus heliocentric distance for comets Kohoutek (K), $P /$ Cheryumov-Gerasimenko (C), $P /$ Grigg-Skjellerup (G), P/Tempel $1(\mathrm{~T})$, and $P /$ Stephan-Oterma (S), based on Equations (7.5) and (7.6) (using $a_{0}=0.1 \mu \mathrm{m}$ and $N=3.7$ ). 
In the dynamical approach, originated by Probstein (1969), photometric profiles in dust comae are compared to dust distributions computed from modeling of dust grain trajectories. In these analyses the natural independent variable which distinguishes among the grains is the ratio $\beta$ for the force of solar radiation pressure to that of solar gravity, given by

$$
\beta=\frac{3 L_{\odot}}{16 \pi c G M_{\odot}} \frac{Q_{\mathrm{pr}}}{\rho a} .
$$

Here the constants are identified in Table XII, and the variables include the bulk density $\rho\left(\mathrm{kg} \mathrm{m}^{-3}\right)$ and radiation pressure efficiency $Q_{\mathrm{pr}}$ for spherical dust particles of radius $a(\mathrm{~m})$. The first three rows of Table IX represent the only three cases in which the full

TABLE IX

Maximum $\beta$ from dynamical analysis of comet tails

\begin{tabular}{lll}
\hline Comet & $\beta_{\max }$ & Reference \\
\hline Arend-Roland (1957 III) & 0.55 & Finson and Probstein (1968) \\
Seki-Lines (1962 III) & 0.55 & Jambor (1973) \\
Bennett (1970 II) & 2 & Sekanina and Miller (1973) \\
$P /$ Halley (1910 II) & 2.5 & Sekanina (1983) \\
$P /$ Halley (1910 II) & 0.5 & \\
& 1.5 & Sekanina and Larson (1984) \\
Mrkos (1957 V) & 0.6 & \\
West (1976 VI) & 2.0 & Sekanina and Farrell (1982) \\
& 0.66 & \\
\hline
\end{tabular}

analysis has been completed, yielding a distribution function in $\beta$ for the dust production from the model/isophote comparison; Sekanina (1980b) summarizes the resulting distributions. Additionally Table IX lists cases for which the maximum values of $\beta$ present in the distribution have been derived. The values for $\beta_{\max }$ in Table IX correspond nicely to the maximum values for compact grains of absorbing ( $\beta_{\max }=2$ to 2.5 ) and dielectric $\left(\beta_{\max }=0.5\right.$ to 0.7$)$ materials, each having radii $a=0.1$ to $0.2 \mu \mathrm{m}$ and density close to that of solid spheres (Schwehm and Kneissel, 1981), and thus support the presence of the two grain types discussed at the end of Section 7.1.

The application of a similar dynamical analysis to comet anti-tails shows that these features (cf. Figure 25 an example), as rarely observed when the Earth is close to the comet's orbital plane, are composed of large (submillimeter) dust grains. A form similar to that of Equation (7.5), but for the differential dust production (sample units s ${ }^{-1} \mathrm{~m}^{-1}$ ), suggests that values of the exponent $N$ near 4.2 are required to fit the model to observed isophotes for several comets (Sekanina, 1979; and references cited therein). For these large particles both $\rho$ and $Q_{\mathrm{pr}}$ are approximately constant, whereas the terminal velocity $V$ is approximately proportional to $a^{-0.5}$ (cf. Section 5.2), so that the corresponding 
exponent for a concentration distribution is $N=3.7$. The fits to the thermal data discussed above (following Equation (7.6)) require $N$ in the range 3.5 to 4.6 (Hanner, 1984 b), so that a single value for $N$ connecting the characteristic size ranges for these two approaches appears reasonable. The size distribution parameters adopted for the Halley model in Appendices A and B are based on the considerations in this section.

\subsection{COMPOSITION, STRUCTURE, AND BULK DENSITY}

Because comets may well be the major source of interplanetary dust (Grün et al., 1985), we briefly describe the properties of extraterrestrial dust particles collected in the stratosphere (following Brownlee, 1978; for another review, see Fraundorf et al., 1982). The fluffy appearance of many of these particles, as illustrated in Figure 22, indeed suggests that they may be of cometary origin. About $60 \%$ of such particles are black chondritic aggregates of roughly tenth-micron sized grains with elemental abundances similar to $\mathrm{C} 1$ and $\mathrm{C} 2$ carbonaceous chondritic meteorites (although their crystal structures differ). Several percent carbon is present. The $0.1 \mu \mathrm{m}$ grains are themselves heterogeneous mineral aggregates, but they do seem to be the basic structural units and do not easily fragment into smaller components (lending support to the value for $a_{0}$ discussed in Section 7.2). About $30 \%$ of the collected micrometeorites are iron-sulfurnickel (FSN) and about $10 \%$ are mafic silicates. The FSN and silicate particles are frequently embedded in the chrondritic aggregate material. Infrared spectra of the chondritic material show a $10 \mu \mathrm{m}$ silicate feature (just as in some cometary spectra; Sanford, private communication).

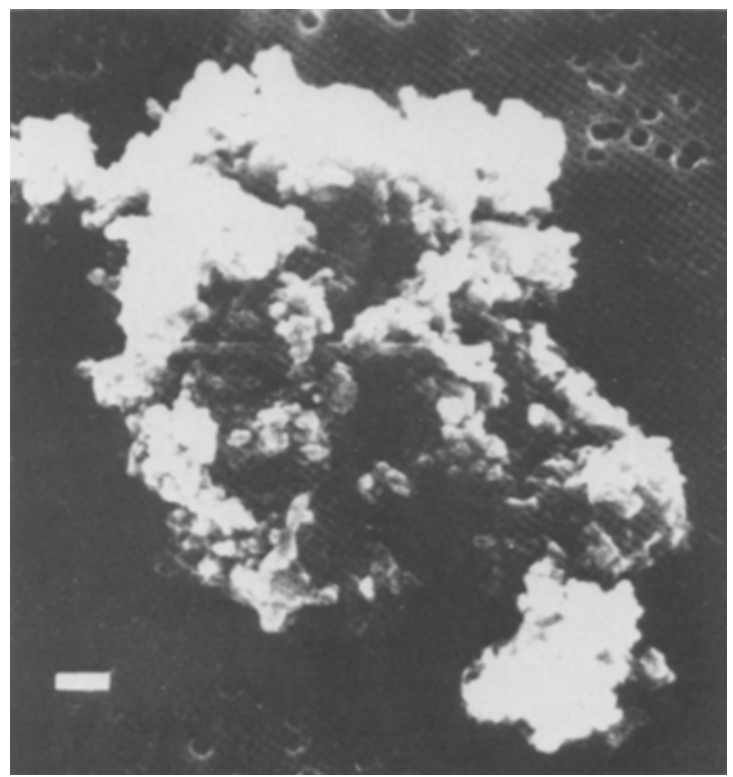

Fig. 22. Micrograph of a micrometeorite collected in the Earth's stratosphere, as an example of a possible cometary dust grain; white bar has length $1 \mu \mathrm{m}$ (from Brownlee et al., 1977). 
It has been suggested by Greenberg (1983) that dust grains recently ejected from cometary nuclei could be more complex than the Brownlee particles. If one assumes an interstellar origin of the cometary grains, one has to expect mantle-core structural building blocks. As suggested by Greenberg and sketched in Figure 23, these units
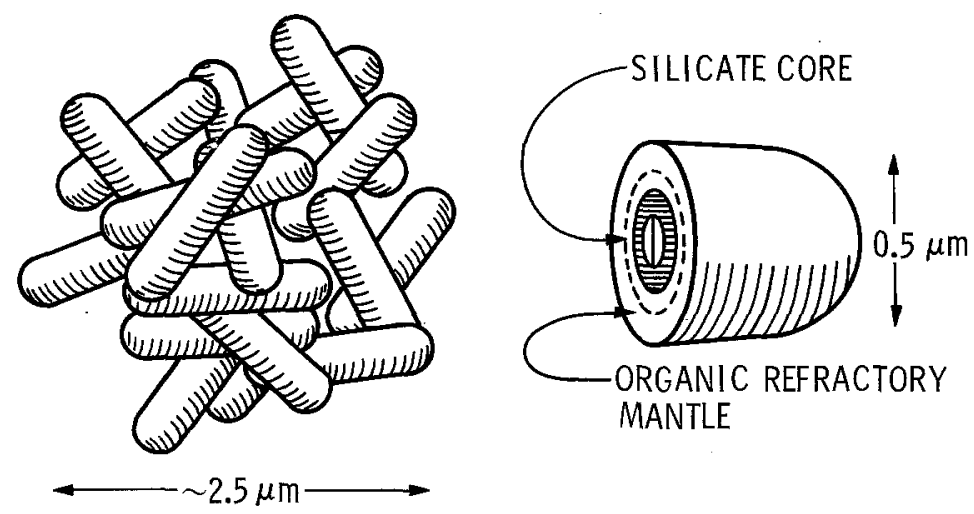

Fig. 23. Sketches of Greenberg particles (Greenberg, 1983). Left sketch shows a clump of interstellar grains, and the right sketch suggests the cross-sectional structure of an individual unit modeled after grains around protostellar objects.

consist of silicate cores surrounded by organic mantles. Compared to the Brownlee particles, these Greenberg particles might have even lower densities because the mantle material density is near $1 \mathrm{~g} \mathrm{~cm}^{-3}$, while the building blocks average $2 \mathrm{~g} \mathrm{~cm}^{-3}$ (including the core density of $3 \mathrm{~g} \mathrm{~cm}^{-3}$ ). As discussed by Johnson et al. (1984), the mantle material, when exposed to sputtering by the solar wind, becomes black because sputtering of organic material leaves behind more carbon than other light elements. Mukai and Fechtig (1983) have discussed the possible disappearance of the organic mantles with time due to sputtering effects and to high temperature sublimation during perihelion passages. Since the sputtering rates are much larger from the organic material than from silicates and because of asymmetric sublimation, the silicate cores finally survive, with the result that the Brownlee particles could possibly evolve from Greenberg particles.

The chemistry of cometary dust is thought to be extremely primitive. It could possibly be related to the carbonaceous chondrites which are the most primitive meteoritic material containing a comparatively high fraction of volatiles. The major building blocks of the Brownlee particles resemble those of individual grains in carbonaceous chondrites, as listed in Table X (for further information, see Dodd, 1981). If one accepts the relation of cometary grains with carbonaceous chondrites one should note that the latter also contain organic material. To what degree this organic material is representative of the organic mantle material of the Greenberg particles is unknown. Greenberg (1983) has simulated cometary mantle material using a sequence of photoprocessed reactions of the light elements $\mathrm{H}, \mathrm{C}, \mathrm{N}$, and $\mathrm{O}$. The result is a rather complex yellow organic material. 
TABLE X

Major mineral components of carbonaceous chondrites

\begin{tabular}{ll}
\hline Clinopyroxene & $(\mathrm{Mg}, \mathrm{Fe}, \mathrm{Ca}) \mathrm{SiO}_{3}$ \\
Gypsum & $\mathrm{CaSO}_{4} \cdot 2 \mathrm{H}_{2} \mathrm{O}$ \\
Kamacite & $\mathrm{Fe}_{0.93} \ldots 0.96 \mathrm{Ni}_{0.07} \ldots 0.04$ \\
Magnetite & $\mathrm{Fe}_{3} \mathrm{O}_{4}$ \\
Melilite & $\mathrm{Ca}_{2} \mathrm{Al}_{2} \mathrm{SiO}_{7}$ \\
Montmorillonite & $\left(\frac{1}{2} \mathrm{Ca}, \mathrm{Na}_{0.7}(\mathrm{Al}, \mathrm{Mg}, \mathrm{Fe})_{4}(\mathrm{Si}, \mathrm{Al})_{8} \mathrm{O}_{20}(\mathrm{OH})_{4} \cdot \mathrm{nH}_{2} \mathrm{O}\right.$ \\
Olivine & $(\mathrm{Mg}, \mathrm{Fe})_{2} \mathrm{SiO}_{4}$ \\
Organics & $($ mostly aromatic polymers $)$ \\
Orthopyroxene & $(\mathrm{Mg}, \mathrm{Fe}) \mathrm{SiO}_{3}$ \\
Plagioclase & $\mathrm{Na}, \mathrm{Al}, \mathrm{Si}_{3} \mathrm{O}_{8} \ldots \mathrm{Ca}, \mathrm{Al}_{2}, \mathrm{Si}_{2}, \mathrm{O}_{8}$ \\
Serpentine & $\left(\mathrm{Fe}+, \mathrm{Mg}_{6}\left(\mathrm{Si}, \mathrm{Al}, \mathrm{Fe}^{3+} \mathrm{O}_{10}\right)(\mathrm{OH})_{8}\right.$ \\
Spinel & $\mathrm{MgAl}_{2} \mathrm{O}_{4}$ \\
Troilite & $\mathrm{FeS}$ \\
Water & $\mathrm{H}_{2} \mathrm{O}$ \\
\hline
\end{tabular}

The structure of the collected (Brownlee) particles implies that the smallest grains of radius near $0.1 \mu \mathrm{m}$ have densities close to the material bulk density (consistent with $\beta_{\max }$ in Table IX) whereas larger particles (which are aggregates of these small units) can have onsiderably lower densities. Brownlee (1978) has estimated densities near 1 to $3 \mathrm{~g} \mathrm{~cm}^{-3}$ based on composition and physical appearance for particles in the size range 4 to $40 \mu \mathrm{m}$.

The depth-to-diameter ratio of lunar microcraters depends on the density of the impacting interplanetary dust particle. On this basis Brownlee et al. (1975) obtained a density of 2 to $4 \mathrm{~g} \mathrm{~cm}^{-3}$, while Nagel et al. (1976) found evidence for three components corresponding to iron, silicates, and something with density near $1 \mathrm{~g} \mathrm{~cm}^{-3}$. Extremely low-density grains, however, may not have created observable microcraters. Grün et al. (1980) interpret data from the HELIOS micrometeoroid experiment as indicating that 10 to $20 \%$ of the particles have density less than $1 \mathrm{~g} \mathrm{~cm}^{-3}$. Mukai and Fechtig (1983) have suggested that cometary particles are originally even more porous agglomerates. As the volatiles sublime, the solid grains become more closely packed so that particle density increases with time.

Studies of meteor streams identified with comets show that these larger cometary dust grains can have extremely low densities. Millman (1976) tabulates mean densities ranging from near $1 \mathrm{~g} \mathrm{~cm}^{-3}$ for the Geminids to less than $0.01 \mathrm{~g} \mathrm{~cm}^{-3}$ for the Giacobinids, while Verniani (1973) found values near $0.8 \mathrm{~g} \mathrm{~cm}^{-3}$ for radio meteors.

This diverse evidence suggests that a particle density $\rho$ which varies with grain radius $a$ could be useful for modeling purposes. Thus we have proposed the form

$$
\rho=\rho_{0}-\sigma_{0}\left(\frac{a}{a+a_{2}}\right)
$$

and the parameter values $\rho_{0}=3 \mathrm{~g} \mathrm{~cm}^{-3}, \sigma_{0}=2.2 \mathrm{~g} \mathrm{~cm}^{-3}$, and $a_{2}=2 \mu \mathrm{m}$. The resulting densities range from 3.0 to $0.8 \mathrm{~g} \mathrm{~cm}^{-3}$ as the particle size varies from the smallest to the largest in the distributions. 
Lastly we note that it is unlikely that icy grains will be present in Halley's coma within $2 \mathrm{AU}$ of the Sun. Water-ice particles can be lifted off the surface only by an outflowing gas which sublimates from ices more volatile than $\mathrm{H}_{2} \mathrm{O}$, so that a halo of water-ice grains would be expected only at larger heliocentric distances (Mendis et al., 1985). Indeed they have been identified only in two comets, both beyond $3 \mathrm{AU}$ (Campins et al., 1983; and Hanner, 1984a). Although pure water ice particles (or pure ice mixed with pure silicate) can survive for years at $r=1 \mathrm{AU}$ (Patashnick and Rupprecht, 1977), the slightest admixture of dark, absorbing material reduces the lifetime to hours or minutes and the range to a few hundred $\mathrm{km}$ (Hanner, 1981). This conclusion is consistent with the low albedos discussed in Section 7.1.

\subsection{Dust CONCENTRATION IN THE COMA AND TAIL}

Outside the region of collisional acceleration (Section 5.2) each dust particle moves independently along a ballistic trajectory responding to the forces of solar gravitation and radiation pressure; those of cometary and planetary gravitation are negligible for present purposes. For most dust particles the volume limited by cometocentric distances $300<R<3000 \mathrm{~km}$ is effectively force-free, so that the ballistic trajectories can be approximated by simple radial outflow at constant speed $V$, and the dust concentration $n$ is proportional to $R^{-2} V^{-1}$.

Because the solar gravitation and radiation pressure forces are both radial and proportional to $r^{-2}$ (where $r$ is heliocentric distance), the dust particle trajectories obey Keplerian orbital mechanics with an effective central force constant $(1-\beta) G M_{\odot}$ (sample units $\mathrm{m}^{3} \mathrm{~s}^{-2}$ ), where the ratio $\beta$ of the two forces is given by Equation (7.7). For short times of flight from the nucleus, which lead to trajectory segments which are small fractions of the heliocentric distance $r$, we consider the acceleration of the dust in a cometocentric bipolar system (CBS, with the nucleus at the origin, and the positive $S$-axis along the prolonged heliocentric radius vector). The acceleration can be well approximated by a vector having only a constant $S$-component, such that a 'fountain model' is appropriate for dust particles which leave the nucleus with various terminal speeds $V$ and directions. In CBS coordinates the particle trajectories are parabolic, each parabola has one point tangent to an envelope specific to the values of $V$ and $\beta$, and the envelope is a paraboloid of revolution with focus at the nucleus. As illustrated in Figure 24, the axis of symmetry is the Sun-comet line ( $\pm S$-axis), and the apex is sunward of the nucleus by the distance

$$
E=\frac{r^{2} V^{2}}{2 \beta G M_{\odot}}
$$

Typical apex distances increase from $E \simeq 10^{4} \mathrm{~km}$ for micron-size particles to $E \simeq 10^{5} \mathrm{~km}$ in a broad maximum for millimeter-size particles, for an active comet near $r=1 \mathrm{AU}$.

The fountain model predicts that at a given location (specified in CBS by $S$ and by cometocentric distance $R$ ), there are zero, one or two solutions for dust trajectories from the nucleus, corresponding to locations beyond, on or within the envelope, respectively. 


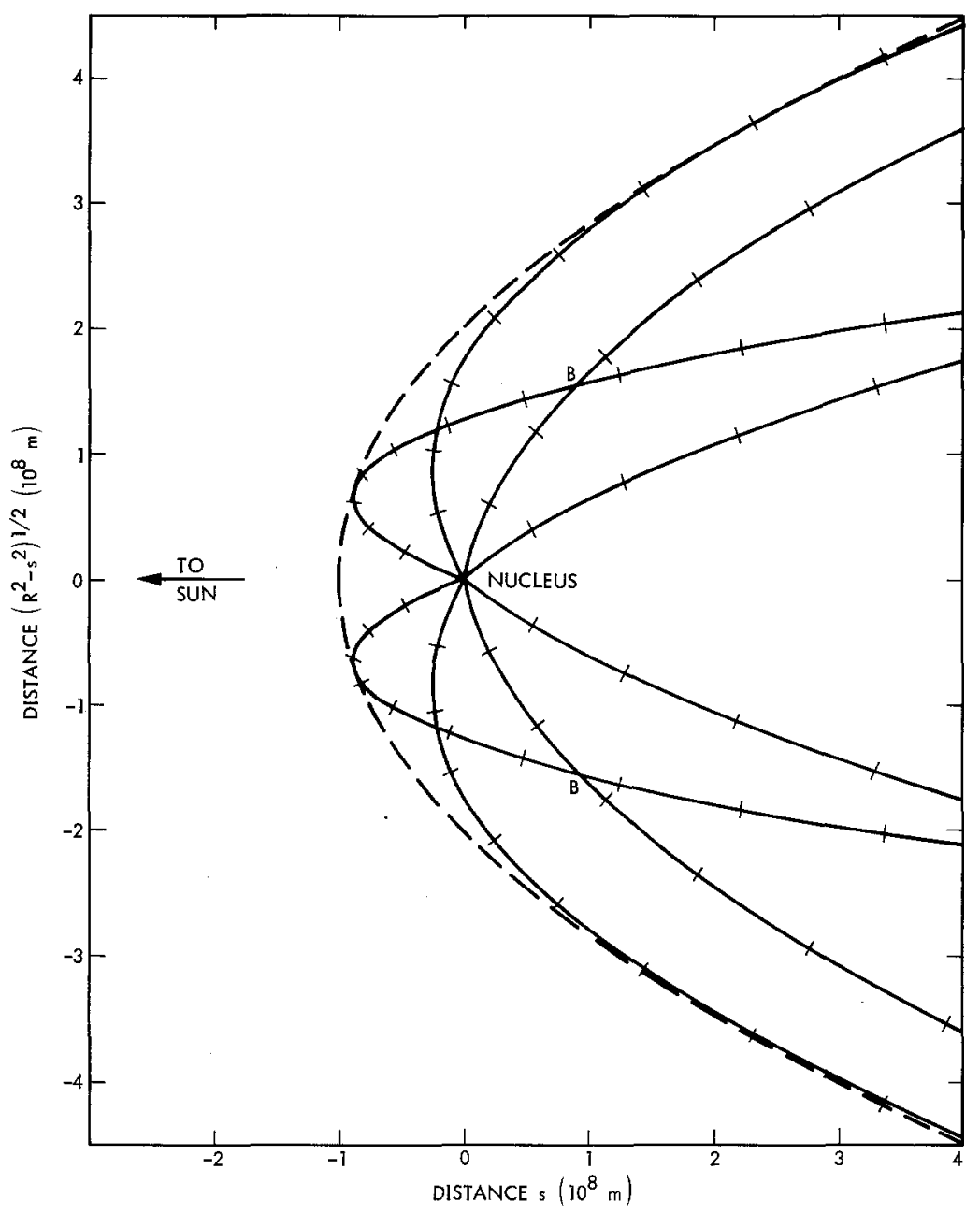

Fig. 24. Fountain model trajectories (solid lines) for dust particles having $\beta=0.08$ and $V=347 \mathrm{~m} \mathrm{~s}^{-1}$ at $r=0.89 \mathrm{AU}$, wxith time ticks every two days. At points $B$, direct (reflected) particles are present which were emitted from the nucleus $5.3(15.2)$ days earlier at an angle $100^{\circ}\left(20^{\circ}\right)$ from the subsolar direction. The envelope, with apex distance $E=10^{8} \mathrm{~m}$ for these particles with mass near $10^{-12} \mathrm{~kg}$, is shown dashed.

For the last of these cases the real parameter

$$
\eta=\frac{R}{(2 E+S)+\left[(2 E+S)^{2}-R^{2}\right]^{1 / 2}},
$$

permits the specification of times of flight from the nucleus as

$$
\left(t-t_{e}\right)=\left(4 E R \eta^{ \pm 1}\right)^{1 / 2} / V,
$$

for particles which are on trajectories 'direct' $\left(\eta^{+1}\right)$ from the nucleus, or which have been 'reflected' $\left(\eta^{-1}\right)$ at the envelope, as illustrated in Figure 24. To describe the emission 
of dust particles from the nucleus (now considered as a point source), we introduce the directional distribution $f_{s}\left(\mathrm{sr}^{-1}\right)$ and the differential production $q\left(\mathrm{~s}^{-1} \mathrm{size}^{-1}\right)$. The latter is proportional to the product of the ratio of total dust-to-gas production by mass, the gas production rate $Q_{g}$ (both discussed in Section 3.2), the gas mean molecular mass $M_{g}$, and the dust source size distribution function $g_{a}$, in the form

$$
q=\frac{\mu M_{g} Q_{g} g_{a}}{\left[\int_{a_{0}}^{a_{3}} \mathrm{~d} a \frac{4 \pi}{3} \rho a^{3} g_{a}\right]\left[\int_{4 \pi} \mathrm{d} \Omega f_{s}\right]}
$$

Here it is convenient to include normalization constants in both $g_{a}$ and $f_{s}$ so that each of the two integrals in the denominator of Equation (7.12) has the value unity. The dust spatial concentration $n\left(\mathrm{~m}^{-3}\right.$ size $\left.^{-1}\right)$ is then given by

$$
n=q f_{s}\left|\frac{\sin \theta_{e}}{J_{c}}\right|
$$

where the Jacobian $\left(\mathrm{m}^{3} \mathrm{~s}^{-1} \mathrm{sr}^{-1}\right)$ takes the two forms

$$
J_{c}=\frac{\partial(x, y, z)}{\partial\left(\theta_{e}, \phi_{e}, t_{e}\right)}=\left\{\begin{array}{l}
R^{2} V\left(\eta^{2}-1\right), \\
R^{2} V\left(\eta^{-2}-1\right),
\end{array}\right.
$$

for direct and reflected particles, respectively. Here $X, Y$, and $Z$ represent any set of Cartesian coordinates of the target point at which $J_{c}$ and $n$ are required, and the variables $q, f_{s}$, and $V$ are evaluated for the emission direction $\theta_{e}$ and $\phi_{e}$, at time $t_{e}$.

For times of flight exceeding several days a more complete ballistic theory which correctly handles the Keplerian heliocentric trajectories (from nucleus to target) for the dust particles is required. Comprehension of the relevant dynamics is aided by the construction, for particles of negligible emission velocity $V$, of synchrones, i.e., loci of particles emitted at a single time with various values of $\beta$, and of syndynes, i.e., loci of particles all having the same $\beta$ emitted at different times. In Figure 25 these loci are shown to illustrate the distortions caused both by the varying differential acceleration of the nucleus and the dust (which seems to be swept to regions exterior to and lagging the motion of the nucleus) and by the projection from the orbit plane to that of the sky.

Digital computers enable the numerical realization of such trajectories for arbitrary cometary motion and dust particle properties, and permit the evaluation of the Jacobian appropriate for use in Equation (7.13) when the full theory is used (and the first of the two equalities in Equation (7.14) is maintained). In this case iterative procedures are required to connect the emission and target points (e.g., along a spacecraft trajectory near a comet) for dust particles having known variations of $\beta$ and $V$. Appendix $A$ presents parameter values and assumptions for one set of extensive calculations intended as a Halley dust model, and sample results are presented in Appendix B. In these calculations the size distribution function for the dust production $g_{a}\left(\mathrm{~kg}^{-1} \mathrm{~m}^{-1}\right.$; 

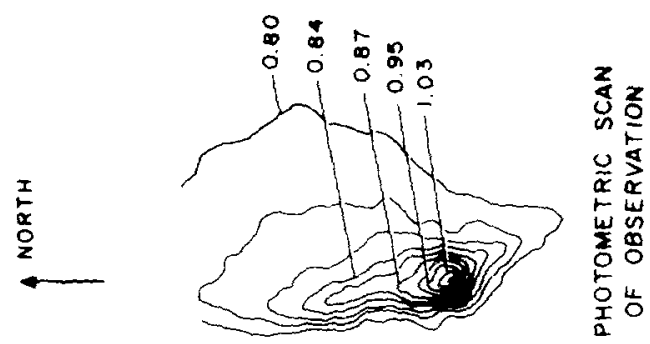

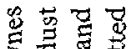

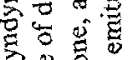

के

要要要

政密

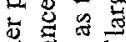

诺 है

㱐

密

氙

g

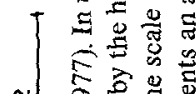

$\because$

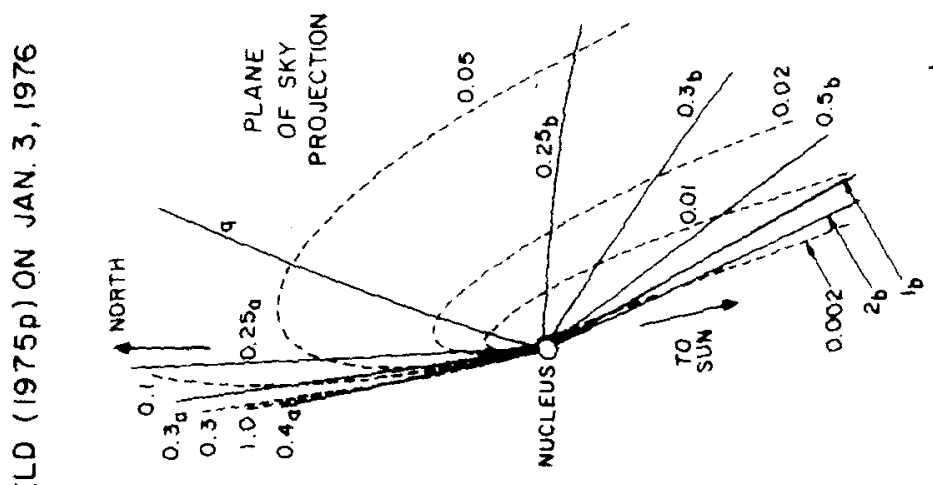

言兔完

政

送尊

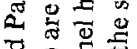

敢

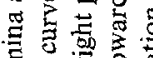
踏

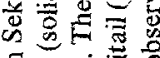
영

西

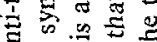

ब.

")

$\approx$

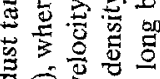

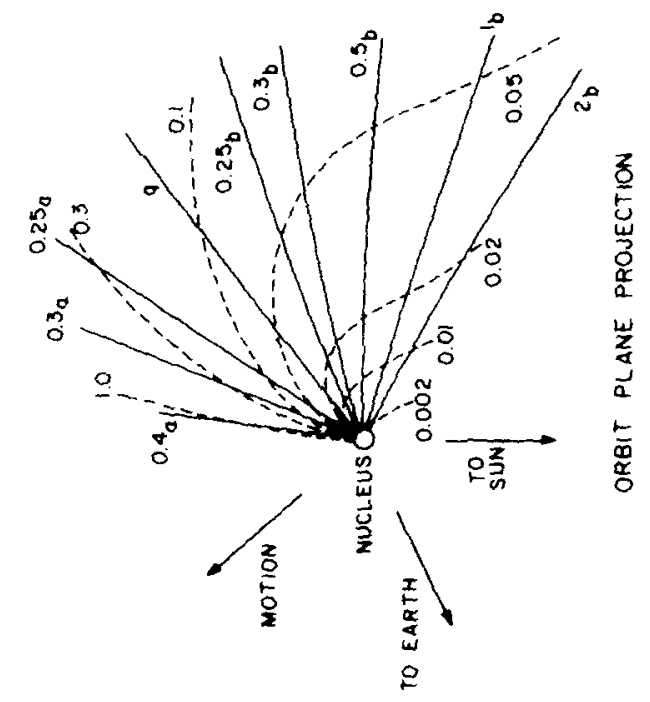

를

旁

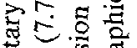

동

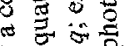

웜

吸

s.

용

สำ

过

要施

올

额要

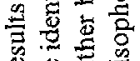

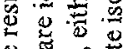

응

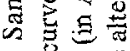

的总密

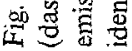


cf. Equation (7.12)) has the form given by the right-hand side of Equation (7.5), and the directional distribution of the dust production

$$
f_{s}=A_{0}-A_{3} \cos \vartheta,
$$

varies smoothly with angular distance $\vartheta$ from the anti-Sun direction. These distributions, and the dependence of $V$ on particle size, are identical for the two dust kinds discussed at the end of Section 7.1, but the dependence of $Q_{\mathrm{pr}}$ and $\beta$ (Equation (7.7)) on particle size differs for the absorbing and dielectric grains (see Table XVI).

At the scales employed in astronomical photography of dust features in comet comae and tails, the full orbital theory is often required for useful analysis. The features to be discussed in Section 8 are not dust particle envelopes, synchrones, nor syndynes, but rather are loci of particles emitted from discrete locations on the nucleus' surface during discrete time intervals, e.g., active areas during successive rotations. The signatures of the dynamics involved are sufficiently unique to allow the identification of several parameters of the dust emission responsible for the photographed features, and the analysis contrasts markedly with the homogeneous and continuous description presented above.

\section{Cometary Active Regions}

Many brighter comets, including Comet $P /$ Halley, show coma structure that changes from night to night. The features, which include jets, fans, arcs, and halos, are products of directed emission of material and serve as evidence of a nonuniform surface structure of the cometary nucleus. Visual telescopic observations emphasize the boundaries of changing intensity gradients and make the usually low-contrast coma features look more prominent than they in fact are. In general, the visibility of these features depends upon aperture, magnification, and also sky brightness and transparency. Together with varying competence of the visual observers these conditions are commonly responsible for an embarrassing lack of resemblance among drawings obtained at virtually the same time. With rare exceptions, quantitative analysis of drawings is not possible. The following sections describe the properties of active regions as derived for Halley from 1910 photographs.

\subsection{Photographs of halley and their image PRocessing}

Photographic observations of coma structure are free from many pitfalls of visual observation, but have problems of their own. A useful photograph must have a high spatial resolution, a correct exposure time, precise guiding on the comet, and must be obtained in good seeing. The choices of emulsion and filter as well as the photographic processing also affect the quality of the result. Finally, a successful positional reduction and analysis require that the features be visible in the eyepiece of the measuring machine under magnification that reduces the contrast. Only recently has this last condition been satisfactorily accommodated by the digital image processing of the photographs and the 
situation further improved by the introduction, at the telescope, of highly sensitive solid-state detector arrays, such as charge-coupled devices.

Only a limited number of high-resolution photographs is available from Comet Halley's 1910 apparition. At the time of this writing, processing of selected plates obtained at the Mount Wilson, Helwan, Lick, and Lowell Observatories has been completed. Analysis of the comet's images on these photographs as well as on selected photographs taken elsewhere (Cordoba, Argentina; Heidelberg, Germany; and Vienna, Austria) is near completion. The following is a progress report that summarizes the results that have been published to date (Larson and Sekanina, 1984, 1985; Sekanina and Larson, 1983, 1984, 1986).

Since the features in Comet Halley's atmosphere are of low contrast and superimposed on an intensity gradient of long dynamic range, use of digital image-processing techniques is essential for enhancing the contrast. Various approaches to image enhancement have been applied to comets. The most common techniques utilize spatial filtering or take intensity derivatives in some direction, thereby reducing the effects of the steep intensity fall-off. One of the most successful methods has been the linear intensity derivative using a linear shift-difference algorithm. The primary limitation of this technique is that it emphasizes only the features which have an intensity change in the direction of the shift.

One characteristic property of $P / \mathrm{Halley}$ is the persistent appearance of nearly-circular halos, centered approximately on the nucleus. It appears that a shift difference radial to the center of light should improve visibility of these features, as it maps the rate of change of emission at a given position angle. A severe limitation is that the features oriented radial to the nucleus, such as jets or ion streamers, do not show. To make these features visible, a rotational shift difference (about the center of light) must be applied. The rotational component tends to emphasize features of varying spatial extent depending upon the distance from the nucleus. This, however, is not a serious problem as features become larger and more diffuse farther from the nucleus and require a larger shift to show.

To ensure that features of any orientation are retained in the processing, two images which have the same radial shifts and rotational shifts of the same magnitude but opposite directions are added. The optimum shift parameters must be found empirically in each case. It is important to consult the original image to ensure that processing artifacts are not introduced and that the general appearance of the features is not distorted so as to mislead interpretation. The final image includes significantly enhanced emission boundaries, which greatly facilitate analysis of the evolution of dust features.

\subsection{DESCRIPTION OF FEATURES AND THEIR SOURCES ON THE SURFACE}

A study of the coma morphology is complicated by the broad spectral bandpass of the emulsions used, with the contributions from the usual molecular species, from scattered solar continuum, and from ion emissions. Identification of these components in the direct images can only be made using guidelines based on their recognized morphological characteristics in comets. We identify solids by their sunward asymmetry and by 


\section{COMET HALLEY 1910 II}
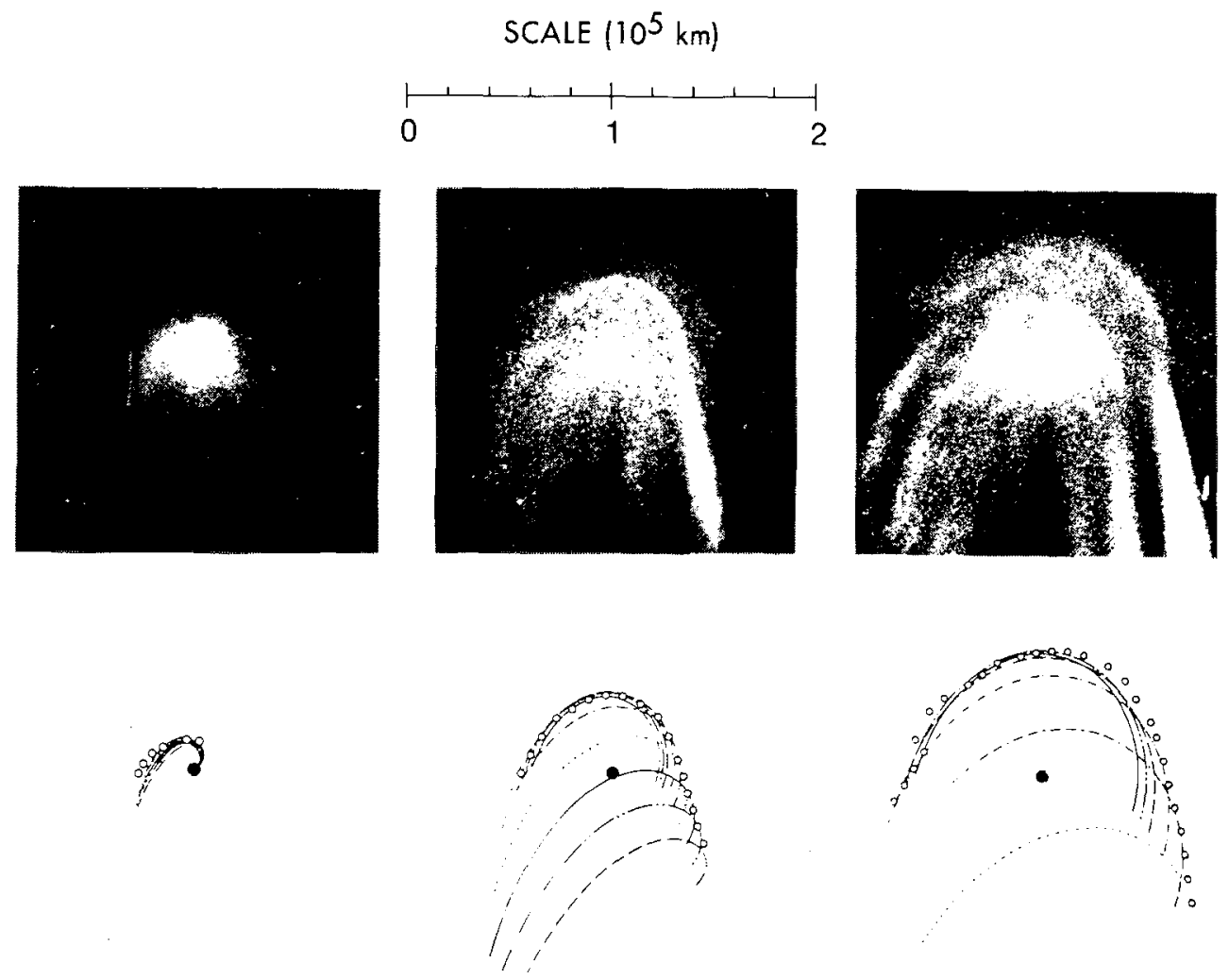

7.4931

8.4885

9.4875

\section{MAY (UT)}

Fig. 26. Upper row: Digitally processed images of Comet Halley on the Mount Wilson plates taken on 1910 May 7-9 (Sekanina and Larson, 1984). The near-nucleus spiral jet on May 7 develops into the expanding halo or envelope on May 8-9. Images are oriented with the Sun at the top. Original photographs courtesy of the Mount Wilson and Las Campanas Observatories. Lower row: Comparison with the model of continuous dust ejection from a discrete emission region on the rotating nucleus' surface. A perfect match between theory and measurement is achieved when the measured points (open circles) lie precisely on the envelope of the several particle loci. Each such curve represents particles ejected with a particular initial velocity and subjected to a particular radiation-pressure acceleration. Times of observation are in UT.

changes that imply subkilometer-per-second velocities. Because of chaotic molecular motions, gas emissions have a nearly symmetric distribution and lack discrete features. Ion emissions are identified by sharply defined structure which changes on very short time scales as a result of the interaction with the highly variable solar-wind magnetic field. 
The single most striking quality of the processed images, as in Figure 26, for example, is evidence of the constant formation of spiral jets that 'unwind' from the nucleus condensation on the sunward side of the comet's head and develop into rapidly expanding and usually somewhat asymmetric envelopes. Sekanina and Larson's (1984) detailed analysis demonstrates that this pattern is diagnostic of continuous emission of dust from discrete sources on the sunlit hemisphere of a rotating nucleus.

Assuming that the nucleus equatorial plane coincides with the orbit plane, consider the evolution of a dust feature made by continuous emission from an equatorial point-like source of particles that are being ejected from sunrise till sunset with a constant velocity and exposed to a constant pressure from solar radiation. In Figure 27 this evolution is compared for two comets of very unequal spin rates, as viewed pole-on. The differences are striking. In the case of a rapidly rotating comet $(P<<1$ day) the feature separated from the nucleus so early that it is virtually impossible to observe the spiral-jet phase of development, unless the comet makes a close approach to the Earth. Within a few rotations after the beginning of emission, the feature evolves into an envelope of fairly symmetrical, almost semi-circular shape approximately concentric with the nucleus and of large enough dimensions to be easily detected by a ground-based

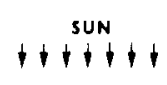

ROTATION PERIOD $=4.8 \mathrm{HR}$
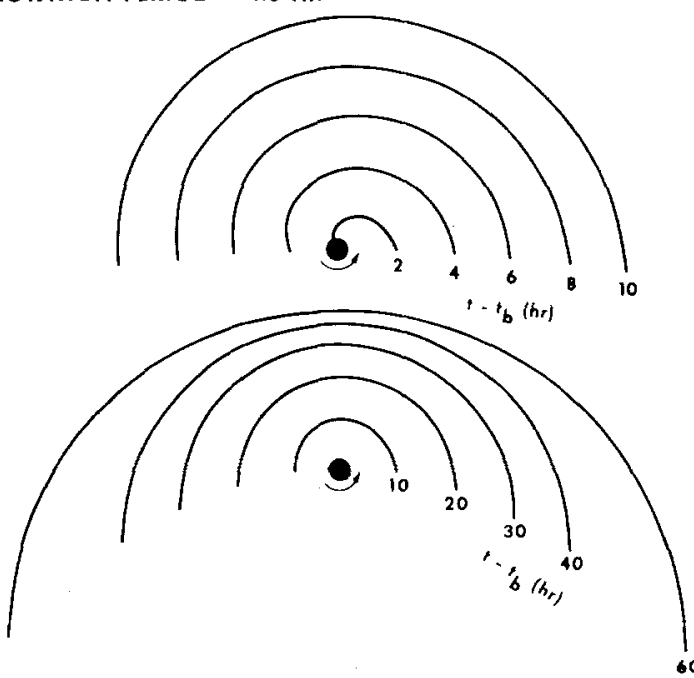

SUN

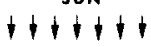

ROTAIION PERIOD = $48 \mathrm{HR}$

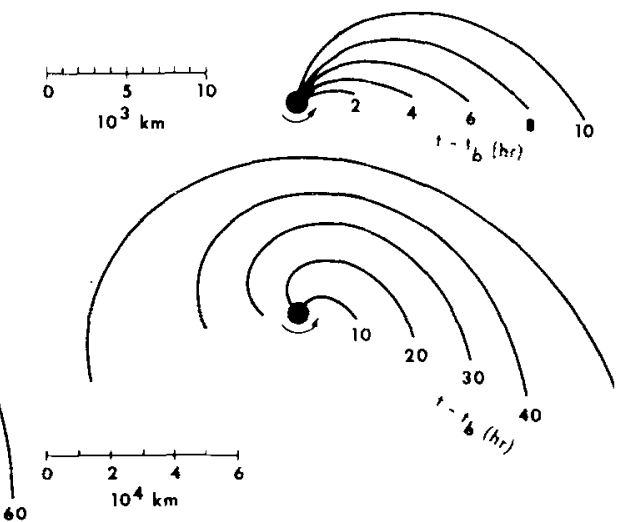

Fig. 27. The effect of the rotation period on the evolution of dust ejecta from a point-like source on the equator of a spinning nucleus (Sekanina and Larson, 1984). It is assumed that the spin axis is normal to the comet's orbit plane, which lies in the plane of the figure; that the source is active from sunrise to sunset; and that all particles are ejected with the same velocity of $500 \mathrm{~m} \mathrm{~s}^{-1}$ and exposed to a constant solar radiation-pressure field with a repulsive acceleration of $0.25 \mathrm{~cm} \mathrm{~s}^{-2}$. The circles show the positions of the nucleus, while the curves describe the loci of ejected particles at various times after the beginning of emission. The sense of rotation and the direction to the Sun are also indicated. The left-hand side of the figure depicts the evolution of ejecta from a rapidly rotating nucleus; the right-hand side, from a slowly rotating nucleus. Note the different scales for the early (top) and late (bottom) phases of development. 
observer. As it keeps expanding nonuniformly, the envelope eventually acquires a quasi-parabolic shape. In contrast, dust particles of the same dynamical properties emitted from a slowly rotating nucleus ( $P>1$ day) form first a spiral jet, which gradually evolves into an asymmetrical envelope, only remotely resembling a skewed parabola.

The appearance of actual dust features is unfortunately much more complex because a comet's spin axis is seldom normal to its orbit plane; an active region is usually not on the equator and often may not be approximated by a point source; and, most certainly, dust particles are not all ejected with the same velocity and subjected to the same radiation-pressure acceleration. Also, the Earth's cometocentric position is seldom anywhere near the direction of the comet's spin axis, so that features projected onto the plane of the sky are foreshortened by some unknown amount.

In the early phase of continuous emission of dust from a small discrete region on the sunlit hemisphere of a rotating cometary nucleus, the ejecta always are essentially a two-dimensional formation expanding along a conical surface with the vertex angle equal to twice the co-latitude of the emission region. With increasing age, emitted dust particles are under progressively increasing effects of radiation pressure and the ejecta's initially conical surface is rapidly distorted. This model of formation of the envelopes is contrary to a traditional view which regards envelopes as projected paraboloids of isotropic emission (cf. Section 7.4). To avoid any misunderstanding, it is emphasized that this is not to say that dust ejected isotropically should not be confined to a volume about the nucleus approximately circumscribed by a paraboloid-shaped envelope as in the fountain model. Rather, this $i$ s to say that the observed envelopes have nothing in common with isotropic emission and the fountain model. Indeed, the unacceptably large differences between the properties of Halley's envelopes and the predicted properties for the paraboloid envelopes (especially the deviations from the expected $2: 1$ ratio of the semilatus rectum to the vertex distance), discussed by Bobrovnikoff (1931), provide sufficient evidence to dismiss the traditional approach.

\subsection{MODELING THE EVOLUTION OF DUST FEATURES FOR HALLEY}

Sekanina and Larson's $(1984,1986)$ modeling of the dynamical evolution of dust features in the head of Comet Halley involves the determination of the spin vector (cf. Section 4.3) as a byproduct. Each emission region is characterized by a set of cometocentric coordinates, latitude $\phi$ and longitude $\lambda$. The latitude is measured along a meridian from the nucleus equatorial plane, positive to the northern pole (from which the nucleus is seen to rotate counterclockwise). The longitude is measured along the equator from the meridian of the subsolar point at perihelion, positive, as on the Earth, to the west. The ejection angle $\theta$ at a time $t$, equal numerically to the Sun's hour angle, is related to the longitude by

$$
\lambda=\frac{2 \pi}{P}(t-T)+\theta_{0}(T)-\left[\theta(t)+\theta_{0}(t)\right] .
$$

Here $P$ is the sidereal rotation period, $T$ is the time of perihelion passage, $\theta_{0}$ the longitude 
difference between the comet's vernal equinox and the subsolar point,

$$
\tan \theta_{0}(t)=(\cos I) \tan (\Phi+u),
$$

$\Phi$ and $I$ are the spin-axis parameters (Section 4.3), and $u$ is the comet's true anomaly at time $t$ (cf. Figure 5 and Table IV).

The dependence of the evolution of a dust-emission feature on the dynamical behavior of the ejecta is most directly expressed by the parametric function that relates particle ejection velocity $V$ to the dimensionless acceleration $\beta$ by solar radiation pressure (Equation (7.7)). Feature boundaries may also depend on the times (or angles) of emission beginning and/or termination in each rotation. An elaborate model may consider variations in the ejection velocity with the Sun's elevation above the local horizon and, if the photometric profile of the feature is known, the particle mass distribution and the relation ship between mass and $\beta$, which involves the particles' mass, density, shape, and radiation pressure efficiency. In the first approximation, however, it can be assumed that ejection velocity is a unique function of $\beta$ for each feature, independent of the Sun's elevation angle and particle type.

Particles with different values of $\beta$ and $V$ ejected during one rotation line up, at any specific time after the beginning of emission, along various curves of the kind illustrated in Figure 27. These loci have a common envelope which, in projection onto the plane of the sky, defines the calculated halo dimensions confining the ejecta. The objective is to find, by trial and error, the cometocentric coordinates of the site of each dust-emission source on the nucleus surface and to derive the parametric function which relates $\beta$ and $V$, so that the envelope of the particle loci approximates the feature's observed boundary as closely as possible. A check on each such solution is provided by fitting the positions of the feature's boundary on more than one day.

A feature's expansion rate, hence its growth from day to day, is a direct measure of the parametric function. From theory it follows that the particles ejected with higher velocities are, as a rule, also subjected to higher radiation-pressure accelerations and vice versa. This results in a systematic decrease with time in the value of $\beta$ (and, therefore, typically in an increase in the mass) of particles that are responsible for the feature's observed boundary. In the early phase of development the boundary is made of particles ejected with the highest velocities, but the heavier particles soon become dominant, as it takes longer for them to be driven tailward by radiation pressure. Also, ejecta of different $\beta$ and $V$ contribute unevenly to different portions of the halo profile and boundary.

The analysis is illustrated using the feature of 1910 May 7-9. The observed development of this spiral jet into an envelope is shown in Figure 26 (upper panels). The extent of the May 7 spiral is readily apparent on the processed image, but is difficult to follow on the north because of its proximity to the nucleus condensation. The photograph taken the following day (May 8) shows the spiral evolved into a well-defined envelope with an apparent latus rectum-to-vertex distance ratio of about $2: 1$ (parabola has a ratio of $4: 1$ ). The sunward and northern edges appear particularly sharp. By May 9, this envelope had become larger and much more diffuse, but the general outline shape of the previous day had been maintained. 
The feature's measured boundaries on May 7-9 are compared with the model in Figure 26 (lower panels). Each of the curves represents a locus of particles which are ejected from the region between sunrise and sunset and which are characterized by a pair of specific values of $\beta$ and $V$. The quality of fit is evident from the degree of correspondence between the boundary measurements and the common envelope to the particle loci.

The beginning of ejection from the region, essentially at sunrise, is dictated by the extent to the south of the May 7 spiral jet and the May 8 envelope. Without detailed photometry of the feature, one cannot establish the time the activity ceased. From the width of the envelope, ejection must have proceeded for more than 0.6 day. The ejection velocities range from $350 \mathrm{~m} \mathrm{~s}^{-1}$ to almost $700 \mathrm{~m} \mathrm{~s}^{-1}$; the particles observed vary generally from submicron-sized to about $10 \mu \mathrm{m}$ in radius. Still larger particles should be responsible for boundaries in very late phases of feature evolution, when the surface brightness drops below the plate detection limit, due apparently to the small cross section-to-mass ratio of such very large particles.

\subsection{ENHANCEMENT OF DUST CONCENTRATION IN JETS}

A large number of dust jets, each observed on at least three consecutive dates, have been successfully modeled from 1910 photographs of Halley. Interpretation of the resulting ejection-velocity function in terms of Probstein's (1969) formalism of interaction between dust and gas in cometary atmospheres allows one to estimate the production rates of gas and dust per unit surface area of an emission region and to compare them with the average production rates of gas and dust per unit area of the comet's surface (cf. Sections 5 and 7.4). The results suggest that, in general, the dust flux from an active region exceeds the flux from the surrounding surface much more substantially than does the gas flux. It appears that several discrete regions that are active simultaneously may account for a significant fraction of the instantaneous rate of dust injection into the comet's atmosphere, even though they may represent only a few percent of the nucleus surface.

The results of these calcultions seem to agree in essence with the results of Larson and Sekanina's (1984) photometric study of a bright jet from two plates taken on 1910 May 9 (Figure 28). Since the plate emulsion used was sensitive to the major molecular emissions $\left(\mathrm{C}_{2}\right.$ and $\left.\mathrm{CN}\right)$ as well as a broad range of the continuous spectrum, the molecular and dust components must be deconvolved in order to estimate the ratio of scattered light in and outside the jet, the quantity related to the excess dust-particle number density in the jet as compared to the average density in the surrounding coma. Because collisions rapidly lead to random molecular motions, gas emissions from the various regions of the nucleus have a tendency to blend indiscriminately in the coma, so that the column densities of a neutral-gas species measured in the jet and in the surrounding coma should be essentially the same. On the other hand, the organized motions of dust particles are diagnostic of the place of origin and the column densities of dust are thus measures of its spatial density enhancement in the jet.

Application of an approximate deconvolution method, based on these considerations, 

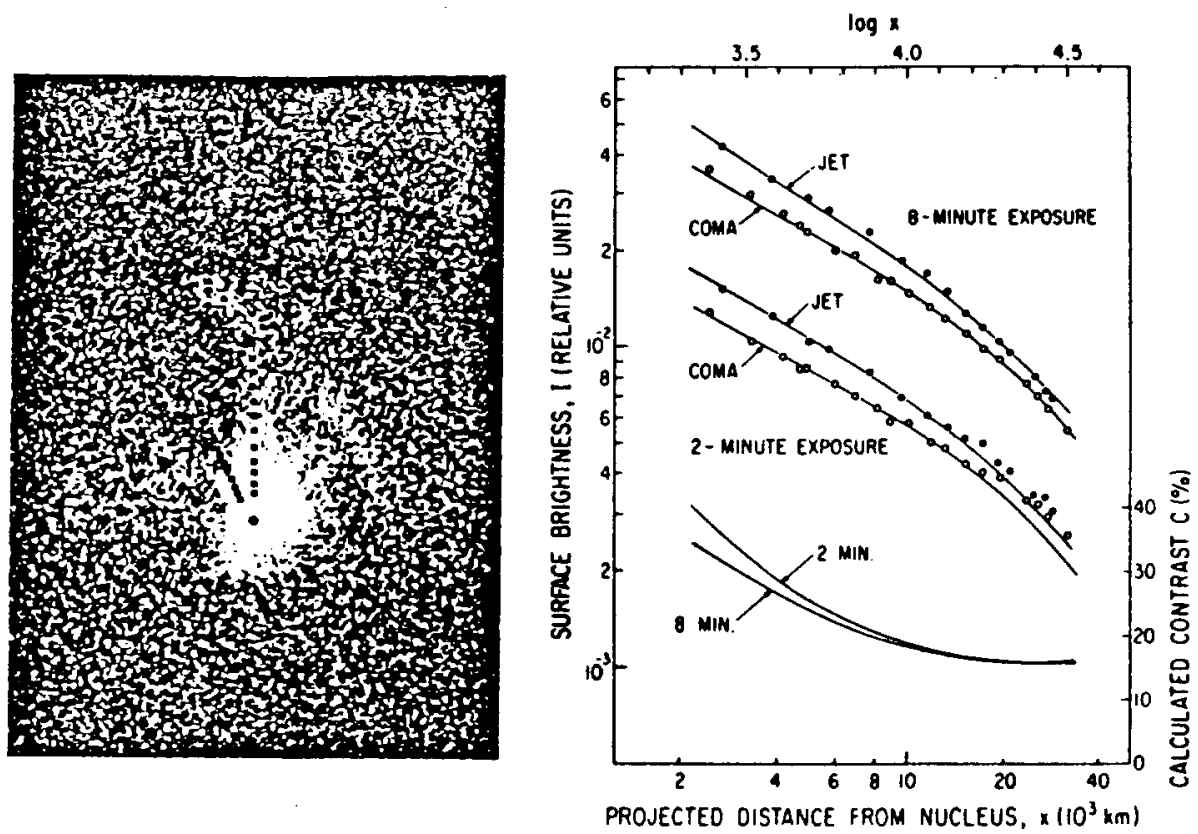

Fig. 28. Left: A digitized image of Comet Halley on one of the exposures taken on 1910 May 9 at Mount Wilson (Larson and Sekanina, 1984). The width of the frame is $60000 \mathrm{~km}$ and the direction to the Sun is up. The approximate positions at which the brightness was measured in the coma background and in the jet are indicated by the dark marks. Right, top: The profiles of the coma background and the jet on the two exposures (8-min and 2-min) of May 9. The measured relative surface brightnesses (left scale) of the coma background (open symbols) and of the jet (closed symbols) are plotted versus projected distance from the nucleus. Right, bottom: the jet's calculated contrast (right scale) relative to the coma background versus projected distance from the nucleus for the two exposures.

to the brightness profiles of the May 9 photographic images (Figure 28) has shown that $\mathrm{C}_{2}$ was likely to be a prevailing gaseous species and that the jet-to-coma contrast was $100 \%$ or more in the continuum, although the highest feature contrast measured directly was only about $30 \%$. Depending on the jet's sector angle (depth) along the line-of-sight, this result leads to particle number density in the jet from several to several tens of times the density in the surrounding coma at the same distance from the nucleus. Such high particle concentrations in jets could substantially reduce the contrast and visibility of the nucleus or portions thereof, as seen from spacecraft (cf. Section 9.4). The measured optical contrast also implies that a camera might confuse a jet with the nucleus, and that dust detectors on spacecraft may be exposed to highly erratic particle-impact rate variations with time (cf. Section 10.2).

\section{Radiation Model}

Electromagnetic radiation from a comet can be distinguished by source and character: typically the nucleus and dust are sources of reflected or scattered sunlight and of infrared thermal emission, whereas cometary gas molecules and ions are sources of 
discrete emission lines or bands extending from the UV through the visible, IR and radio ranges of the spectrum. The following sections relate this radiation to the source descriptions in prior chapters, and treat two particular considerations which have important radiation consequences for Halley investigations.

\subsection{RADIATION FROM COMETARY GAS}

Figures 9 and 10 illustrate the spectra of $P /$ Halley and a more recently observed, normal, moderately dusty, short-period comet, and Tables VI and VIII list transitions and species identified by their emissions in Halley and other comets (all in Section 6.1). Figure 10, in particular, is representative of the quantitative emission intensities which might be expected for Halley, if the appropriate adjustments for gas production and viewing geometry are made. Several atomic lines dominate the ultraviolet, the strongest being Lyman-alpha $(\mathrm{L} \alpha)$ of hydrogen at wavelength $121.6 \mathrm{~nm}$; this line and other emissions from atomic $\mathrm{H}$ are sufficiently unique to warrant separate treatment in Section 9.2. Other significant atomic emissions between $L \alpha$ and $200 \mathrm{~nm}$ come from oxygen, carbon, and sulfur. A few atomic emission lines also appear in the visible, the most significant by far being the forbidden lines of metastable oxygen at 630.0 and $636.4 \mathrm{~nm}$ (see caption to Table VIII). When Halley is near perihelion one should also expect to see very weakly the sodium D lines at 589.0 and $589.6 \mathrm{~nm}$; these have never been seen as far from the Sun as the encounter distance of the Halley probes. A number of metallic lines have been seen only in comets that approach extremely near the Sun (within $0.01 \mathrm{AU}$ ).

Flight project concern has been mainly with the light level in the coma that might be seen by imaging systems, especially by a CCD having sensitivity at wavelengths between 0.4 and $1.0 \mu \mathrm{m}$, and this range is emphasized in the gas emission considerations which follow (light levels throughout the spectrum from particulates are discussed in Section 9.3). In the visible the molecular bands (particularly including those from the dominant radicals; cf. Section 6.1 ) that have been studied in detail shine principally by resonance fluorescence. In this case, the luminance $I\left(W \mathrm{~m}^{-2} \mathrm{sr}^{-1}\right.$, equivalent to integral intensity in Section 9.3) for an entire emission line or band can be expressed in the form

$$
I=\frac{1}{4 \pi} \int_{0}^{\infty} \mathrm{d} s n g_{e} e^{-\left(\tau+\tau^{\prime}\right)} .
$$

Here the integration proceeds from the observer along the line-of-sight, where $n$ and $g_{e}$ are the local concentration $\left(\mathrm{m}^{-3}\right)$ and emission factor $(W)$ for the molecule and line or band in question, and $\tau$ and $\tau^{\prime}$ are the optical depths which separate the molecule from the observer and from the Sun, respectively. Values for $g_{e}$ are derived using both laboratory and spectrophotometric data by which the excitation path in the cometary coma can be understood, and Table XI lists values for $g_{e}$ and wavelength $\lambda$ for several important cometary emissions. As can be expected, $g_{e}$ is commonly proportional to $r^{-2}$, except that for some emissions the Doppler shift between the solar and absorption line 


\section{TABLE XI}

Values of emission factors for several molecules at $r=1 \mathrm{AU}$ (cf. also Tables VI and VIII). Most are nearly proportional to $r^{-2}$, and for some (as indicated in column 3) the value of $g_{e}$ is a strong function of the heliocentric velocity $\dot{r}=\mathrm{d} r / \mathrm{d} t$.

\begin{tabular}{|c|c|c|c|}
\hline $\begin{array}{l}\text { Molecule } \\
\text { (transition) }\end{array}$ & $\begin{array}{l}\text { Wavelength } \\
\lambda(\mathrm{nm})\end{array}$ & $\begin{array}{l}\text { Emission factor } \\
g_{e}(\mathrm{~W})\end{array}$ & Reference \\
\hline $\mathrm{OH}(0-0)$ & 306.4 & strong $f(\dot{r})$ & Schleicher and A'Hearn (1982) \\
\hline $\mathrm{OH}(1-1)$ & 312.2 & strong $f(\dot{r})$ & Schleicher and A'Hearn (1982) \\
\hline NH $(0-0)$ & 336.0 & strong $f(\dot{r})$ & Litvak and Kuiper (1982) \\
\hline $\mathrm{CN}(\Delta v=0)$ & 388.9 & strong $f(\dot{r})$ & Tatum and Gillespie (1977) \\
\hline $\mathrm{CN}(\Delta v=-1)$ & 421.6 & $3.4 \times 10^{-22}$ & $\begin{array}{l}\text { Tatum and Gillespie (1977) } \\
\text { and A'Hearn (1975) }\end{array}$ \\
\hline $\mathrm{C}_{3}$ (group) & 405.0 & $2.5 \times 10^{-21}$ & A'Hearn and Cowan (1975) \\
\hline $\mathrm{CH}(\Delta v=0)$ & 431.4 & $9.2 \times 10^{-21}$ & A'Hearn et al. (1980) \\
\hline$C_{2}(\Delta v=+2)$ & 438.2 & $1.4 \times 10^{-21}$ & A'Hearn $(1978,1982)$ \\
\hline $\mathrm{C}_{2}(\Delta v=+1)$ & 473.7 & $1.2 \times 10^{-20}$ & A'Hearn $(1978,1982)$ \\
\hline $\mathrm{C}_{2}(\Delta v=0)$ & 516.5 & $2.2 \times 10^{-20}$ & A'Hearn (1982) \\
\hline$C_{2}(\Delta v=-1)$ & 563.5 & $1.0 \times 10^{-20}$ & A'Hearn $(1978,1982)$ \\
\hline $\mathrm{C}_{2}(\Delta v=-2)$ & 619.1 & $3.2 \times 10^{-21}$ & A'Hearn $(1978,1982)$ \\
\hline$O\left[{ }^{1} D-{ }^{3} P\right]$ & 630.0 & $1.7 \times 10^{-21}$ & Spinrad (1982) \\
\hline $\mathrm{NH}_{2}$ (many) & many & for 6 bands & A'Hearn (1982) \\
\hline
\end{tabular}

profiles for the exciting photons introduces a strong dependence on heliocentric velocity (as noted in Table XI; the Swings effect is discussed by A'Hearn, 1982).

Within the inner few thousand $\mathrm{km}$ of the coma the detailed dynamics and thermal structure of the dust and gas require simultaneous inputs from chemistry, thermodynamics, radiation transfer theory, and hydrodynamics (e.g., Marconi and Mendis, 1983, and Sections 5 and 6 of this review). Many of the boundary conditions and parameters in the controlling mathematical equations are poorly known or even unknown. Because of these difficulties the models used to interpret observations generally have been simplified, even oversimplified. The best existing models for interpreting the gas emissions are those of Festou (1981a, b), of Combi and Delsemme (1980), and of Keller (1976). At visible wavelengths the older Haser (1957) formulation can be used for general calculations of light levels. As based on Equation (6.8), this approach has the advantages of simplicity and ease of computation (Section 6.3).

Isophotes of bright comets such as Halley are sensibly symmetric near the nucleus as seen in the light of their radicals (e.g., Rahe et al., 1976; Hognar and Richter, 1980). Two factors can contribute to the presence of significant gas emission from the dark side of the nucleus. One is heating of the dark side by infrared radiation from the dust coma (Weissman and Kieffer, 1981), and the other is simple migration to the dark side as the result of molecular collisions. The fact that dust jets appear to originate only on the sunward side of Halley (Sekanina and Larson, 1984) makes the latter explanation more likely to dominate. Thus, for most cometary observations of molecular emissions the assumptions that their concentration $n$ is spherically symmetric, that $g_{e}$ is independent of path length $s$, and that the optical depths ( $\tau$ and $\tau^{\prime}$ in Equation (9.1)) 
are negligible, are all quite good, and Equation (9.1) becomes (Chamberlain, 1961, Chapter 11)

$$
I=\frac{g_{e}}{4 \pi} N
$$

Here the column density $N\left(\mathrm{~m}^{-2}\right)$ of emitting molecules along the line-of-sight depends only on the cometocentric distance of the observer and on the impact parameter $x$ of the line-of-sight; the appropriate expressions for $N$ are given by Haser (1957) and by Newburn (1983; Equations (11) and (12)) for the inward- and outward-looking cases. When the observer is outside the emitting region, Newburn's (1983) expression for the inward-looking value of $N$ becomes only a function of $x$, and of the appropriate scale lengths (cf. Equation (6.8) and Table VIII), molecular outflow velocity $u\left(\mathrm{~m} \mathrm{~s}^{-1}\right)$, and production rate $Q_{p}\left(\mathrm{~s}^{-1}\right)$, in the form

$$
N=\frac{Q_{p}}{2 \pi x u_{d}} \frac{l_{d}}{l_{d}-l_{p}} \int_{x / l_{d}}^{x / l_{p}} K_{0} \mathrm{~d} y,
$$

where the argument of $K_{0}$ (modified Bessel function of the second kind) is the dimensionless integration variable $y$.

For a parent molecule, Equation (9.3) becomes (Haser, 1957)

$$
N=\frac{Q_{p}}{2 \pi x u_{p}}\left[\frac{\pi}{2}-\int_{0}^{x / l_{p}} K_{0} \mathrm{~d} y\right] .
$$

One can derive a column density $N$ for water molecules from Equation (9.4) using a scale length $l_{p}$ near $8 \times 10^{4} \mathrm{~km}$ that will produce a useful approximation for this dominant parent molecule (Festou, 1981b). It is dominant in number only, and not in flux. The water molecule has been directly observed (for one comet) only at radio wavelengths, although its ion $\mathrm{H}_{2} \mathrm{O}^{+}$is relatively prominent in the red part of the spectrum of the outer coma and ion tails.

Parent molecules other than water are not well known, although they very probably include $\mathrm{CO}_{2}, \mathrm{CS}_{2}, \mathrm{NH}_{3}, \mathrm{HCN}, \mathrm{CH}_{3} \mathrm{CN}$, and possibly $\mathrm{CO}$ (Feldman, 1983; Altenhoff et al., 1983). They are largely destroyed near the nucleus as compared to the radicals, whatever their production rates. Ultraviolet lines and bands resulting from these and other parents are fairly well understood and have been modeled (Feldman, 1982). Considerable modeling has also been done of the fundamental and low overtone vibration bands of possible parent molecules at infrared wavelengths (Yamamoto, 1982; Encrenaz et al., 1982; Crovisier and Encrenaz, 1983; and Weaver and Mumma, 1984). In general these bands are virtually lost in the strong dust continuum and can only be detected with a high resolution spectrometer. They contribute insignificantly to the overall flux level. 
When the concentration of radicals in the coma cannot be described by a simple mathematical expression (or that expression cannot be analytically integrated to obtain the column density), then the column density must be obtained by numerical integration along the line of sight, assuming that some concentration map exists. Haser-type expressions for $N$ for a nucleus with variable production across the surface are given by Newburn (1983).

\subsection{RESONANCE RADIATION OF HYDROGEN}

The strongest resonance line of atomic hydrogen is $L \alpha$ at wavelength $\lambda=121.6 \mathrm{~nm}$. The exciting solar line is strong, broad, and self-reversed. The two peaks can be approximated by overlapping Gaussians. The differential flux at the solar line center will be about $3.5 \times 10^{11}$ photon $\mathrm{cm}^{-1} \mathrm{~s}^{-1} \mathrm{~A}^{0-1}$ near the solar cycle minimum when Halley reaches perihelion. The flux at the peaks of the solar line is about $50 \%$ larger. The line is broad enough to excite all cometary hydrogen independent of the radial velocity of the comet.

Earlier observations of $\mathrm{L} \alpha$ emission from extended hydrogen comae (Bennett, 1970 II, Kohoutek, 1973 XII, West, 1976 VI) have been analysed using detailed models developed by Keller and Thomas (1975) and Keller and Meier (1976, 1980), and reviewed by Keller (1976). A similar model has been applied to potential observations of Halley for several dates in 1985 and 1986 by Meier and Keller (1985). In this model it was assumed that Halley's total gas production consists only of water molecules

\section{LOG INTENSITY (R)}

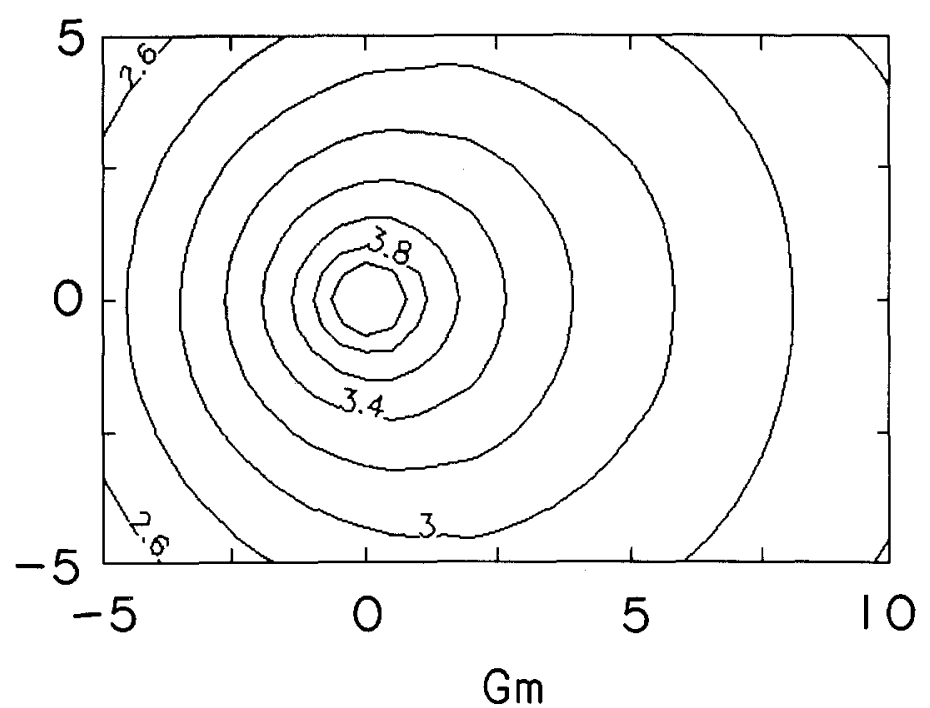

Fig. 29. Isophotes of Halley hydrogen $\mathrm{L} \alpha$ emission as predicted for observation from Earth on 1986 March 13. The projected coordinates (in $\mathrm{Gm}=0.0067 \mathrm{AU}$ ) have the nucleus at the origin and the Sun to the left. Contours are identified by the (base ten) logarithm of the intensity (1 Rayleigh corresponds to an intensity of $25 / \pi$ photons $\mathrm{m}^{-2} \mathrm{~s}^{-1} \mathrm{sr}^{-1}$ ) for the sum of the 8 and $20 \mathrm{~km} \mathrm{~s}^{-1}$ components. 
whose dissociation divides the hydrogen atoms equally into two Maxwellian velocity distributions centered on radial outflow speed of 8 and $20 \mathrm{~km} \mathrm{~s}^{-1}$ (cf. Section 6.3); the total production is $Q_{\mathrm{H}}=1.2 \times 10^{30} \mathrm{~s}^{-1}$ on 1986 March 13. Sample results include Figure 29 which shows the expected $L \alpha$ intensity distribution for the hydrogen coma as seen from Earth on this date. Along the Sun-comet line the extent of the coma is about $15 \mathrm{Gm}$ at intensity levels which exceed that of the interstellar hydrogen background. The influence of radiation pressure (which forces the atoms away from the Sun, just as for the dust tail; cf. Section 6.3 and 7.4) is clearly visible in the asymmetry of the isophotes. Depending on the solar activity (through the strength of the solar $L \alpha$ line), the radiation pressure force can exceed the solar gravitational attraction. Curvature of the isophotes, as manifested by an asymmetry relative to the Sun-comet line, is barely visible.

For planning and interpretation of Halley observations, Figure 30 predicts isotachs for the radial velocity components relative to Sun (for Swings and Greenstein effects) and to Earth (for assessment of geocoronal absorption), isochrones for the mean lifetimes of the $\mathbf{H}$ atoms, and isophotes for the intensities of both the 8 and $20 \mathrm{~km} \mathrm{~s}^{-1}$ components. Averaging along the line of sight has been included in these results. For 1986 March 13 the radial velocity components relative to Earth are far enough from zero that there is no absorption by geocoronal $\mathrm{H}$ atoms. However, emission from geocoronal atoms must be taken into account for Earth orbiting spacecraft, depending on the observational geometry.

The cometary $\mathrm{L} \alpha$ emission line has a typical full width at half maximum (FWHM) near $0.006 \mathrm{~nm}$ (Drake et al., 1976), about 0.05 times that of the solar line. The low velocity atoms dominate because of their higher density (cf. Equation (6.8)). The optical thickness of the $L \alpha$ radiation exceeds unity in the inner parts (within $1 \mathrm{Gm}$ ) where the $\mathrm{L} \alpha$ intensity exceeds about $30 \mathrm{kR}$ (at $1 \mathrm{AU}$ ). In this region the intensity increases slower than $x^{-1}$ (impact parameter $x$, cf. Equation 9.3). Multiple scattering becomes important in this inner region and the data interpretation is accordingly more complex (Keller, 1973).

The solar $\mathrm{L} \beta$ emission at wavelength $102.5 \mathrm{~nm}$ yields scattered $\mathrm{L} \beta$ photons. About $12 \%$ of the $\mathrm{L} \beta$ quanta are absorbed and the energy is divided between $\mathrm{L} \alpha$ and Balmer $(\mathrm{H} \alpha$ at $656.2 \mathrm{~nm})$ quanta. The intensity of the optically thin part of the coma in $\mathrm{L} \beta$ can be scaled by multiplying the $\mathrm{L} \alpha$ intensity by a factor $2 \times 10^{-3}$, and the comparable factor for $\mathrm{H} \alpha$ is $2.7 \times 10^{-4}$. If different hydrogen production rates are considered (there are indications that the nominal model predicts values which are too small), the isophote intensity values (Figures 29 and 30, upper panels) must be multiplied by the appropriate ratio.

\subsection{RADIATION FROM THE DUST}

To discuss radiative transfer by the dust particles we outline a continuum description assuming unpolarized radiation for which the variations with wavelengths occur only on scales for which $\mathrm{d} i / \mathrm{d} \lambda$ does not much exceed $i / \lambda$. Here the independent variables are time, position, direction and wavelength $\lambda$ of observation, and the major dependent variables are differential intensity $i$ (units $\mathrm{W} \mathrm{m} \mathrm{m}^{-2} \mu \mathrm{m}^{-1} \mathrm{sr}^{-1}$ ) and flux $j$ 
13 MAR 86
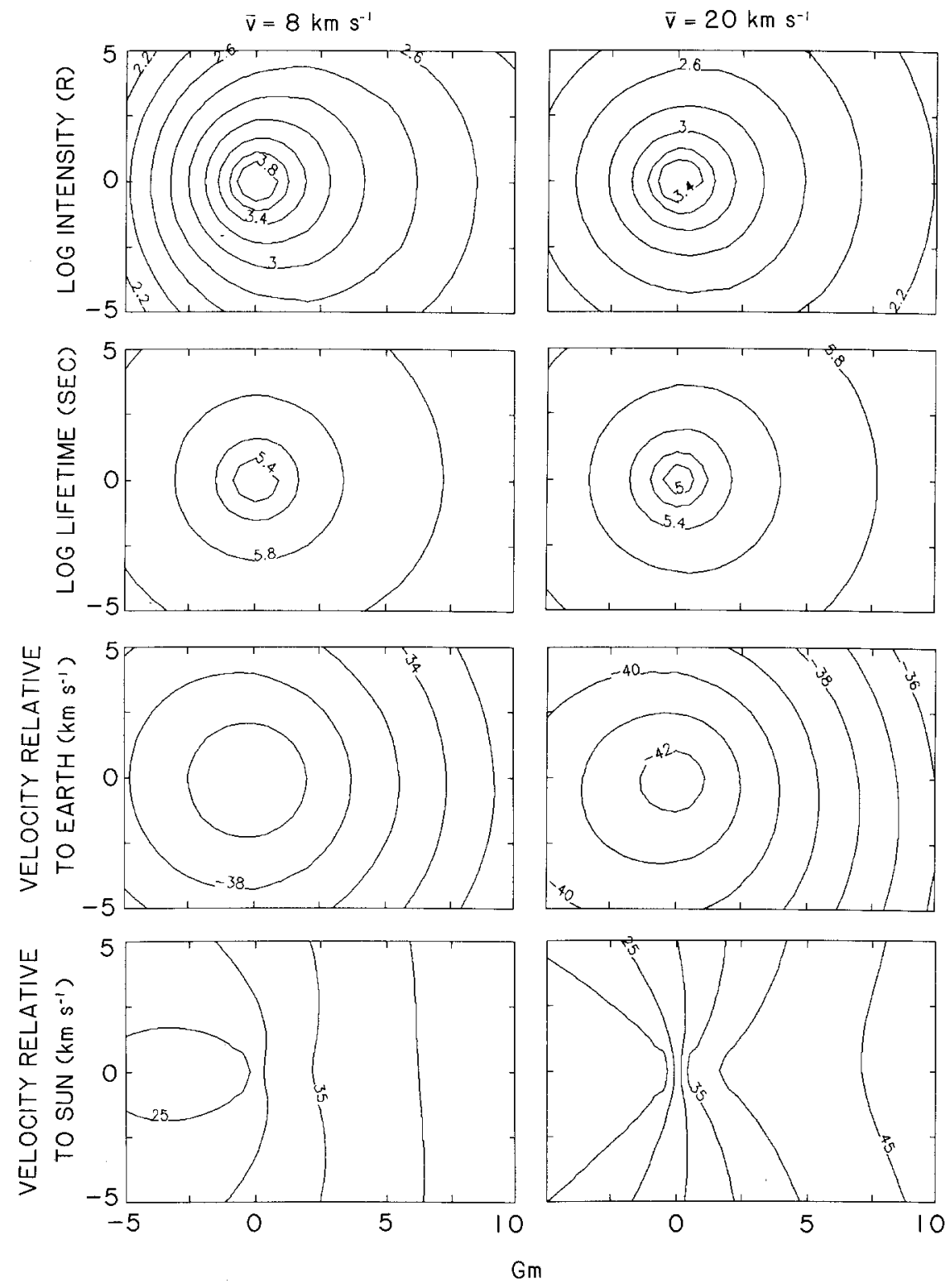

Fig. 30. Model predictions for $\mathrm{L} \alpha$ isophotes, isochrones and isotachs. Coordinates and observing conditions are described in the caption to Figure 29, and the 8 and $20 \mathrm{~km} \mathrm{~s}^{-1}$ components are shown separately in the left and right panels, respectively.

( $\mathrm{W} \mathrm{m}^{-2} \mu \mathrm{m}^{-1}$ ). To distinguish the special roles played by wavelength $\lambda$ and dust particle radius $a$ from those of other quantities having the dimension of length, we cite their units as ' $\mu \mathrm{m}$ ' and 'size', respectively, in this analysis. 
To start, consider an isolated dust particle in sunlight, described by a constant unidirectional differential flux ( $\left.\mathrm{W} \mathrm{m}^{-2} \mu \mathrm{m}^{-1}\right)$

$$
j_{\odot}=\frac{L_{\odot}}{4 \pi r^{2}} f_{\odot} e^{-\tau^{\prime}}
$$

Here $r$ and $\tau^{\prime}$ are the distance and optical depth which separate the particle from the Sun, and $f_{\odot}$ represents the wavelength dependence of sunlight without extinction. An excellent approximation to $f_{\odot}$ is provided by a Planck function at temperature $T_{\odot}=5770 \mathrm{~K}$, and the normalization

$$
\int_{0}^{\infty} \mathrm{d} \lambda f_{\odot}=1,
$$

allows us to represent the luminosity of the Sun using $L_{\odot}=3.83 \times 10^{26} \mathrm{~W}$. For the dust particle the extinction cross section $\sigma_{x}\left(\mathrm{~m}^{2}\right)$, single scattering albedo $A$ (cf. Section 7.1), and emissivity $\varepsilon$ are defined so that expressions for the power per unit wavelength ( $\mathrm{W} \mu \mathrm{m}^{-1}$ ), respectively, removed from the beam, scattered and emitted by the isolated particle in sunlight become

and

$$
\begin{aligned}
& l_{x}=\sigma_{x} j_{\odot}, \\
& l_{s}=A \sigma_{x} j_{\odot},
\end{aligned}
$$

$$
l_{e}=\left(4 \varepsilon \sigma_{x} / Q_{\text {ext }}\right) \sigma T^{4} f .
$$

In Equation (9.9), the factor 4 follows from the approximation of the dust grains by solid spheres, and $f$ is normalized as is $f_{\odot}$ (Equation (9.6)) but applies to the local grain temperature $T(\mathrm{~K})$. For each isolated particle this temperature is determined by the radiative energy balance in the form

$$
\int_{0}^{\infty} \mathrm{d} \lambda\left(l_{x}-l_{s}\right)=\int_{0}^{\infty} \mathrm{d} \lambda l_{e} .
$$

For absorbing grains this balance leads to dust temperatures of the order of $300 \mathrm{~K}$ at a distance $r=1 \mathrm{AU}$ from the Sun, whereas dielectric grains can be at least $100 \mathrm{~K}$ colder. Thus the thermal radiation $l_{e}$ (Equation (9.9)) has its peak at wavelengths near $10 \mu \mathrm{m}$, well separated from that of $j_{\odot}, l_{x}$, and $l_{s}$ (near $0.6 \mu \mathrm{m}$; cf. Equations (9.5) through (9.8)). We can thus deal approximately independently with the visible and infrared spectra of the dust in scattered and emitted radiation, respectively.

An observer separated from the particle by a large distance $s$ (where $s^{2} \gg \sigma_{x}$ ) receives the differential flux $\left(\mathrm{W} \mathrm{m}^{-2} \mu \mathrm{m}^{-1}\right.$ )

$$
j=\frac{4 \pi l_{s} \Phi_{s}+l_{e}}{4 \pi s^{2}}=\frac{\sigma_{x}}{s^{2}}\left[A \Phi_{s} j_{\odot}+\frac{\varepsilon \sigma T^{4} f}{\pi Q_{\mathrm{ext}}}\right] .
$$


Here the scattering function $\Phi_{s}\left(\mathrm{sr}^{-1}\right)$ describes the dependence of the scattered flux on scattering angle $\theta_{s}$ and the emission is assumed isotropic. We require conservation of energy for each wavelength in the outgoing radiation in the form

$$
\int_{4 \pi} \mathrm{d} \Omega s^{2} j=l_{s}+l_{e},
$$

such that the coefficients used in Equation (9.11) reflect a normalization for $\Phi_{s}$ in the form

$$
\int_{4 \pi} \mathrm{d} \Omega \Phi_{s}=2 \pi \int_{0}^{\pi} \mathrm{d} \theta_{s}\left(\sin \theta_{s}\right) \Phi_{s}=1
$$

Observations of cometary dust require that the scattering function $\Phi_{s}$ have the properties discussed in Section 7.1.

We next consider a distribution of dust grains, and use the power removed from the beam (Equation (9.7)) to obtain the expression

$$
\tau=\int_{S_{1}}^{S_{2}} \mathrm{~d} s \sum_{k} \int_{0}^{\infty} \mathrm{d} a n \sigma_{x}
$$

for the optical depth separating two positions at $s_{1}$ and $s_{2}$ along a line-of-sight. We use $\tau$ to represent the optical depth from the dust to the observer, $\tau^{\prime}$ dust to Sun (as in Equation (9.5)), $\tau_{N}$ nucleus to observer, and $\tau_{N}^{\prime}$ nucleus to Sun. In Equation (9.14) the sum and the second integral allow for various kinds $k$ of particles, each distributed in size $a$, with local differential concentration $n\left(\mathrm{~m}^{-3} \operatorname{size}^{-1}\right.$; cf. Equation (7.13) in Section 7.4) as a function of pathlength $s(\mathrm{~m})$ along the line-of-sight. The product $s^{2} n$ thus represents the number of dust grains per unit size, solid angle, and pathlength as seen by an observer, and the differential intensity $i\left(\mathrm{~W} \mathrm{~m}^{-2} \mu \mathrm{m}^{-1} \mathrm{sr}^{-1}\right)$ is related to the flux $j$ in Equation (9.11) by

$$
i=\int_{0}^{\infty} \mathrm{d} s s^{2} e^{-\tau} \sum_{k} \int_{0}^{\infty} \mathrm{d} a n j .
$$

Here the limits in the pathlength integration for $\tau$ (Equation (9.14)) become 0 and $s$. This expression (Equation (9.15)) would suffice for the observed intensity were it not for scattering by each particle of radiation from its neighbors and from other sources (e.g., the cometary nucleus). To include these effects we replace Equation (9.15) by

$$
i=\int_{0}^{\infty} \mathrm{d} s e^{-\tau} \sum_{k} \int_{0}^{\infty} \mathrm{d} a n \sigma_{x}\left[A \Phi_{s} j_{\odot}+\frac{\varepsilon \sigma T^{4} f}{\pi Q_{\text {ext }}}+A \int_{4 \pi} \mathrm{d} \Omega \Phi_{s} i\right] .
$$

The presence of the local intensity $i$ at each end of Equation (9.16) makes a major difference in the complexity of its solutions depending on whether the last, multiple 
scattering term inside the integrals can be neglected. If not, as is the case for dust optical depths of the order unity or larger, numerical iterative solutions are essential. Hellmich and Keller (1981b), Weissman and Kieffer (1981), and others have each performed self-consistent calculations including this term, and use the results to investigate the way in which the radiation feedback mechanism influences the nucleus and dust temperatures. As discussed at the beginning of Section 9.4, large optical depths occur only within a few $\mathrm{km}$ of the nucleus' surface, so that multiple scattering can often be neglected for the interpretation of remote sensing observations in which the nuclear region subtends a small fraction of the field of view.

For isotropic radial dust outflow at constant production $Q$ and effective velocity $V$, Equation (9.14) implies that the optical depth $\tau$ be proportional to $Q / V x$ for an external observer whose line of sight has impact parameter $x$ from the center of the nucleus (of course these assumptions are poor for small $x$, of the order of a few nuclear radii, but they often have a reasonably large range of validity). If we could also neglect both $\tau^{\prime}$ and multiple scattering, and assume that $T, \varepsilon, A, Q_{\text {ext }}, \Phi_{s}$, and $j_{\odot}$ were all independent of dust particle size and of position within the coma, Equation (9.16) would take the simple form

$$
i=\left[A \Phi_{s} \frac{L_{\odot} f_{\odot}}{4 \pi r^{2}}+\frac{\varepsilon \sigma T^{4} f}{\pi Q_{\mathrm{ext}}}\right]\left(1-e^{-\tau}\right)
$$

For small values of optical depth $\tau$, this expression is also proportional to $Q / V x$ (just as for the gas emission in Equation (9.2) through 9.4).

Other radiation quatities of interest can be derived from the intensity $i$ by integration. Among these are the differential flux $\left(\mathrm{W} \mathrm{m}^{-2} \mu \mathrm{m}^{-1}\right)$

$$
j=\int_{4 \pi} \mathrm{d} \Omega W_{\alpha} i
$$

integral intensity $\left(\mathrm{W} \mathrm{m}^{-2} \mathrm{sr}^{-1}\right)$

$$
I=\int_{0}^{\infty} \mathrm{d} \lambda W_{\lambda} i
$$

and integral flux $\left(\mathrm{W} \mathrm{m}^{-2}\right)$

$$
J=\int_{0}^{\infty} \mathrm{d} \lambda \int_{4 \pi} \mathrm{d} \Omega W_{\alpha} W_{\lambda} i
$$

Here the dimensionless weighting functions $W_{\alpha}$ and $W_{\lambda}$ depend on direction and on wavelength, respectively; for example, the use of $W_{\alpha}=1$ for all angles in Equations (9.18) and (9.20) would yield omnidirectional fluxes $j$ and $J$. Using the product $W_{\alpha} W_{\lambda}$ of the directional and spectral sensitivities of a detector enables the corresponding value of the integral flux $J$ (Equation (9.20)) to be proportional to the detector 
response. In this case, using $m_{s}$ to represent the astronomical magnitude of a standard point source (star), having its differential flux $j_{s}$ when centered in the detector field of view (where $W_{\alpha}=1$ ), we would obtain

$$
m=m_{s}+2.5 \log \left[\frac{1}{J} \int_{0}^{\infty} \mathrm{d} \lambda W_{\lambda} j_{s}\right],
$$

for the magnitude of an observed distribution of cometary dust. The difference between magnitudes which use different spectral sensitivity functions $W_{\lambda}$ thus becomes a measure of the color of the dust radiation. Equations (9.20) and (9.21) demonstrate that significant variations of cometry intensity $i$ with direction (i.e., with projected position in the coma, as included in a detector aperture function $W_{\alpha}$ ) and with wavelength (e.g., as gaseous emission lines are or are not included in the response function $W_{\lambda}$ ) may be responsible for the unique requirements which apply to quantitative measurements and interpretations of cometary remote sensing (as noted in several chapters of this review). Additional considerations, including limitations introduced by atmospheric extinction, seeing, telescopic resolution, photographic non-linearity, and others, further compromise the results; Section 9.5 provides an interesting example.

Sample values using equations similar to the above for Halley near $1 \mathrm{AU}$ are given by Divine (1981b) and Moroz (1982). Even though these analyses yield quite diverse values for some quantities (e.g., optical depth to the nucleus' surface), the range of dust intensity seen by an external observer looking at the center of the coma (at $x=0$ ) is modest, of the order of 4 and $0.3 \mathrm{~W} \mathrm{~m}^{-2} \mu \mathrm{m}^{-1} \mathrm{sr}^{-1}$ for such models at wavelengths 0.6 and $15 \mu \mathrm{m}$ in the visible and infrared. For an observer at $s=1 \mathrm{AU}$, magnitude +4 (cf. Figure 3) corresponds to a differential flux $8 \times 10^{-10} \mathrm{~W} \mathrm{~m}^{-2} \mu \mathrm{m}^{-1}$ at $\lambda=0.6 \mu \mathrm{m}$ if the dust is the major contributor. Figure 19 illustrates the profile of mean instensity from one Halley model, for observers within the coma. Attempts are presently in progress to modify the dust and radiation models to make them eventually consistent with the observations.

\subsection{VisIBILITY OF THE NUCLEUS}

The visibility of the nucleus is impaired primarily by the dust, as the gas contributes only in selective resonance bands (Section 9.1). The interference of the dust grains with the radiation reaching and leaving the nucleus depends both on their optical properties (Section 7.1) and, more strongly, on their geometrical distribution, i.e., the concentration of the grains. Very close to the nucleus (up to 10 radii) their distribution is determined by the drag forces of the sublimating gas molecules lifting grains from the surface and accelerating them to their terminal velocities (cf. Section 5). Therefore, their concentration peaks very strongly just above the surface and most of their contributions to the opacity and competing intensity will occur in this hydrodynamic interaction region.

Consider a nucleus separated from the Sun and from the observer by the distances $r$ and $s$ and by the optical depths $\tau_{N}^{\prime}$ and $\tau_{N}$ (cf. Equation (9.14)). We use the Bond 
albedo $A_{N}$ for the nucleus (Hanner et al., 1981, provide a careful discussion of the relation between Bond and single scattering albedo), and assume that the nucleus' intensity is isotropic and uniform over the sunlit hemisphere, as given by

$$
i_{N}=\frac{A_{N}}{2 \pi} \frac{L_{\odot} f_{\odot}}{4 \pi r^{2}} \exp \left(-\tau_{N}^{\prime}-\tau_{N}\right) .
$$

The ratio of the visible contributions in Equations (9.22) and (9.17), namely

$$
\frac{i_{N}}{i}=\frac{A_{N}}{2 \pi A \Phi_{s}} \frac{e^{-\tau_{N}^{\prime}}}{e^{\tau_{N}}-1},
$$

is a measure of the nucleus' visibility. The quantity

$$
A_{E}=\frac{A_{N}}{2 \pi A \Phi_{s}},
$$

can be interpreted as an effective albedo of the nucleus in the presence of dust, and is a useful model parameter.

The approach represented by Equations (9.23) and (9.24) has been used by Hellmich and Keller (1981a) for a geometry in which the observer is directly between the nucleus and the Sun (thus $\theta_{s}=\pi$ and $\tau_{N}^{\prime}=\tau_{N}$ ). Their investigation was based on a size distribution of olivine grains suggested by Sekanina and Miller (1973). Sample calculations for a total dust production rate $Q_{d}=4.6 \times 10^{6} \mathrm{~g} \mathrm{~s}^{-1}$ from a nucleus with radius $R_{N}=2.5 \mathrm{~km}$ and Bond albedo $A_{N}=0.3$ showed that $\tau_{N} \simeq 0.59$ and $i_{N}<i$ in the visible. The presently adopted model of Halley at the time of the spacecraft encounters assumes the rather similar values $R_{N}=3 \mathrm{~km}$ and $Q_{d}=4.7 \times 10^{6} \mathrm{~g} \mathrm{~s}^{-1}$. However, our perception of the nature of the grains and their size distribution has changed appreciably. The single scattering albedo of the grains is now $A=0.05$ (compared to $A \simeq 1$ for olivine, as used in the above calculation), and the cut-off for small particles is now $a=0.1 \mu \mathrm{m}$ rather than $0.46 \mu \mathrm{m}$ for the Sekanina and Miller distribution. Neglecting the differences and assuming that the optical thickness along the line of sight remains at $\tau_{N}=0.59$, the old results can be scaled to the now assumed lower albedos. Using the present values for the nucleus $\left(A_{N}=0.12\right)$ and for the dust $\left(A=0.05, \Phi_{s}=0.069 \mathrm{sr}^{-1}\right)$, the effective albedo $A_{E}$ (Equation (9.24)) changes from 0.6 to 5.5. This yields then by scaling $i=(0.26) i_{N}$ at $\lambda=600 \mathrm{~nm}$ rather than the old value of $(2.4) i_{N}$.

This rough scaling of the earlier results indicates that the predicted visibility of the nucleus has improved if the most recent parameter values for the Halley model are valid. However, it is clear that the actual degree of obscuration of the nucleus will depend on the details of the dust grain distribution in size and in location as well as on the scattering properties of the grains and the nucleus. The sensitivity of Equations (9.23) and (9.24) to small changes in parameter values may sway the visibility from fair to bad. 


\subsection{CENTER-OF-LIGHT/CENTER-OF-MASS OFFSET}

A classic problem of cometary astrometry is deciding where to place the measuring engine crosshairs on the cometary image. Photographic plates of bright comets have often been overexposed, either inadvertently, or deliberately to bring out the tails. The tendency in such cases is to attempt to place the crosshairs at the geometric center of the blob constituting the inner coma. When the comet has an asymmetric coma, the geometric center is far from the point of greatest intensity, the point from which the coma is presumably emitted and which presumably contains the nucleus. Roemer (1963) understood this hoary problem very well and reviewed it accordingly. Thus experienced astrometric observers either use a modern electronic detector with large dynamic range and 'set' on the brightest point, or take photographs exposed just long enough to begin to bring up the image. The real center-of-light/center-of-mass problem is more subtle and has two parts. So-called secondary nuclei and jets are temporary phenomena, apparently understood to some extent. The other part is the possible presence of real offsets of the center-of-mass from the center-of-light (understood as having the greatest intensity) seen in some or all wavelengths.

For Halley, Bobrovnikoff (1931) calls attention to numerous secondary nuclei and brilliant jets, the implication being that they were as bright or brighter than the real nucleus, although no real photometry exists. Sekanina and Larson (1984) have shown that both secondary nuclei and jets are often part of the same phenomenon viewed at different stages of evolution. Their source is a region of abnormal activity on the nucleus which throws out a column or fan of gas with extraordinarily high mass loading of dust. Their shape and motion are entirely explained by dust in the solar radiation field. They do not persist when the source region is out of sunlight (at night) but may recur on subsequent rotations from the same source.

Secondary nuclei and jets are usually no problem for ground-based astrometry specialists. All are readily recognizable after they have developed for a day or so, both from their appearance and from their nongravitational motion. When a jet is just beginning, however, and is not yet distinguishable as such from the ground, especially in less than perfect seeing, it could cause a bias. In 1862 Bond noted in his observing notebook on $P /$ Swift-Tuttle that jets from the comet could readily be confused as part of the nucleus. From the ground such positions (especially when observed with a small instrument) can be often discarded because they do not agree with the majority of the other data.

A ball of one meter radius dispersed into spheres of radius $0.2 \mu \mathrm{m}$ contains over $10^{20}$ particles and a (monolayer) cross section near $16 \mathrm{~km}^{2}$ ! Thus if occasional large particles are lifted from Halley's nucleus and come apart in sunlight as their ice glue evaporates, or if heavily mass loaded dust columns are common (and they appear to be), then some very bright features will appear in Halley's coma. Such features may propagate outward at a few hundred $\mathrm{m} / \mathrm{s}$ and could easily confuse a target-body-tracker.

Unfortunately we have no real knowledge of the structure of jets, nor of their surface brightness near the nucleus. Far from the nucleus, as they disperse and become optically thin, the jets should pose no problem. If this dispersion occurs close enough to the 
nucleus for the latter to remain in the field of viewing instruments, any prior confusion becomes immaterial. The best target-body-tracker may be one which studies either the geometric center or photometric center and the brightest single point, following the latter if it doesn't deviate too far from the former.

More than three decades ago Whipple (e.g., Hamid and Whipple, 1953) was already aware of potential trouble from a second part of the problem, the possible presence of real offsets between the center-of-light and the center-of-mass. There is an extensive collision zone in the inner coma in which gases interact with gases and with dust, but in which dust rarely interacts with dust (Section 5). The dust then flows radially outward on the sunward side until gradually deviated by radiation pressure (Section 7.4). Ignoring large fragments which 'come unglued' and create secondary nuclei (as reviewed by Sekanina, 1982), the dust flow then is strictly divergent, and the point of greatest brightness associated with it is at its source, the nucleus. The dust does appear to evolve dominantly from the sunward hemisphere, so viewed with a system that cannot resolve the nucleus, there would be a very small sunward bias in the apparent center just due to the poor resolution, unless viewed at zero phase angle. This would hold true even for a bare, inactive nucleus if asymmetrically lighted. The offset should be very small, certainly less than the radius of the nucleus.

The parent molecules sublimating from the nucleus are destroyed by several processes, the dominant one generally being photodissociation. Their concentration at any given distance from the nucleus is a product of their rate of divergence and one or more loss exponentials (as in Equation (6.7)). The one caveat that needs to be acknowledged is that there could be sources (e.g., dust, dust with ice inclusions, or ice grains) which release gas molecules over an extended volume of the coma. There is evidence that such grains exist in some comets far from the Sun (Hanner, 1984a). Near the Sun (at Halley/spacecraft encounter distances) they could exist out to a few hundred $\mathrm{km}$ at most (Hanner, 1981). Because no parent molecule emits light significantly at visible wavelengths, such extended sources would be important only if they produce daughter radicals that could be observed.

The parent molecules themselves probably are released predominantly on the sunward side of the nucleus, although heating of the dark side by coma dust grains may allow some release there as well (Weissman and Kieffer, 1981). Anti-sunward of the nucleus there are probably few dust grains to do the heating, even considering grains at large distances that have been turned back by radiation pressure. Collisions in the inner coma will cause a large migration of gas into the rear hemisphere. In the absence of detailed studies one can surmise that there might remain some preference of the molecules for the sunward hemisphere, especially if there were an extended grain source for them.

The gaseous radicals will begin with any distributional bias shown by their parents. Their continuing creation serves to flatten their $R^{-2}$ divergence near the nucleus, but even number densities peak near the nucleus, and the column density $N$ (proportional to the intensity, Equation (9.2)) shows no peaks (although it can be quite flat as a function of $x$ in the inner coma). 
Light from the comet is the simple sum of all the individual contributions, assuming that the optical depth remains small. The innermost few radii of the coma may in fact contain optically thick dust (Hellmich and Keller, 1981a, b). For the resolution possible with Earth-based telescopes, the coma at wavelengths 0.35 to $1.0 \mu \mathrm{m}$ is optically thin above a point-like source which probably includes some dust as well as the nucleus. At 0.9 AU heliocentric distance far more light will come from gas than from the dust (cf. Equation (3.3)). By selecting a wavelength at which there are no emission bands, this light can be removed. The remaining point of greatest intensity will be the nucleus plus at most a few nuclear radii of dust biased toward the Sun. It seems most unlikely that this bias could exceed $20 \mathrm{~km}$, about 0.02 arc sec as seen from Earth in early March 1986. In contrast, the geometric center of a large dust isophote might well be biased hundreds or thousands of kilometers sunward of the nucleus.

Delsemme and Combi (1983) have studied the profiles of dust and gas in Comet Kohoutek as a function of distance from the nucleus and have found that the dust profiles are biased sunward relative to the gas profiles. This result may well be the product of the more anisotropic emission of dust uncompensated by collisional migration to the antisunward direction, essentially as they suggest. The result does not obviously support their conclusion that the nucleus is to be found at the geometric center of the gas profiles, however, which may themselves be significantly biased by several physical processes. Their model leads to a dust apex distance of only $3600 \mathrm{~km}$ at heliocentric distance $r=0.465 \mathrm{AU}$. The equivalent apex distances for heliocentric distances at which much Halley data exist would then be only 7200 to $20000 \mathrm{~km}$, while observed dust structures at these times extend to at least $50000 \mathrm{~km}$ in the sunward direction, and probably twice as far (Larson and Sekanina, 1984; Sekanina and Larson, 1984). This disparity could be improved significantly be choosing mean particle densities smaller than the $2.8 \mathrm{~g} \mathrm{~cm}^{-3}$ which Delsemme and Combi suggest. Perhaps most important, Delsemme and Combi's discussion refers to the geometric center of photometric profiles and not to the point of greatest intensity, which is lost in the densest part of the photographic emulsion, while the profiles themselves are the result of a subtraction of the continuum (profile) from the total profile. It does no good to use a short-lived daughter such as [OI], since its parent (water) is relatively long-lived, is emitted predominantly on the sunward side, and may be emitted from an extended volume of sunward-biased grains. The best gas to use would be a short-lived parent, but none are known with visible lines. A much better result for astrometric purposes is likely to be obtained by confining the observations to continuum wavelengths, where both observations and the physical processes occuring are simpler, and to a search for the point of greatest intensity. This procedure should produce the best result either from space or from the ground, although ground-based observations are complicated by the convolution with atmospheric turbulence (seeing). 


\section{Applications}

One motivation for modeling these environments has been the need to assess their interactions with flight hardware for flyby missions (in the case of Halley; similar considerations potentially apply to rendezvous missions to other target comets). The following sections discuss the adaptation of the environment models needed for such purposes, and review their consequences for both spacecraft and the experiments they carry. Because GIOTTO's approach to Halley is the closest, it encounters the most severe environment and thus provides the majority of the sample applications cited.

\subsection{Adaptation of THE Models}

For the gas and dust, the respective discussions in Sections 6 and 7 provide rather complete descriptions of the environments in terms which are independent of interaction with a spacecraft or other observer. The application of these model descriptions to spacecraft engineering considerations is achieved by the development of expressions for flux and fluence, as incident on a spacecraft surface. Let the surface have velocity $\mathbf{V}_{s}$ $\left(\mathrm{m} \mathrm{s}^{-1}\right)$ with respect to the cometary nucleus, and outward normal unit vector $\mathbf{u}_{s}[$ ?] (each of these quantities may of course vary with time as described by the spacecraft trajectory and attitude). Using $n$ and $\mathbf{V}$ to represent the local differential dust concentration $\left(\mathrm{m}^{-3}\right.$ size $\left.^{-1}\right)$ and cometocentric velocity $\left(\mathrm{m} \mathrm{s}^{-1}\right)$ for particles of radius $a$, the differential number flux $\left(\mathrm{m}^{-2} \mathrm{~s}^{-1}\right.$ size $\left.^{-1}\right)$ is

$$
j_{N}=n\left|\mathbf{V}_{s}-\mathbf{V}\right|,
$$

when $\mathbf{u}_{s}$ is parallel to $\left(\mathbf{V}_{s}-\mathbf{V}\right)$; cf. Section 7.4. For surfaces with other orientations, this flux may be multiplied by the projection factor $\left(\mathbf{V}_{s}-\mathbf{V}\right) \cdot \mathbf{u}_{s} /\left|\mathbf{V}_{s}-\mathbf{V}\right|$ when the factor is positive, or by zero for a surface not exposed to the incident dust particle beam. For some purposes it is also useful to consider the incident area flux $\left(\mathrm{s}^{-1}\right.$ size $\left.^{-1}\right)$, mass flux $\left(\mathrm{kg} \mathrm{m}^{-2} \mathrm{~s}^{-1}\right.$ size $\left.^{-1}\right)$, momentum flux $\left(\mathrm{N} \mathrm{m}^{-2}\right.$ size $\left.^{-1}\right)$, and energy flux $\left(\mathrm{W} \mathrm{m}^{-2} \mathrm{size}^{-1}\right)$, which can be expressed as $\pi a^{2} j_{N}, m j_{N}, m\left|\mathbf{V}_{s}-\mathbf{V}\right| j_{N}$, and $(m / 2)\left(\mathbf{V}_{s}-\mathbf{V}\right) 2 j_{N}$ for spherical particles of mass $m=(4 \pi / 3) \rho a^{3}$. Recognizing that a variety of processes can indeed occur in the dust-surface interaction (cf. Section 10.2), these expressions nevertheless provide the correct order-of-magnitude for the transfer of area, mass, etc., to the surface, even though in detail they embody some unrealistic assumptions (e.g., that the impacting particles are retained on the surface without overlap, distortion, recoil. etc.). For example, the momentum transfer can involve a velocity-dependent multiplication factor, as discussed by McDonnell et al. (1984). For some purposes the quantity

$$
J_{N}=\int_{a_{1}}^{a_{2}} \mathrm{~d} a j_{N},
$$

which specifies the interval number flux $\left(\mathrm{m}^{-2} \mathrm{~s}^{-1}\right)$ for the size range $a_{1}<a<a_{2}$, or an integral number flux for particles larger than $a_{1}$ (same as Equation (10.2), but with $a_{2}=\infty$ ), can be useful.

A measure of the total exposure of a surface to the incident dust, in a time interval 
$t_{1}<t<t_{2}$ is provided by the fluence $\left(\mathrm{m}^{-2} \operatorname{size}^{-1}\right.$ and $\mathrm{m}^{-2}$, in the differential and integral forms) using

$$
f_{N}=\int_{t_{1}}^{t_{2}} \mathrm{~d} t j_{N}
$$

and

$$
F_{N}=\int_{t_{1}}^{t_{2}} \mathrm{~d} t J_{N}
$$

These expressions (Equations (10.2) through (10.4)) all have obvious counterparts for area flux and fluence, mass flux and fluence, etc. (cf. symbols and units cited in Appendix B).

Of course these expressions specify expected values as predicted by the dust model. When the area, size and time intervals under consideration are large enough that many particles are involved, the only uncertainties which apply are those which characterize the model itself (Section 11). However when few particles are involved, as can easily be the case for an integral fluence $F_{N}$ (Equation (10.4)) of particles when the threshold radius $a_{1}$ is large, the distribution of impacts may require additional considerations. For example, the probability of impact on a surface of area $A_{s}$ is assumed to follow Poisson statistics, in which case an important result is

$$
P_{0}=e^{-A_{s} F_{N}},
$$

for the probability of no impact when the integral number fluence is given by $F_{N}$ (Equation (10.4)). Together with an assumed random distribution in impact location, such statistical considerations have important consequences for such purposes as spacecraft attitude control (cf. Section 10.2).

For the gas, similar definitions for flux and fluence apply, except that $n$ becomes the concentration of molecules of a given species (cf. Section 6), and the differential quantities appropriate for the dust are not meaningful. For the cometary radiation, expressions already presented in Section 9 for intensity, flux, etc., can be used in various applications without additional development.

Sample values for many of these derived quantities are presented in Appendix B using our Halley model for each of three flyby spacecraft.

\subsection{CONSEQUENCES FOR SPACECRAFT AND MISSIONS}

Due to Halley's retrograde orbit the relative flyby velocities of the various spacecraft encountering Halley's comet from 6 to $14 \mathrm{March}, 1986$ are very high, ranging from $68.3 \mathrm{~km} \mathrm{~s}^{-1}$ in the case of GIOTTO to $79.2 \mathrm{~km} \mathrm{~s}^{-1}$ in the case of VEGA 1 (Table I). This causes a number of problems never before encountered on space flights. As the spacecraft approach the nucleus, cometary neutrals, ions and dust particles will impact on the spacecraft walls and experiment apertures essentially at the relative flyby velocity 
(their own velocity in the comet's frame of reference is comparatively small). Dust and gas models are of crucial importance for spacecraft design and assessment of spacecraft survival. The problems are most severe for the GIOTTO spacecraft as it flies by the nucleus 6 times closer than any other, encountering 36 times higher dust and gas fluxes. Essentially three effects have to be distinguished: mechanical destruction, attitude perturbations, and production of impact-generated plasma, as illustrated in Figure 31. An obvious first consequence for mission operation is to orient the spacecraft during their flight through the Halley coma such that a minimum area is exposed to the impacting stream of cometary particles. For GIOTTO the spin axis has to be aligned with the relative velocity vector which means for the spacecraft design that the high gain antenna has to be inclined by $22^{\circ}$ and despun to permanently point at the Earth during

\section{ENVIRONMENTAL EFFECTS}

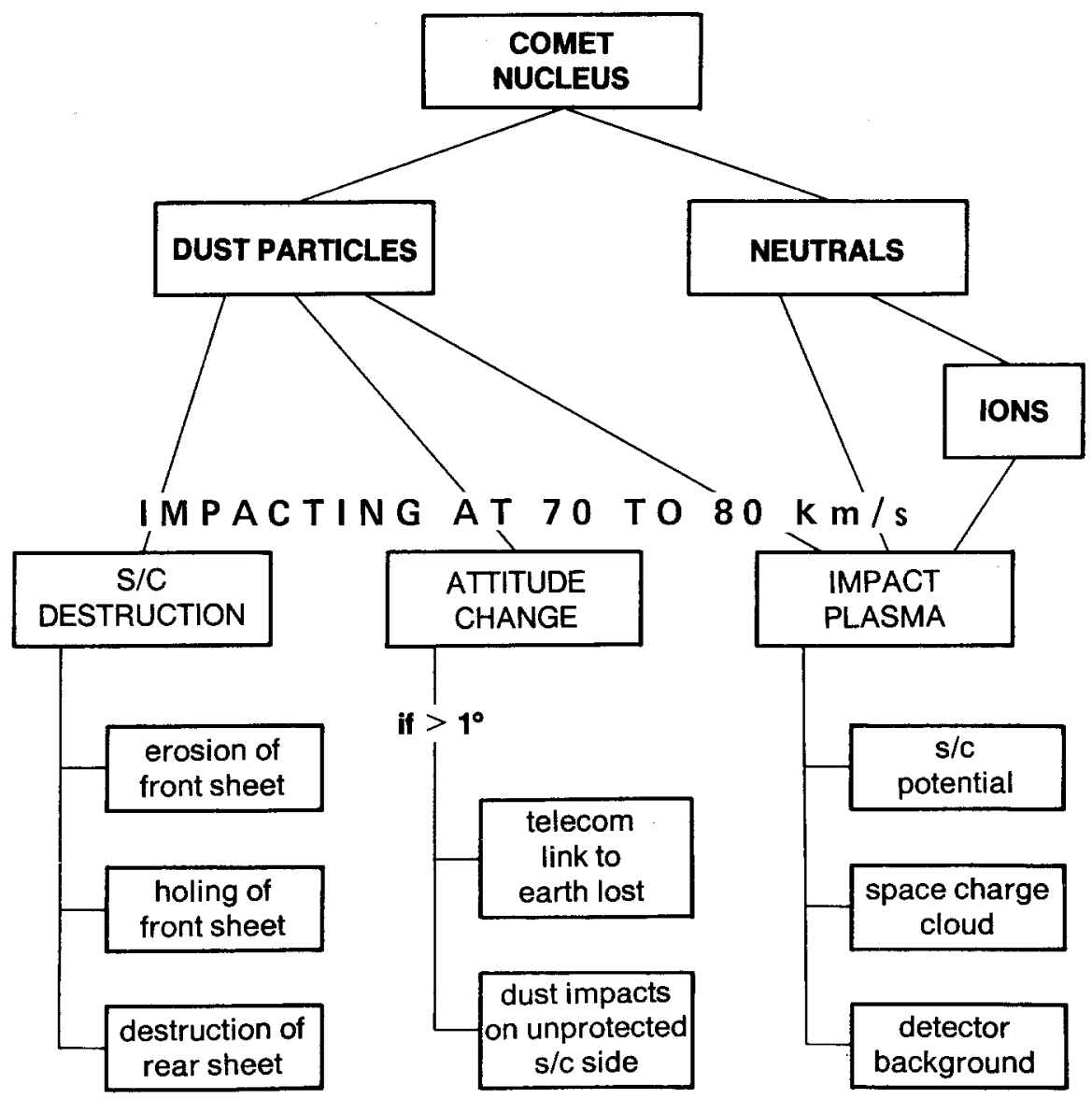

Fig. 31. Effects of cometary dust and gas environments on fast fiyby spacecraft (illustrated for GIOTTO at $P /$ Halley). 
the flyby. The flyby orientation for the VEGA spacecraft is such that the plane of the large solar cell arrays is parallel to the stream of cometary particles.

For the dust particles the integral number flux $J_{N}$ and fluence $F_{N}$ each decrease as particle radius $a$ increases. The model predictions (Appendix B) insure that for close flybys (e.g., minimum $R \lesssim 1000 \mathrm{~km}$, as for GIOTTO), a reasonable spacecraft area (e.g., a few $\mathrm{m}^{2}$ ) will receive numerous impacts of dust particles large enough to create substantial, perhaps crippling damage to any typical spacecraft wall. The damage mode can range from erosion through spallation, penetration, and structural disruption as the size of the impacting dust particle increases.

A dust particle of $0.1 \mathrm{~g}$ mass impacting at $70 \mathrm{~km} \mathrm{~s}^{-1}$ can penetrate an aluminum wall $8 \mathrm{~cm}$ thick! Considering that normal spacecraft structures have wall thicknesses of a few millimeters it can be expected that in the inner coma many thousand dust particles would penetrate the spacecraft structure and destroy the spacecraft long before the mission's scientific objectives could be fulfilled. It is, therefore, mandatory to protect the spacecraft by a shield.

Simply increasing the thickness of the leading surface is not possible as either the spacecraft cannot be protected within a reasonable shield weight or the shield weight becomes excessive for any meaningful protection. A dual-sheet bumper shield is an ideal and, in fact, the only solution. Such a shield consists of a thin front sheet and a rear sheet with some space between. The dual-sheet bumper shield works as follows: upon impact on the front sheet, the dust particle is completely vaporized, even if the front sheet is very thin. The vapor cloud expands into the empty space between the two sheets and impacts on the rear sheet, where its impact energy is distributed over a large area. It is desirable to have a large separation between the two sheets, thus distributing the impact energy over a wide area, and to make the rear sheet as thick as possible. For GIOTTO the front sheet consists of $1 \mathrm{~mm}$ thick aluminum, and the rear sheet is a sandwich made of various layers of Epoxy Kevlar, Polyurethane Foam, ML1 (Mylar), and Aluminum honeycomb with a total thickness of $7 \mathrm{~cm}$, the space between the two sheets is $23 \mathrm{~cm}$. The GIOTTO dust protection shield has been optimized and tested with dust impacts up to $8 \mathrm{~km} \mathrm{~s}^{-1}$ using a light gas gun and with dust impacts up to $68.7 \mathrm{~km} \mathrm{~s}^{-1}$ using computer simulation, and it has been concluded that this shield, which weighs only $50 \mathrm{~kg}$, can withstand $70 \mathrm{~km} \mathrm{~s}^{-1}$ impacts of dust particles up to $0.1 \mathrm{~g}$ in mass or even higher. Some shield effectiveness is sacrificed because the bumper configuration over the kick motor exhaust is not optimum and because some dust impact sensors are mounted on the outer layer itself.

To estimate the probability of spacecraft survival, three different mechanisms through which it could be destroyed have been considered:

(1) Individual very large particles, whose debris cloud may not be completely vaporized and impacts on the rear sheet, tearing it.

(2) Numerous intermediate-sized particles, which produce holes in the front sheet, so that a large fraction of its surface might disappear. A large particle might then impact directly on the rear sheet and its debris cloud would propagate into the spacecraft's interior. 
(3) A very large number of small particles, which produce craters in the front sheet, leading to its erosion. The front sheet would become thinner and thinner until holes were produced (increasing hazard 2) or until the whole front sheet disappeared.

The hazards due to these three mechanisms have been calculated and all lie in the range of a few percent for a flyby at a distance of a few hundred $\mathrm{km}$; thus mission loss is unlikely to occur because of dust impact damage.

It is not meaningful to try to improve the probability of GIOTTO's survival by using a thicker rear sheet or by increasing the space between the two sheets because the impacting dust particles also cause the spacecraft's attitude to change. A dust particle of $0.1 \mathrm{~g}$ in mass impacting on the outer edge of the bumper shield would move the center-line of the despun high-gain antenna more than $1^{\circ}$ from Earth-line, thus interrupting the $\mathrm{X}$-band telecommunication down-link. The most important science data would be lost because GIOTTO has no onboard data storage capability and no way to reestablish the attitude necessary for downlink on the timescale of the encounter. Fertig (1982), Coupé et al. (1983), and Wallis (1984) have analysed the relevant statistics and mechanics of such impact-generated torques for GIOTTO and concluded after much discussion at a final meeting in 1984 that "based on the 1984 dust model, there is a high probability $(>90 \%)$ that the GIOTTO spacecraft attitude during the flyby at $500 \mathrm{~km}$ will not be perturbed so severely $\left(>1^{\circ}\right)$ that the telecommunication link to Earth is lost." The reason is that the probability of an impact of a large dust particle on the spacecraft causing a major perturbation $\left(>1^{\circ}\right)$ is small and that the attitude variation due to the combination of several subcritical impacts is generally less than $1^{\circ}$.

Attitude changes $<1^{\circ}$, although not resulting in a catastrophic loss of the telecommunication link, would expose the GIOTTO solar cell array to impacting dust particles. Each time a single solar cell or the connection between two solar cells is destroyed a whole string of solar cells is lost and the spacecraft would soon be without power. Therefore, the front sheet of the bumper shield extends beyond the rear sheet by a few mm to provide at least some protection if the attitude changes up to $1^{\circ}$ and, secondly, GIOTTO carries four batteries which could supply full power for the duration of the coma flythrough even if the solar array were lost.

At Halley encounter velocities of $68-79 \mathrm{~km} \mathrm{~s}^{-1}$ the available kinetic energy is ample to vaporize and ionize not only most of the impacting particles themselves, but also some of the solid spacecraft materials. At $68.3 \mathrm{~km} \mathrm{~s}^{-1}$, for GIOTTO, the energy is $2.3 \times 10^{9} \mathrm{~J} \mathrm{~kg}^{-1}=24 \mathrm{eV} / \mathrm{AMU}$ for arriving ions, neutrals, and dust particles of every size and kind. For dust particles, the degree of ionization depends on the particle mass, and is $\geq 50 \%$ for particles $\leq 10^{-12} \mathrm{~g}$, and $\leq 1 \%$ for particles $\leq 10^{-7} \mathrm{~g}$ (Amandeau et al., 1984). The impacting primary atoms, molecules and ions cause the emission of secondary neutrals and ions. The ion and electron yields are $0.24-1 \%$ and $24-56 \%$, respectively, for different molecules impacting on aluminum (Rüdenauer and Steiger, 1984). This impact-generated plasma causes a space charge cloud in front of the spacecraft and a spacecraft potential, and presents a serious background problem for cometary plasma experiments.

The adverse effects of the impact-generated plasma around the spacecraft have been 
quantified using present best estimates for the Halley gas and dust production rates. The calculations show that a positive spacecraft potential of no more that a few tens of volts and a slightly higher potential in the space-charge cloud in front of the spacecraft can be expected (Parker, 1984; Thiemann, 1984). In the innermost part of the coma the density of the impact-generated plasma is several orders of magnitude higher than that of the cometary plasma. The adverse effects on detector background, however, will be limited first because the highest densities of impact-generated plasma will be above the front sheet and not at the location of the plasma sensors, and second, because the impact-generated ions can be distinguished from the cometary ions by their distinctly different velocity distributions (in the spacecraft frame of reference the distribution of cometary ions will be centered at $68 \mathrm{~km} \mathrm{~s}^{-1}$, while the distribution of the impactgenerated ions will be at rest).

The spacecraft is designed to be as conductive as possible to avoid differential charging. Gold has a much lower ion production rate than aluminum for impacting neutrals. On the other hand, gold has one of the highest ion yields for dust particle impacts. A thin $(10 \mu \mathrm{m})$ gold coating on the aluminum front sheet was, therefore, proposed (Grün and Reinhard, 1981) as the optimum solution. This solution could not be implemented as gold has a high radiative absorption to emission ratio, and the front sheet would have reached too high temperatures.

Lastly, because final targeting for GIOTTO, for both minimum distance and azimuth in the target plane, will be delayed until about a day before encounter (partly so that the terminal navigation can take advantage of ephemeris improvement based on the VEGA flybys, as in the Pathfinder concept; Münch, 1984), it might be possible to avoid the dust jets produced by active regions, using an analysis similar to that described in Section 8 , but based on the latest Halley observations. As shown by Sekanina and Larson (1984), the relation between the approach velocity and cometary spin vectors is favorable for such avoidance, in that dust from areas which were most active in 1910 would not be encountered by GIOTTO until a few seconds before closest approach.

For the other flyby spacecraft the problems resulting from gas and dust impacts are much less severe than for GIOTTO. The VEGA spacecraft passing the nucleus at a distance of $10000 \mathrm{~km}$ will observe the same effects as GIOTTO but at a greatly reduced level. These two spacecraft carry dual-sheet bumper shields to protect vital structural elements as well as some scientific payload units and cables. The outer sheet is $0.4 \mathrm{~mm}$ thick and is separated by some space from the spacecraft wall which acts as the rear sheet. The dust shield covers about half of the bottom part of the spacecraft, the half that is facing the stream of cometary dust particles during the flyby. The final targeting decision will include, among other considerations, the most recent assessment of the dust environment so that the risk of mission degradation from dust impact can be maintained at acceptable levels.

The two Japanese spacecraft carry no dust protection shield. Their science objectives can be completely fulfilled by remaining outside of the dust envelope. While SAKIGAKE passes Halley at a very large distance $\left(7 \times 10^{6} \mathrm{~km}\right)$, the dust model presented here was used to support the targeting decision for SUISEI. The dust model 
predicts that SUISEI is safe to flyby at a distance of $100000 \mathrm{~km}$. Considering model uncertainties, daily activity variations and jets the flyby distance was chosen to be $150000 \mathrm{~km}$ (Table I).

\subsection{CONSEQUENCES FOR EXPERIMENTS ON FLYBY SPACECRAFT}

Among the many considerations in the design of instrument hardware and operation, a major role is accorded to the environment. In addition to the possible provision of protection from impact damage, the gas and dust environments provide inputs, for in-situ experiments, to the selection of detector type, area, etc., of electronic thresholds and dynamic range, and of strategies for minimizing interference from competing processes. On GIOTTO (Reinhard, 1982) and the two VEGA spacecraft (Sagdeev, 1985), dust detection systems intentionally employ a wide variety of detection and analysis techniques to enable critical comparisons and calibrations among the resulting count rates and particle structure and composition data. Dust mass spectrometers (PIA on GIOTTO, PUMA on VEGA 1 and 2) detect impact rates in the mass range $3 \times 10^{-16}$ to $5 \times 10^{-10} \mathrm{~g}$ and analyse the chemical and isotopic composition of individual dust particles up to 110 AMU. Dust impact counters (piezo-electric elements (MSM), a capacitor impact detector (CIS) and an impact plasma detector (IPM) on GIOTTO, piezo-electric elements (SP-2), an impact plasma detector (SP-1) and a polyvinylidene fluoride detector (DUCMA) on VEGA 1 and 2) measure impact rates in the mass range $10^{-17}$ to $10^{-3} \mathrm{~g}$. The camera on GIOTTO and the VEGA spacecraft also sense dust impacts through the light flash that is produced when a dust particle hits the camera optical system. Furthermore, a thin $(100 \mu \mathrm{m})$ nickel screen in combination with a piezo-electric element and a photodiode (FOTON) is used on VEGA 1 and 2 to monitor impact rates and determine dust particle mass and density, a rearward looking photopolarimeter (OPE) on GIOTTO is used to measure the local spatial density of dust particles and their light scattering properties, and a radio science experiment (GRE) on GIOTTO measures the dust fluence along the GIOTTO trajectory (the spacecraft is slowed down by a few $\mathrm{cm}^{-1} \mathrm{~s}$ due to the dust impacts).

Mass and dynamic ranges for all these experiments were set during the experiment design stage according to earlier versions of the dust model. None of the dust experiments are, however, able to measure the dust terminal velocities which are too small compared to the flyby velocities. Also, they measure the local dust mass spectrum. Only with a dust model is it possible to determine the source dust mass spectrum. Given a point and time along the spacecraft trajectory, and values of $\beta$ and emission speed for several dust sizes (see Sections 7.2 and 7.4), the emission time, direction and flux from the nucleus' surface, corresponding to unit flux on a spacecraft dust detector, can be calculated. Thus irregular features in the detected dust profiles can easily be traced to their sources on the rotating nucleus.

The Halley gas model (Section 6) is useful in the design of molecular detection and analysis experiments. On GIOTTO, a neutral mass spectrometer (NMS) performs chemical and isotopic analysis of cometary neutrals up to 86 AMU over a large dynamic range (10 to $10^{7} \mathrm{~cm}^{-3}$ ), and four ion mass spectrometers perform chemical and isotopic 
analysis of cometary ions. A High Energy Range Spectrometer (HERS) analyses ions up to $64 \mathrm{AMU} / \mathrm{q}$ over the dynamic range $10^{-3}$ to $10^{2} \mathrm{~cm}^{-3}$, and a High Intensity Spectrometer (HIS) analyses ions up to $60 \mathrm{AMU} / \mathrm{q}$ over the dynamic range $10^{-2}$ to $10^{4} \mathrm{~cm}^{-3}$. An Implanted Ion Sensor (IIS) can detect cometary ions up to $40 \mathrm{AMU}$ even at very large distances from the comet, as its background count rate is $\left\langle 1 \mathrm{day}^{-1}\right.$. A Positive Ion Cluster Composition Analyser (PICCA) is optimized for operation very close to the nucleus where it can observe cometary ions up to $200 \mathrm{AMU}$ over a dynamic range $2 \times 10^{-4}$ to $2 \times 10^{2} \mathrm{~cm}^{-3}$. On VEGA 1 and 2 , a neutral mass spectrometer (ING) and an ion mass spectrometer (part of the PLASMAG-1 instrument package) analyse neutrals up to $80 \mathrm{AMU}$ (dynamic range $10^{-4}$ to $10^{6} \mathrm{~cm}^{-3}$ ) and $110 \mathrm{AMU}$ (dynamic range $10^{-3}$ to $10^{5} \mathrm{~cm}^{-3}$ ), respectively. Comparison of the instrument dynamic and mass ranges with Figures 13 and 14 shows that coverage throughout the encounter will be ample for all major and many minor constituents.

Spacecraft charging and impact-generated plasma are major concerns for ion mass spectrometers and plasma experiments on the GIOTTO and VEGA spacecraft. The positive spacecraft potential, and the space charge cloud in front of it, can deflect positive cometary ions, especially the lighter ones, noticeably away from experiment apertures. This deflection is coupled with deceleration which slightly distorts the ion spectrum. Other expected effects are spurious plasma created on the front shield, charge exchange of the secondary ions with the incoming cometary neutrals, and excess loading of high voltage power supplies by large secondary charged particle fluxes near instrument apertures, in particular after a dust particle impact (for a summary see Young, 1984). Assessment of the severity of all these effects was made based on the dust and gas models described here.

Another area of application is the protection of experiment apertures. Some experiments have especially designed protection devices, ranging from simple extensions of the otherwise circular dust shield on GIOTTO to individual single sheet or dual-sheet protection. Some experiments, such as the magnetometer and the photopolarimeter on GIOTTO, are mounted in safe positions in the particle 'shadow' of the spacecraft. As described by Keller et al. (1981, 1983), a flat deflecting steel mirror which extends beyond the edge of the dust shield allows the Halley Multicolour Camera's (HMC) Ritchey-Chrétien telescope and focal plane CCDs to remain safely in the particle 'shadow' of the spacecraft bumper shield. As the rotating camera follows the apparent motion of the comet nucleus the baffle surrounding the deflecting mirror provides some protection against the impacting dust and Agrilli and Bianchini (1983) anticipate that no more than $27 \%$ of the deflecting mirror surface will be damaged during the flyby. The cameras on the VEGA spacecraft will probably only suffer minor degradation due to dust impacts as the VEGA spacecraft fly by at a much larger distance than GIOTTO, so that optical systems tracking the comet nucleus are protected by the camera baffles much earlier.

Following the apparent motion of the comet nucleus during the flyby is not as straightforward as it may seem. Apart from the GIOTTO peculiar problem of having to employ the spin-scan principle at a moving target (due to the GIOTTO spin the 
camera 'loses' the nucleus at every rotation and has to calculate the new position of the nucleus for the next rotation) the cameras may lock on to a bright dust jet instead of the nucleus if the brightenesses are comparable. Correction by ground command is not possible, as the round trip light time is 16 minutes. A very bright jet could confuse the camera target tracking, as could multiple nuclei. The best strategy is to compare onboard the measured motion of the comet nucleus once it has been acquired with that calculated from the anticipated flyby distance/time based on astrometric observations from the ground. To make matters more difficult, the target tracking also has to cope with the spacecraft attitude perturbation from dust impacts are most severe at closest approach when the apparent motion of the nucleus is fastest.

For the Ultraviolet Imager (UVI) onboard the SUISEI spacecraft (Hirac, 1984), the environmental model was applied to establish the field-of-view and dynamic range which are suitable for viewing the atomic hydrogen corona (extent $>10^{7} \mathrm{~km}$ ) so that imaging in $\mathbf{L} \alpha$ will provide useful spatial resolution for the relevant processes from a large range of distances (from many tens of million kilometers to $150000 \mathrm{~km}$ ).

\section{Range of Uncertainties and Effects not Included}

For the several aspects of the Halley environment described in preceding chapters, the major contribution to the uncertainty comes from quantities discussed in Section 3 , namely the resonance fluorescence efficiency $\mathscr{R}$ and the Halley visual light curve. For the former we consider Figure 2, in which values for $\mathscr{R}$ are derived from gas production and magnitude data for several recent comets using Equation (3.5), and are separated by heliocentric distance $r$. If among the 44 entries in ths figure, we ignore the largest 7 and the smallest 7 values for $\mathscr{R}$, we are left with the range $2 \times 10^{-62} \mathrm{~km}^{2} \mathrm{~s}$ to $1.4 \times 10^{-60} \mathrm{~km}^{2} \mathrm{~s}$, namely a factor near $10^{ \pm 1}$ for the $\pm 1 \sigma$ values in this quantity (recall that the value adopted for $P /$ Halley in Sec. 3.1 is $\mathscr{R}=2 \times 10^{-61} \mathrm{~km}^{2} \mathrm{~s}$ ). The use of Equation (3.5) for the derivation of these values insures that their range includes the effects of various other uncertainties in the analysis, such as the exponent of $Q_{\mathrm{H}}$ in the second term of Equation (3.1), as discussed in the text of Section 3.1. The form of Equation (3.5) has the result that an uncertainty factor $10^{ \pm 1}$ for $\mathscr{R}$ leads to an uncertainty factor $3^{+1}$ in the production rates $Q_{\mathrm{H}}$. An independent contribution to the uncertainty in $Q_{\mathbf{H}}$ is provided by the Halley visual magnitude, and an appreciation of this contribution can be derived from Figure 3. If we estimate a total range of 3 mag for Halley (and this is consistent with other, preperihelion data as well), the result (from Equation (3.5)) is an additional \pm 0.3 in $\log Q_{\mathrm{H}}$. The combination of these two sources leads to a total uncertainty estimate for $Q_{\mathrm{H}}$ of a factor nearly $4^{ \pm 1}$.

Because many of the model results (e.g., gas and dust concentration, flux and fluence) are proportional to $Q_{\mathrm{H}}$, the uncertainty factor $4^{ \pm 1}$ derived above as a $\pm 1 \sigma$ estimate must be applied to such results as well. Several other uncertainty sources are potential contributors also. These include the Halley nuclear radius $R_{N}$ and mass $M_{N}$ (very poorly known, as discussed at the end of Section 4.2), the gas composition ratio $F_{\text {TW }}$ (Section 3.2), and the dust-to-gas ratio by mass $\mu$ (Section 3.2). However, if we 
introduce alternate values for these quantities (which values are consistent with our judgment of reasonable ranges) into numerical models governed by the relevant equations of Sections 5 through 7, we find that the results are affected only modestly, e.g., perhaps a factor 1.5 in outflow velocities. We conclude that these uncertainty sources do not increase the overall uncertainty in the results beyond that already attributed to $Q_{\mathrm{H}}$.

For the gas environment (Section 6), then, the factor $4^{ \pm 1}$ derived for $Q_{\mathrm{H}}$ should be appropriate as a $\pm 1 \sigma$ estimate for the concentrations of $\mathrm{H}$ and of the other major species whose spatial distributions have been observed in cometry comae (i.e., those in Table VIII). A modestly larger uncertainty may be appropriate for other species (e.g., those in Figure 13, but not in Table VIII), whose distributions are inferred primarily from theoretical considerations (Section 6.2).

For the dust, four additional considerations pertain to uncertainty estimates, and they resist easy quantification. First, the dust particle density has been modeled using Equation (7.8) and the parameter values in Appendix A. Actually, as discussed in Section 7.3, the dust grains are likely to be heterogeneous and irregular, perhaps as suggested by Figure 22, so that the actual relations among size, mass, and the parameter $\beta$ (Equation (7.7)) can be rather complex. Second, the dust distribution function, for which the model uses a form similar to Equation (7.5), is simple because enough information exists (as in Section 7.2) to fix only a few of its properties. It is possible that alternate forms might be found which provide equally good fits to the radiative and dynamical data, but for which the mass normalization, employed in Equation (7.12), could be quite different (see, for example, the very different distribution function used in prior models, as given by Reinhard, 1979, and Divine, 1981a). In this case values for differential, interval and integral concentrations, fluxes and fluences (as in Section 10.1) could be significantly affected. Third, irregular variations of dust production with time and with location on the nucleus' surface, as exemplified by the results in Section 8, can introduce significant departures of the actual dust distribution from those predicted by the smoothly varying model. Fourth, if the dust grains become electrically charged in the plasma environment, the convection electric fields can affect their trajectories, particularly for the smallest particles in the model distribution (Wallis and Hassan, 1983; Horanyi and Mendis, 1985). Potential users of the models must be prepared for the likely reality of all four of these considerations.

\section{Summary}

Numerical models have been completed for the dust, gas and radiation environments at comet $P /$ Halley, in support of spacecraft and mission design for flybys in 1986 . These models find a common basis in our theoretical understanding of cometary physics, as derived from astronomical observations of several recent comets, with particular emphasis on the prediction of Halley's activity from its magnitude in prior apparitions. Our selection of a set of parameter values for the nucleus, and for the properties of gas and dust which it generates, has been combined with several simplifying assumptions, leading to significant conclusions in three major areas. 
(1) A spacecraft which approaches Halley's nucleus to within $500 \mathrm{~km}$ in early March, 1986, will find there a gas concentration of the order of $4 \times 10^{14} \mathrm{~m}^{-3}$, dominated by $\mathrm{H}_{2} \mathrm{O}$ with 10 to $20 \%$ of other parent molecules (e.g., $\mathrm{NH}_{3}, \mathrm{CS}_{2}, \mathrm{CO}_{2}$ ). An inverse square dependence of concentration on cometocentric distance is reasonable for distances less than the parent scale lengths (cf. Table VIII), beyond which photodissociation products become dominant. At distances exceeding a few tenths of $\mathrm{Gm}$, atomic hydrogen (in two components at 8 and $20 \mathrm{~km} \mathrm{~s}^{-1}$ ) becomes the primary gaseous species. Departures from spherical symmetry are small for all gas distributions.

(2) On the average, a spacecraft which approaches Halley's nucleus to within $500 \mathrm{~km}$ in early March, 1986, will find there a dust mass concentration of the order of $3 \times 10^{-11} \mathrm{~kg} \mathrm{~m}^{-3}$, of which about $50 \%$ will be found in particles of mass exceeding $1 \mathrm{mg}$. For reasonable values of area and velocity for a flyby spacecraft, collisions with such particles can have serious consequences, including impact damage and attitude perturbation, corresponding to a predicted integral number fluence near $1 \mathrm{~m}^{-2}$. An inverse square dependence of concentration and flux (simple inverse for fluence) on cometocentric distance is reasonable for distances up to about $10^{5} \mathrm{~km}$, where the largest dust envelopes occur (sunward of the nucleus). However, irregular variations of the dust environment with time and direction (along a spacecraft trajectory) are probably large (easily an order of magnitude).

(3) Gas and dust radiation intensities, respectively up to about $10 \mathrm{kR}$ in line emission (for $\mathrm{L} \alpha$ ), $4 \mathrm{~W} \mathrm{~m}^{-2} \mu \mathrm{m}^{-1} \mathrm{sr}^{-1}$ in the visible (at $0.6 \mu \mathrm{m}$ ) and $0.3 \mathrm{~W} \mathrm{~m}^{-2} \mu \mathrm{m}^{-1} \mathrm{sr}^{-1}$ in the infrared (at $15 \mu \mathrm{m}$ ) from spacecraft perspectives are similar to those observed from Earth, but of course much greater flux and linear resolution will be available to the spacecraft detectors. Radial, directional and temporal dependences will mimic those of the responsible environments; in particluar radiation from the dust may exhibit considerable inhomogeneity, and the nucleus may or may not have enough contrast to be easily visible through the dust.

For additional detail the reader is referred to the relevant sections of the preceding text. He should also be aware that major improvements to these models (perhaps even complete abandonment and replacement) resulting from the spacecraft encounter data are possible, if not likely.

\section{Acknowledgments}

The authors thank their colleagues on WG-1, namely J. Fertig, R. Hellmich, K. Richter, G. H. Schwehm, and T. Yamamoto, for their help with various aspects of the environments described here. The research described in this paper was carried out by the Universities of California in San Diego, of Arizona, of Michigan, and by the Jet Propulsion Laboratory, California Institute of Technology, and was jointly sponsored by the European Space Research and Technology Center of the European Space Agency and MPI-Kernphysik and MPI-Aeronomie of the Max-Planck Gesellschaft, Federal Republic of Germany, through an agreement with the National Aeronautics and Space Administration. 


\section{Appendix A. Parameters and Assumptions for Model HALLEY 85-4}

To enable the numerical computation of modeled quantities for Halley's gas and dust environments, we require that the general discussions and conclusions presented in the foregoing review sections be narrowed to a specific set of parameter values and assumptions, as described in this appendix.

Tables XII through XVI collect most of the parameter values which comprise the current model, which carries the label HALLEY 85-4 to distinguish it from others in the literature. For each quantity in these tables we specify a symbol, value, units, and

\section{TABLE XII}

Constants for the dust and gas environments in model HALLEY 85-4

\begin{tabular}{lll}
\hline Quantity & Symbol, value, units & Equation \\
\hline Solar gravitational constant & $G M_{\odot}=1.32718 \times 10^{20} \mathrm{~m}^{3} \mathrm{~s}^{-2}$ & 2.1 \\
Solar luminosity & $L_{\odot}=3.83 \times 10^{26} \mathrm{~W}$ & 9.5 \\
Solar apparent visual magnitude & $m_{\odot}=-26.8$ & 3.1 \\
Astronomical unit & $r_{0}=1.496 \times 10^{11} \mathrm{~m}$ & 3.2 \\
Speed of light & $c=3.0 \times 10^{8} \mathrm{~m} \mathrm{~s}^{-1}$ & 7.7 \\
Boltzmann's constant & $k=1.3806 \times 10^{-23} \mathrm{~J} \mathrm{~K}^{-1}$ & 5.2 \\
Stefan-Boltzmann constant & $\sigma=5.67 \times 10^{-8} \mathrm{~W} \mathrm{~m}^{-2} \mathrm{~K}^{-4}$ & 9.9 \\
\hline
\end{tabular}

TABLE XIII

Parameters for the nucleus in model HALLEY 85-4 (cf. Section 4)

\begin{tabular}{lll}
\hline Quantity & Symbol, value, units & Equation \\
\hline Radius & $R_{N}=3000 \mathrm{~m}$ & 3.1 \\
Mass & $M_{N}=1.13 \times 10^{14} \mathrm{~kg}$ & 4.1 \\
Density & $\rho_{N}=1000 \mathrm{~kg} \mathrm{~m}^{-3}$ & 4.1 \\
Geometric albedo & $p_{N}=0.06$ near $\lambda=0.5 \mu m$ & 3.1 \\
Bond albedo & $A_{N}=0.12$ & 9.22 \\
Infrared emissivity & $\varepsilon_{N}=1.0$ & 5.5 \\
Surface source function coefficients & $A_{0}=0.07958 \mathrm{sr}^{-1}$ & 7.15 \\
& $A_{3}=0.04775 \mathrm{sr}^{-1}$ & 7.15 \\
\hline
\end{tabular}

an equation number which serves as a pointer to discussion in the text (commonly the first appearance of the cited symbol). Most entries for the constants in Table XII are adopted from Allen (1973), after conversion to SI units. Further, Sections 44 and 82 of Allen (1973) provide one source of formulae and data from which the distribution of solar intensity or flux with wavelength (cf. $f_{\odot}$ in Section 9.3) may be evaluated.

For Halley's nucleus, the orbital elements, parameters for nongravitational acceleration, and rotational quantities are found in Table II (solution 1), Section 2.1 (following Equation (2.2)), and Table IV, respectively. Table XIII includes additional parameters for the nucleus, as used in the present models. As discussed in Section 4.2, most values 
TABLE XIV

Parameters for cometary gas, and for the semi-empirical photometric theory, in model HALLEY 85-4 (cf. Sections 3,5 , and 6 )

\begin{tabular}{lll}
\hline Quantity & Symbol, value, units & Equation \\
\hline Ratio, total to water, by number & $F_{T W}=1.2$ & 3.7 \\
Mean molecular mass & $M_{g}=3.707 \times 10^{-26} \mathrm{~kg}$ & 5.2 \\
Ratio of specific heats & $\gamma=4$ & 5.1 \\
Water ice sublimation latent heat & $L=2.77 \times 10^{6} \mathrm{~J} \mathrm{~kg}^{-1}$ & 5.2 \\
Lifetime for $\mathrm{C}_{2}$ at $r_{0}=1 \mathrm{AU}$ & $\tau_{\mathrm{C}_{2}}=1.2 \times 10^{5} \mathrm{~s}$ & 3.2 \\
Resonance fluorescence efficiency & $\mathbb{R}=2.0 \times 10^{-55} \mathrm{~m}^{2} \mathrm{~s}$ & 3.2 \\
Parameters for dust-to-gas ratio by light & $\delta_{1}=0.416 \quad$ for $r<1.25$ & 3.3 \\
& $n_{1}=2.0 \quad$ for $r>1.25 \mathrm{AU}$ & \\
& $\delta_{1}=0.333 \quad$ & \\
Parameters for Halley light curve & $n_{1}=3$ & \\
& $H_{0}=5.47 \quad$ preperih. & \\
& $n_{0}=4.44 \quad$ & \\
& $H_{0}=0.335 \quad$ postperih. \\
& $n_{0}=-4.44 \quad r<0.71 \mathrm{AU}$ & \\
& $H_{0}=3.13 \quad$ postperih. & \\
& $n_{0}=3.09 \quad r>0.71 \mathrm{AU}$ & \\
\hline
\end{tabular}

in Table XIII are rather crude guesses suitable primarily for estimating orders of magnitude.

The major parameters for the gas model (Section 6) are collected in Table XIV, including those for the semi-empirical photometric theory (Section 3.1) and for Halley's visual light curve (Section 3.2). The values for $F_{\mathrm{TW}}$ and $M_{g}$ are consistent with a composition having $83.3 \% \mathrm{H}_{2} \mathrm{O}$ and $16.7 \%$ molecules of mean molecular mass $44 \mathrm{AMU}$, by number, in the initial sublimation products. Section 6 describes more detailed composition considerations, including a simple scale length model for the concentration of a few species (Section 6.3 and Table VIII), and, alternately, a more detailed model as described in Section 6.4 (and Figures 13 through 19). Sample results for several intermediate model quantities (including gas production $Q_{g}$ and terminal velocity $u_{T}$ as functions of heliocentric distance $r$ ) are collected in Table V, using the photometric and hydrodynamic models of Sections 3 and 5.

The dust model includes two particle kinds, as indicated by the three-to-one ratio of the first two entries in Table XV. They differ only in the dependence of $Q_{\mathrm{pr}}$ (cf. Equation (7.7)) on particle radius $a$, as given in Table XVI, where properties for absorbing grains are modeled using magnetite spheres, and for dielectric grains are modeled using olivine spheres (results of G. Schwehm and M. Hanner, private communication). All other properties are identical for the two dust populations, as evaluated using the remaining entries in Table XV, and the scattering function in Figure 20.

The parameter values collected in Tables XII through XVI have been used as inputs to computer programs which implement most of the equations of Sections 2 through 7 , resulting in the numerical model HALLEY 85-4 of dust environments, which can be evaluated for the conditions of spacecraft flybys (Table I and Section 10.1). Because 
TABLE XV

Parameters for cometary dust in model HALLEY 85-4 (cf. Section 7)

\begin{tabular}{lll}
\hline Quantity & Symbol, value, units & Equation \\
\hline $\begin{array}{ll}\text { Dust-to-gas mass production ratios } \\
\text { absorbing magnetite }\end{array}$ & \\
dielectric olivine & $\mu_{1}=0.15$ & 7.12 \\
Density function parameters & $\mu_{2}=0.05$ & 7.12 \\
& $\rho_{0}=3000 \mathrm{~kg} \mathrm{~m}^{-3}$ & 7.8 \\
& $\sigma_{0}=2200 \mathrm{~kg} \mathrm{~m}^{-3}$ & 7.8 \\
Distribution function parameters & $a_{2}=2.0 \mu \mathrm{m}$ & 7.8 \\
& $a_{0}=0.1 \mu \mathrm{m}$ & 7.5 \\
& $M=12.0$ & 7.5 \\
& $N=4.2$ & 7.5 \\
Specific heat & $a_{3}=0.01 \mathrm{~m}$ & 7.12 \\
Geometric albedo & $g_{0}=1.075 \times 10^{12} \mathrm{~kg}^{-1} \mathrm{~m}^{-1}$ & 7.5 \\
Single scattering albedo & $C=800 \mathrm{~J} \mathrm{~kg} \mathrm{~K}^{-1}$ & 5.12 \\
& $p=0.03 \quad \mathrm{near} \lambda=0.5 \mu \mathrm{m}$ & 7.2 \\
\hline
\end{tabular}

TABLE XVI

Radiation pressure efficiency for cometary dust in model HALLEY 85-4 (cf. Section 7)

\begin{tabular}{cll}
\hline $\begin{array}{l}\text { Particle } \\
\text { radius } \\
a(\mu \mathrm{m})\end{array}$ & \multicolumn{2}{c}{ Radiation Pressure efficiency, $Q_{\mathrm{pr}}$} \\
\cline { 2 - 3 } & Absorbing magnetite & Dielectric olivine \\
\hline 0.10 & 1.54 & 0.30 \\
0.16 & 2.0 & 0.69 \\
0.22 & 2.1 & 0.94 \\
0.40 & 1.79 & 1.20 \\
0.63 & 1.53 & 1.12 \\
1.0 & 1.38 & 0.96 \\
2.0 & 1.19 & 0.75 \\
5.0 & 1.07 & 0.63 \\
10.0 & 1.00 & 0.64 \\
20.0 & 1.00 & 0.70 \\
50.0 & 1.00 & 0.82 \\
100 & 1.00 & 1.00 \\
1000 & 1.00 & 1.00 \\
10000 & 1.00 & 1.00 \\
\hline
\end{tabular}

many of the chapters in this review discuss more than a single level of complexity for the various environmental descriptions, the computer program restricts its evaluations to the following simplifying assumptions:

(1) Halley's position is computed from the two-body Keplerian relations which satisfy Equation (2.1), assuming $R_{p}=g_{n}=0$, and elements as given in Table II. Thus planetary and nongravitational perturbations are ignored. 
(2) Because the light curve represented by the parameter values in Table XIV is a composite for several apparitions, the photometric theory (Equation (3.3) through (3.7)) has been evaluated using $\Phi_{\mathrm{s}}=\Phi_{\pi}$ for all values of $r$ (rather than for the circumstances of the 1910 apparition, for example) to provide the gas production rates in Table V.

(3) The equations of Section 5 have been used to compute steady-state, isotropic terminal velocities for both gas and dust, using the size distribution for the latter (Equation (7.5) and parameter values in Table XV) and a spherical nucleus (parameters in Table XIII), including cometary gravitation. The computation was made independently for each row of $\mathrm{Table} \mathrm{V}$, which illustrates the heliocentric position dependence of a few input and derived quantities. A constant upper limit $a_{3}=0.01 \mathrm{~m}$ is used for the dust size distribution at all times.

(4) For the remainder of the computation the nucleus is treated as a point source from which the gas and dust depart at isotropic terminal velocities, but whereas the gas flux is isotropic, the dust flux depends smoothly on angle, peaking in the subsolar direction at four times the minimum for the anti-solar direction (Equation (7.15) and parameters $A_{0}$ and $A_{3}$ from Table XIII).

(5) For each of several dust particle sizes, an iteration is employed to find the two-body Keplerian trajectory which connects the target point (i.e., the spacecraft) to the emission point (i.e., the nucleus' position at an earlier time), under the influence of solar gravity and radiation pressure (ignoring cometary and planetary gravitation), for corresponding values of terminal velocity $V$ and parameter $\beta$ (Equation (7.7)). For short times of flight the fountain model (Equation 7.9 through 7.14) is used to bypass the iteration.

(6) The prior time and direction of emission are used to evaluate the dust production functions $q$ and $f_{s}$, and the Jacobian $J_{c}$, which determine the present local concentration (Equation (7.12) through (7.15)).

(7) Flux and fluence are valuated for surfaces assumed normal to the relative dust-spacecraft velocity vector (Equation (10.1) through (10.4)). For spacecraft trajectory segments which cross dust particle envelopes (where differential concentration has an artificial infinity), an alternate estimate is made for the fluence contribution (in lieu of Equation (10.3)). The unavoidable effects of discrete numerical sampling (in both time and dust particle size) include irregular and unreliable flux values near the dust envelopes. 
画

竞

ơ

谣

E

通

号

$\vec{a}$

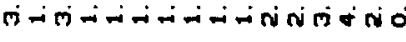

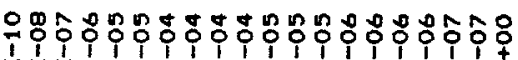

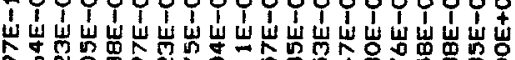

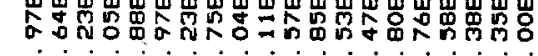
- - - +

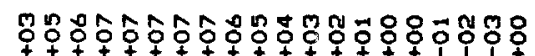

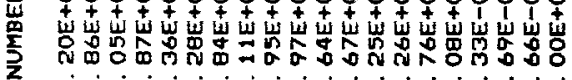

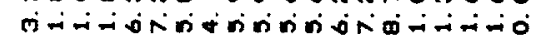

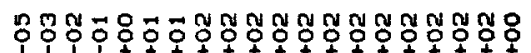

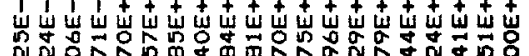
त几

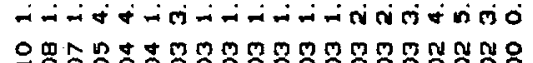

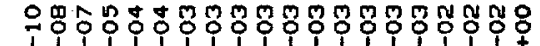

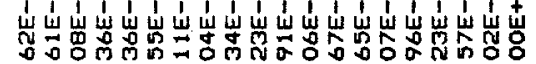

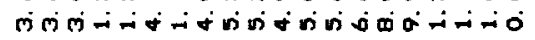

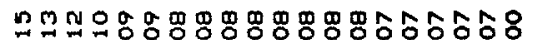

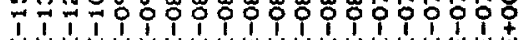




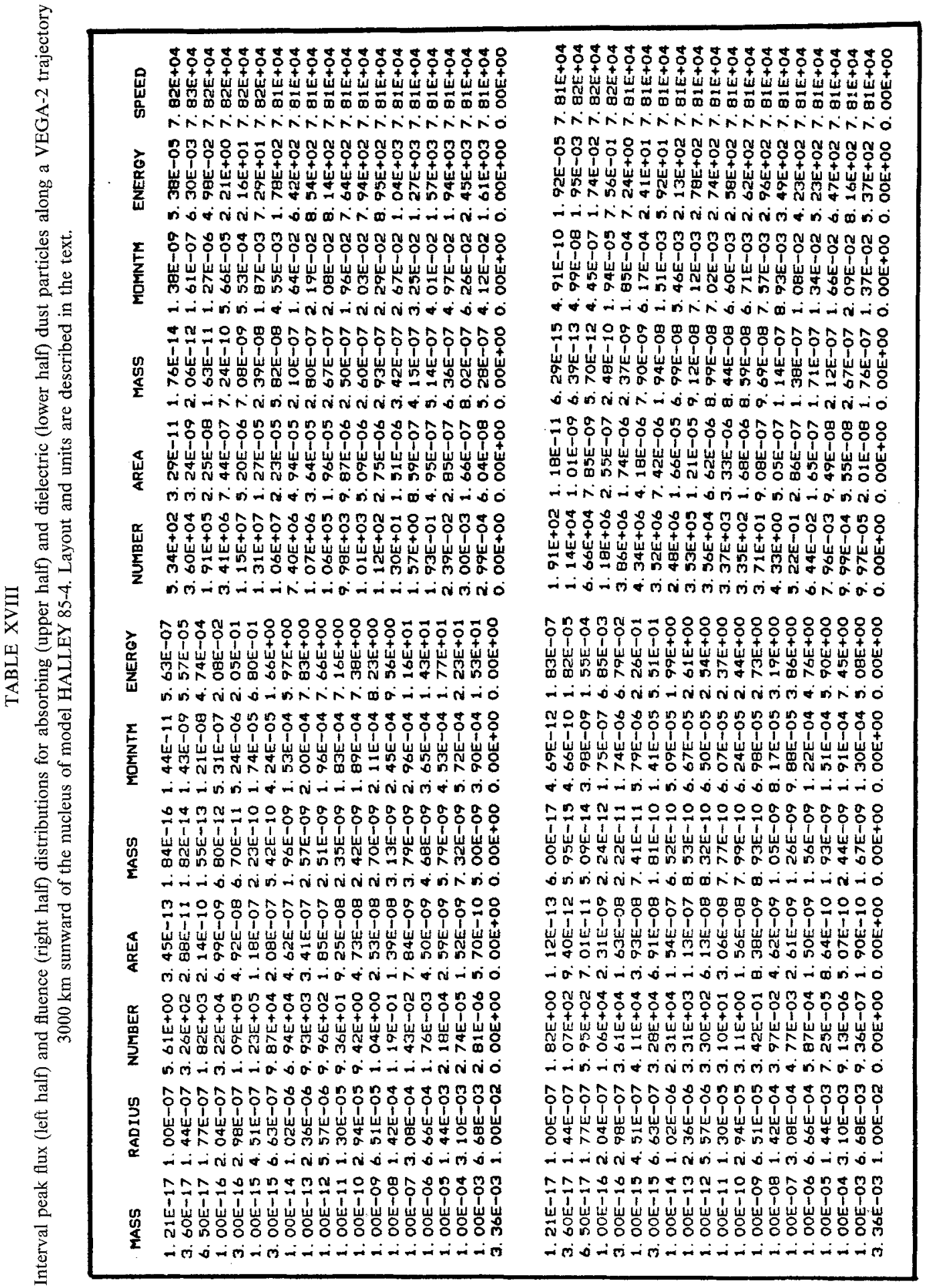




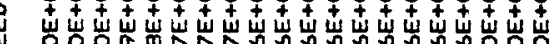

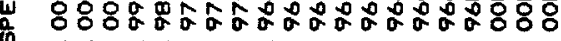

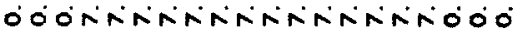

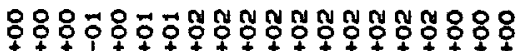

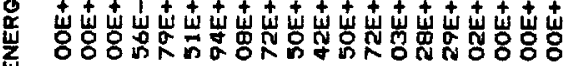

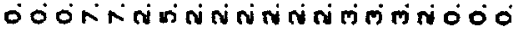

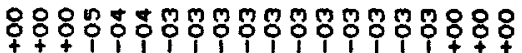

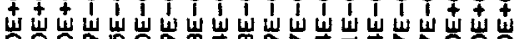

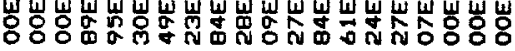

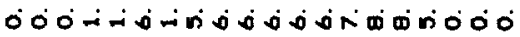

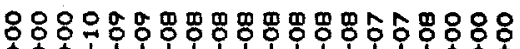

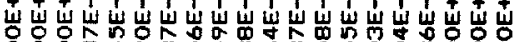

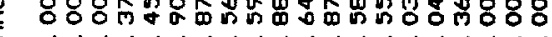

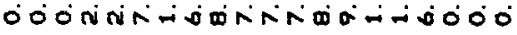

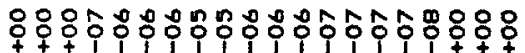

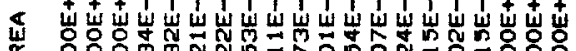
㟧

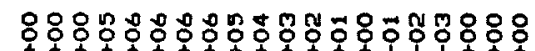

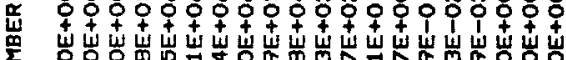

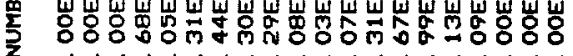

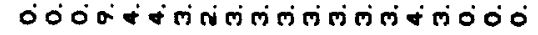

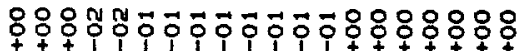

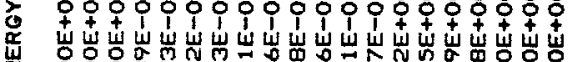
嵌

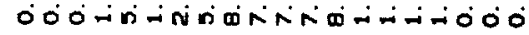

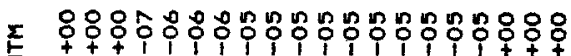

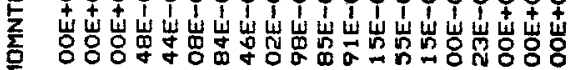

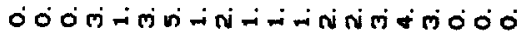

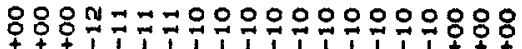

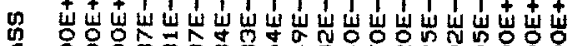

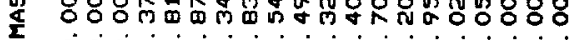

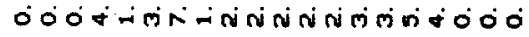

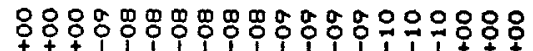

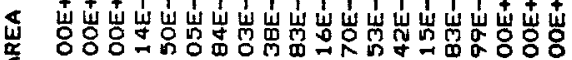

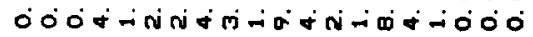

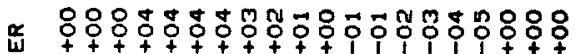

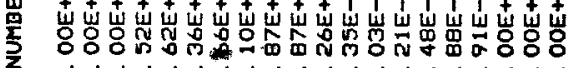

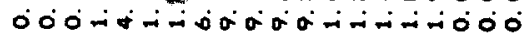

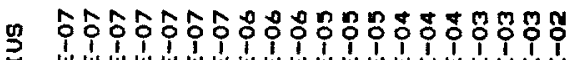

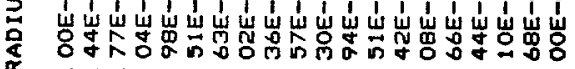

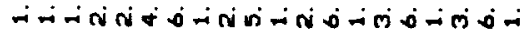

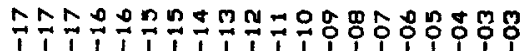

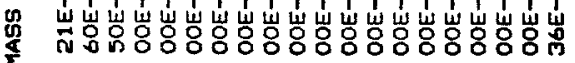

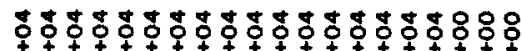

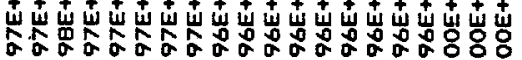

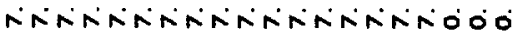

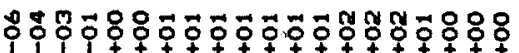

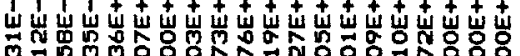

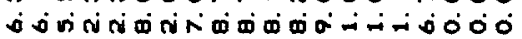

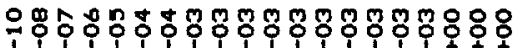

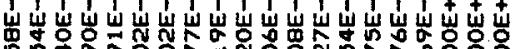

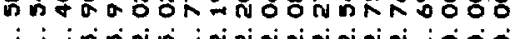
- - - -

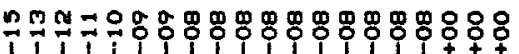

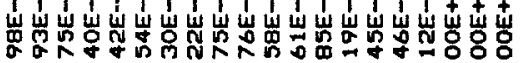

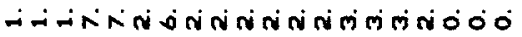

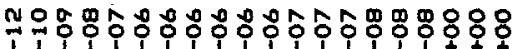

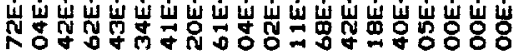

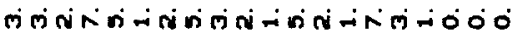

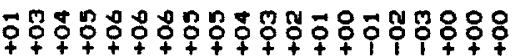

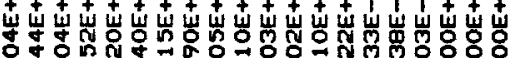
ه

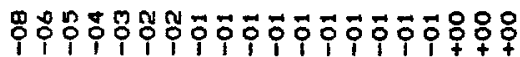

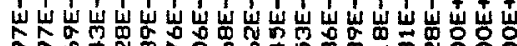

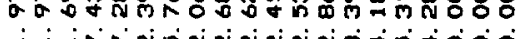

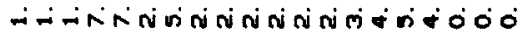

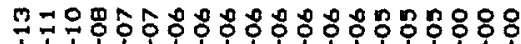

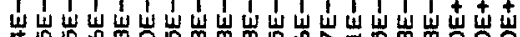

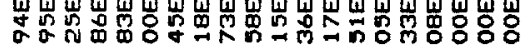

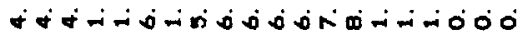

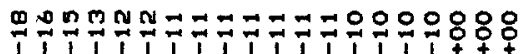

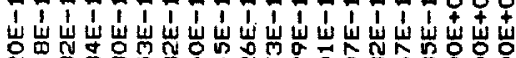

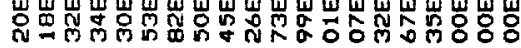

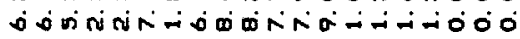

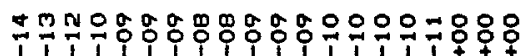

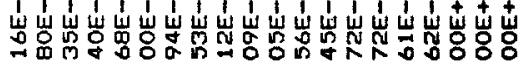

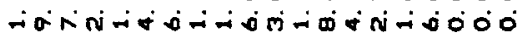

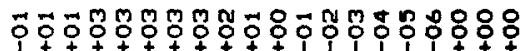

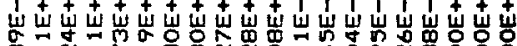

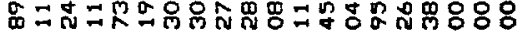

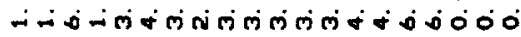

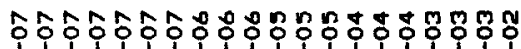

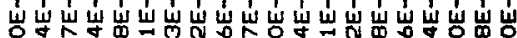

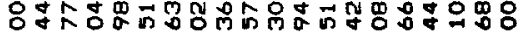

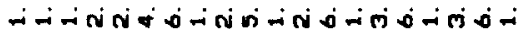

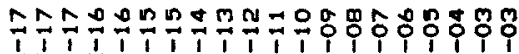

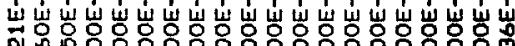

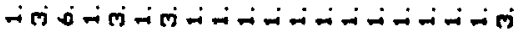




\section{Appendix B. Dust Distributions for Flyby Missions}

The parameter values and assumptions for model HALLEY 85-4 (given in Appendix A) and a dust model computer program have been applied to the spacecraft encounters listed in Table I. Sample results are presented in Tables XVII through XIX for the GIOTTO and VEGA spacecraft; neither SAKIGAKE nor SUISEI encounter any dust along their nominal trajectories in this model. In each of these three tables the 13 columns specify:

(1) threshold dust particle mass, $m(\mathrm{~kg})$,

(2) threshold dust particle radius, $a(\mathrm{~m})$,

(3) peak interval number flux, $J_{N}\left(\mathrm{~m}^{-2} \mathrm{~s}^{-1}\right)$,

(4) peak interval area flux, $J_{A}\left(\mathrm{~s}^{-1}\right)$,

(5) peak interval mass flux, $J_{M}\left(\mathrm{~kg} \mathrm{~m}^{-2} \mathrm{~s}\right)^{-1}$,

(6) peak interval momentum flux, $J_{m}\left(\mathrm{~N} \mathrm{~m}^{-2}\right)$,

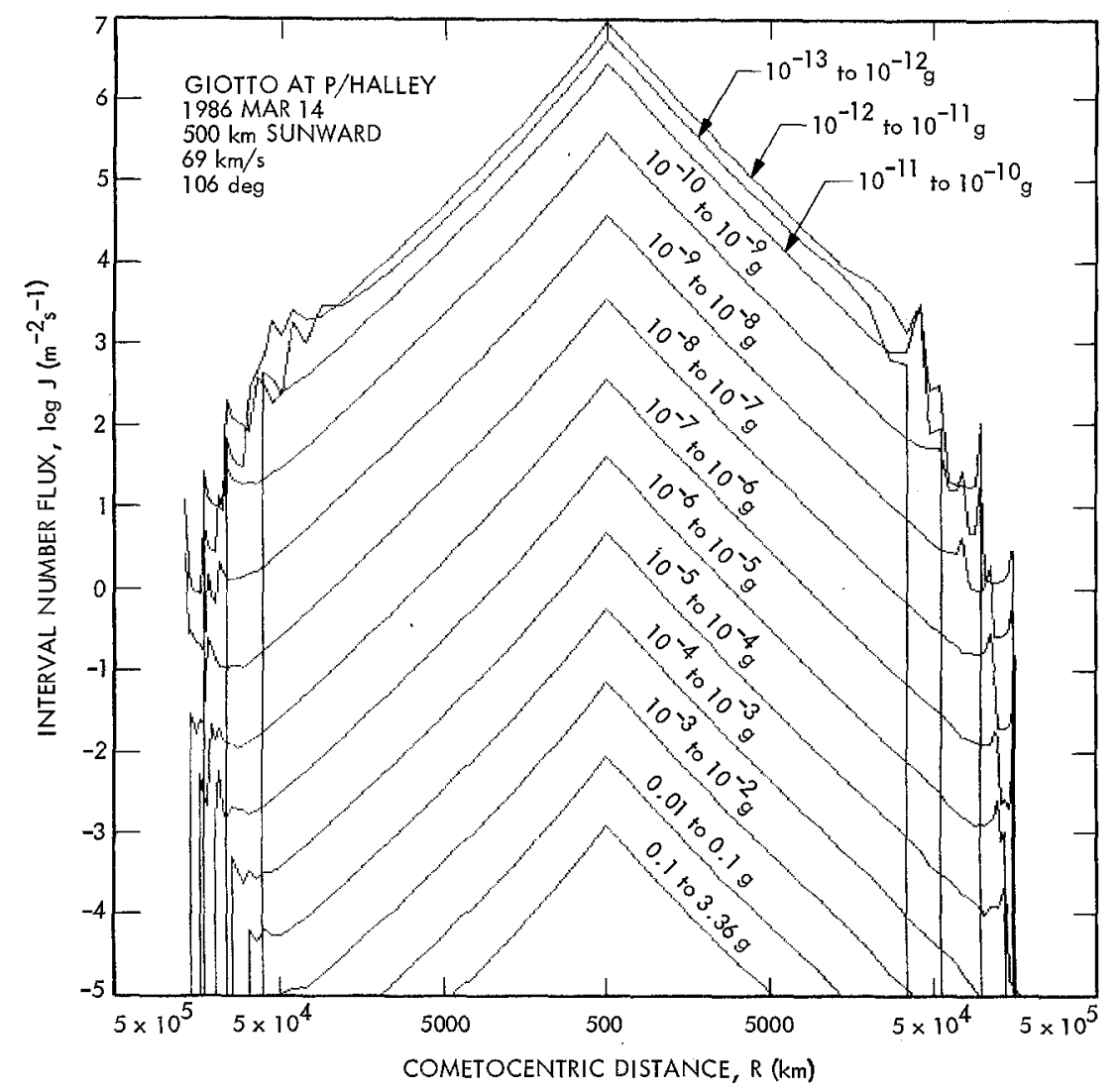

Fig. 32. Profiles of interval number flux for the GIOTTO flyby through model HALLEY 85-4. For each of the thirteen decadic intervals (in dust particle mass) shown, the central portion represents $J$ approximately proportional to $R^{-2}$ (note the logarithmic scales), whereas the irregular profile edges result from finite numerical sampling in both the time and dust size domains near the dust particle envelopes. 


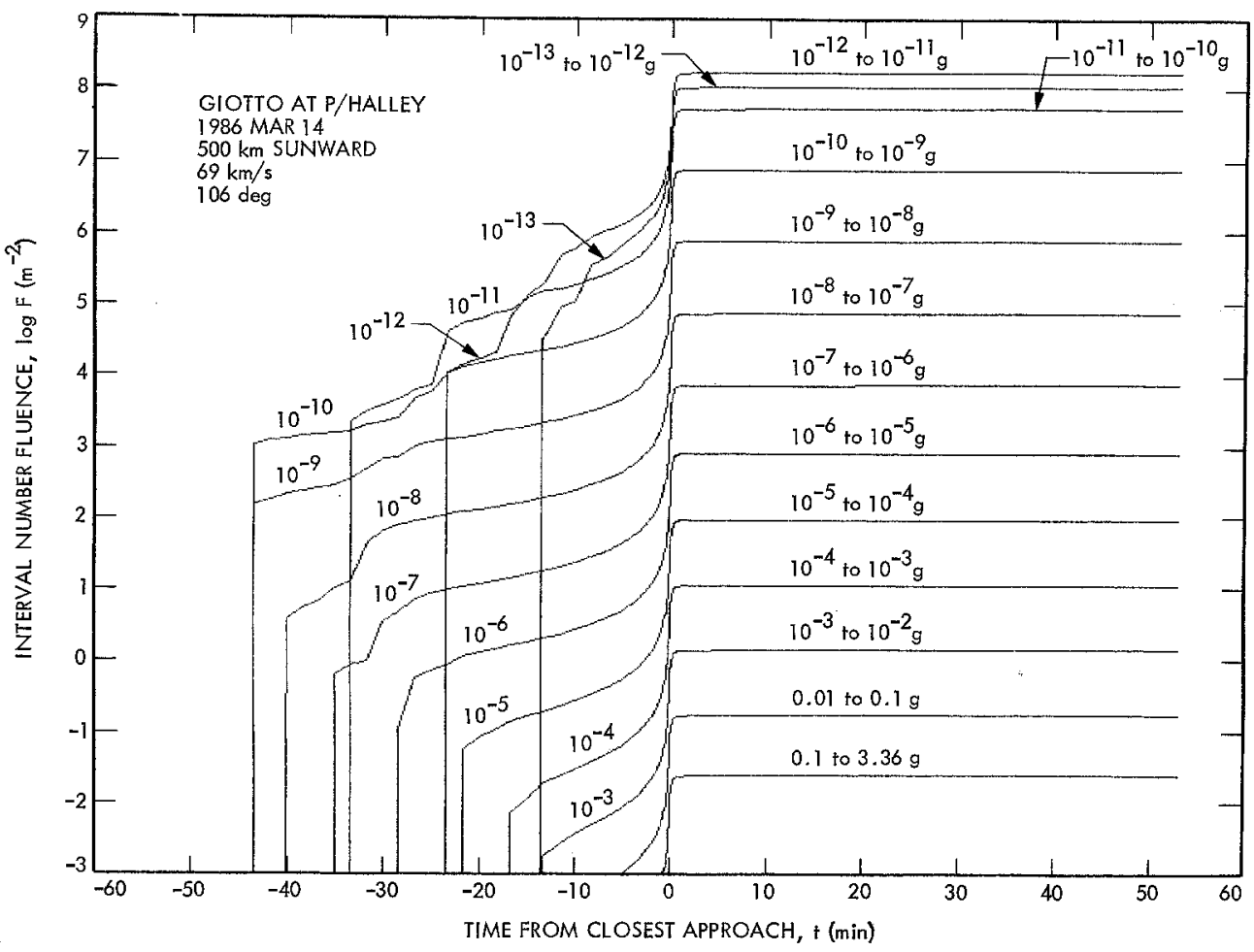

Fig. 33. Profiles of interval number fluence for the GIOTTO flyby through model HALLEY 85-4. For each of the thirteen decadic intervals (in dust particle mass) shown, the major contribution is accumulated within one minute of closest approach, whereas the first encounters with the dust envelopes are spread out over more than half an hour, with intermediate-sized particles being encountered earliest.

(7) peak interval energy flux, $J_{E}\left(\mathrm{~W} \mathrm{~m}^{-2}\right)$,

(8) interval number fluence, $F_{N}\left(\mathrm{~m}^{-2}\right)$,

(9) interval area fluence, $F_{A}(-)$,

(10) interval mass fluence, $F_{M}\left(\mathrm{~kg} \mathrm{~m}^{-2}\right)$,

(11) interval momentum fluence, $F_{m}\left(\mathrm{~kg} \mathrm{~s}^{-1} \mathrm{~m}^{-1}\right)$,

(12) interval energy fluence, $F_{E}\left(\mathrm{~J} \mathrm{~m}^{-2}\right)$, and

(13) average dust/spacecraft speed, $\left\langle\left|\mathbf{V}_{s}-\mathbf{V}\right|\right\rangle\left(\mathrm{m} \mathrm{s}^{-1}\right)$.

Each row represents an integration over the interval between the dust threshold size at its left margin and that at the left margin of the next row down. The upper half is for absorbing particles (represented by magnetite, with label 'kind 1'), and the lower half is for dielectric particles (olivine, kind 2).

Figures 32 and 33 present sample profiles of interval number flux and fluence for the GIOTTO trajectory through model HALLEY 85-4 (cf. Table I and Appendix A). 


\section{References}

A'Hearn, M. F.: 1975, Astron. J. 80, 861.

A'Hearn, M. F.: 1978, Astrophys. J. 219, 768.

A'Hearn, M. F.: 1982, in L. Wilkening (ed.), Comets, University Arizona Press, Tucson, p. 433.

A'Hearn, M. F. and Cowan, J. J.: 1975, Astron. J. 80, 852.

A'Hearn, M. F. and Cowan, J. J.: 1980, Moon and Planets 23, 41.

A'Hearn, M. F., Hanisch, R. J., and Thurber, C. H.: 1980, Astron. J. 85, 74.

A'Hearn, M. F. and Millis, R. L.: 1980, Astron. J. 85, 1528.

A'Hearn, M. F., Thurber, C. H., and Millis, R. L.: 1977, Astron. J. 82, 518.

Aiken, A. L.: 1974, Astrophys. J. 193, 263.

Allen, C. W.: 1973, Astrophysical Quantities, third ed., Athlone Press, London.

Alpern, M.: 1978, in W. G. Driscoll and W. Vaughan (eds.), Handbook of Optics, McGraw-Hill, New York, p. $12-1$.

Altenhoff, W. J., Batrla, W., Huchtmeier, W. K., Schmidt, J., Stumpff, P., and Walmsley, M.: 1983, Astron. Astrophys. 125, L19.

Amandeau, F., de Rouvray, A., and Winkelmüller, G.: 1984, ESA SP-224, 21.

Angrilli, F. and Bianchini, G. A.: 1983, in T. I. Gombosi (ed.), 'Cometary Exploration', Central Res. Inst.

Physics, Hungarian Acad. Sci., Budapest 3, 273.

Ashihara, O.: 1978, Icarus 35, 369.

Belton, M. J. S. and Butcher, H.: 1982, Nature 298, 249.

Bessel, F. W.: 1835, Astron. Nachr. 13, 3.

Bessel, F. W.: 1836a, Astron. Nachr. 13, 185.

Bessel, F. W.: 1836b, Astron. Nachr. 13, 345.

Biermann, L., Giguere, P. T., and Huebner, W. F.: 1982, Astron. Astrophys. 108, 221.

Blamont, J.: 1983, in T. I. Gombosi (ed.), 'Cometary Exploration', Central Res. Inst. Physics, Hungarian Acad. Sci., Budapest 3, 127.

Bobrovnikoff, N. T.: 1927, Astrophys. J. 66, 145.

Bobrovnikoff, N. T.: 1931, Publ. Lick Obs. 17, 305.

Bobrovnikoff, N. T.: 1954, Astron. J. 59, 357.

Bortle, J. E. and Morris, C. S.: 1984, Sky Telesc. 67, 9.

Bowell, E.: 1982, Minor Planet Circ., No. 6841.

Breus, T. K.: 1982, Space Sci. Rev. 32, 361.

Broughton, R. P.: 1979, J. Roy. Astron. Soc. Canada 73, 24.

Brownlee, D. E.: 1978, in J. A. M. McDonnell (ed.), Cosmic Dust, J. Wiley, New York, p. 295.

Brownlee, D. E., Horz, F., Hartung, J. B., and Gault, D.: 1975, Proc. 6th Lunar Sci. Conf., 3409.

Brownlee, D. E., Tomandl, D. A., and Olszewski, E.: 1977, Proc. 8th Lunar Sci. Conf., 149.

Brunner, W. and Michel, K. W.: 1968, Mitt. Astron. Ges. 25, 220.

Campins, H., Rieke, G. H., and Lebofsky, M. J.: 1983, Nature 301, 405.

Chamberlain, J. W.: 1961, Physics of the Aurora and Airglow, Academic Press, New York.

Clarke, D.: 1971, Astron. Astrophys. 14, 90.

Combi, M. R.: 1978, Astron. J. 83, 1459.

Combi, M. R. and Delsemme, A. H.: 1980, Astrophys. J. 237, 633.

Coupé, G. M., Dean, R. M., and Lainé, R.: 1983, ESA J. 7, 15.

Crifo, J. F.: 1983, in T. I. Gombosi (ed.), 'Cometary Exploration', Central Res. Inst. Physics, Hungarian Acad. Sci., Budapest 2, 167.

Crovisier, J.: 1985, Astron. J. 90, 670.

Crovisier, J. and Encrenaz, Th.: 1983, Astron. Astrophys. 126, 170.

Cruikshank, D. P. and Brown, R. H.: 1983, Icarus 56, 377.

Delsemme, A. H.: 1973, Space Sci. Rev. 15, 89.

Delsemme, A. H.: 1982, in L. Wilkening, (ed.), Comets University Arizona Press, Tucson, p. 85.

Delsemme, A. H. and Combi, M. R.: 1983, Astrophys. J. 271, 388.

Delsemme, A. H. and Miller, D. C.: 1971, Planetary Space Sci. 19, 1229.

Delsemme, A. H. and Rud, D. A.: 1973, Astron. Astrophys. 28, 1.

Delsemme, A. H. and Swings, P.: 1952, Ann. Astrophys. 15, 1.

Delsemme, A. H. and Yeomans, D K: 1984, Bull. Am. Astron. Soc. 16, 636. 
Divine, N.: 1981a, ESA SP-174, 25.

Divine, N.: 1981b, ESA SP-174, 47.

Divine, N. and Newburn, R. L. Jr.: 1983, in T. I. Gombosi (ed.), 'Cometary Exploration', Central Res. Inst. Physics, Hungarian Acad. Sci., Budapest 2, 81.

Dobrovolsky, O. V.: 1966, Komety, Nauka, Moscow.

Dobrovolsky, O. V., Kiselev, N. N., Chernova, G. P., Tupieva, F. A., and Narizhnara, N. V.: 1980, in Halliday and B. A. McIntosh (eds.), Solid Particles in the Solar System, D. Reidel Publ. Co., Dordrecht, Holland, p. 259.

Dodd, R. T.: 1981, Meteorites, A Petrologic-Chemical Synthesis, Cambridge University Press, Cambridge.

Donn, B. and Rahe, J.: 1982, in L. Wilkening (ed.), Comets, University Arizona Press, Tucson, p. 203.

Donn, B., Daniels, P. A. and Hughes, D. W.: 1985, Bull. Am. Astron. Soc. 17, 520.

Drake, J. F., Jenkins, E. B., Bertaux, J. L., Festau, M., and Keller, H. U.: 1976, Astrophys. J. 209, 302.

Encrenaz, Th., Crovisier, J., Combes, M., and Crifo, J. F.: 1982, Icarus 51, 660.

Fanale, F. P. and Salvail, J. R.: 1984, Icarus 60, 476.

Feldman, P. D.: 1982, in L. Wilkening (ed.), Comets, University Arizona Press, Tucson, p. 461.

Feldman, P. D.: 1983, Science 219, 347.

Feldman, P. D., Takacs, P. Z., Fastie, W. G., and Donn, B.: 1974, Science 185, 705.

Fertig, J: 1982, GIOTTO Study Note, No. 28, ESOC, Darmstadt.

Festou, M. C.: 1981a, Astron. Astrophys. 95, 69.

Festou, M. C.: 1981b, Astron. Astrophys. 96, 52.

Festou, M. C.: 1984, Adv. Space Res. 4 (9), 165.

Festou, M. C., Jenkins, E. B., Keller, H. U., Barker, E. S., Bertaux, J. L., Drake, J. F., and Upson, W. L. II: 1979, Astrophys. J. 232, 318.

Finson, M. L. and Probstein, R. F.: 1968, Astrophys. J. 154, 353.

Fraundorf, P., Brownlee, D. E., and Walker, R. M.: 1982, in L. Wilkening (ed.), Comets University Arizona Press, Tucson, p. 383.

Giese, R. H.: 1980, in I. Halliday and B. A. McIntosh (eds.), Solid Particles in the Solar System, D. Reidel Publ. Co., Dordrecht, p. 1.

Giguere, P. T. and Huebner, W. F.: 1978, Astrophys. J. 223, 638.

Goldstein, R. M., Jurgens, R. F., and Sekanina, Z.: 1984, Astron. J. 89, 1745.

Gombosi, T. I.: 1985, EOS Transactions, Am. Geophys. Union 66, 33.

Gombosi, T. I., Cravens, T. E., and Nagy, A. F.: 1985, Astrophys. J. 293, 328.

Gombosi, T. I., Szego, K., Gribov, B. E., Sagdeev, R. Z., Shapiro, V. D., Shevchenko, V. I., and Cravens, T. E.: in T. I. Gombosi (ed.), 'Cometary Exploration', Central Res. Inst. Physics, Hungarian Acad. Sci., Budapest, $2,99$.

Greenberg, J. M.: 1982, in L. Wilkening, (ed.), Comets University Arizona Press, Tucson, p. 131.

Greenberg, J. M.: 1983, in T. I. Gombosi, (ed.), 'Cometary Exploration', Central Res. Inst. Physics, Hungarian Acad. Sci., Budapest 2, 23.

Grün, E. and Reinhard, R.: 1981, ESA SP-155, 7.

Grün, E., Pailer, N., Fechtig, H., and Kissel, J.: 1980, Planetary Space Sci. 28, 333.

Grün, E., Zook, H. A., Fechtig, H., and Giese, R. H.: 1985, Icarus 62, 244.

Halley, E.: 1705, Synopsis Astronomiae Cometicae, London.

Hamid, S. E.-D. and Whipple, F. L.: 1953, Astron. J. 58, 100.

Hanner, M. S.: 1980, Icarus 43, 373.

Hanner, M. S.: 1981, Icarus 47, 342.

Hanner, M. S.: 1983, in T. I. Gombosi (ed.), 'Cometary Exploration', Central Res. Inst. Physics, Hungarian Acad. Sci., Budapest 2, 1.

Hanner, M. S.: 1984a, Astrophys. J. 277, L75.

Hanner, M. S.: 1984b, Adv. Space Res. 4 (9), 189.

Hanner, M. S. and Campins H.: 1985, Icarus, in press.

Hanner, M. S., Giese, R. H., Weiss, K., and Zerull, R.: 1981, Astron. Astrophys. 104, 42.

Hanner, M. S., Aitken, D. K., Knacke, R., McCorkle, S., Roche, P. F., and Tokunaga, A. T.: 1985a, Icarus 62, 97.

Hanner, M. S., Tedesco. E., Tokunaga, A. T., Veeder, G. J., Lester, D. F., Witteborn, F. C., Bregman, J. D., Gradie, J., and Lebofsky, L.: 1985b, Icarus 64, 11.

Hanner, M. S., Knacke, R., Sekanina, Z., and Tokunaga, A. T.; 1985c, Astron. Astrophys. 152, 177. 
Hansen, J. E. and Travis, L. D.: 1974, Space Sci. Rev. 16, 527.

Haser, L.: 1957, Bull. Acad. Roy. Sci. Belgique 43, 740.

Hellmich, R.: 1981, Astron. Astrophys. 93, 341.

Hellmich, R. and Keller, H. U.: 1980, in I. Halliday and B. A. McIntosh (eds.), Solid Particles in the Solar System D. Reidel Publ. Co., Dordrecht, Holland, p. 255.

Hellmich, R. and Keller, H. U.: 1981a, Icarus 47, 325.

Hellmich, R. and Keller, H. U.: 1981b, ESA SP-174, 31.

Hirao, K: 1984, SES-TD-84-004, ISAS, Tokyo.

Hognar, W. and. Richter, N.: 1980, Isophotometric Atlas of Comets, Springer-Verlag, Berlin.

Horanyi, M. and Mendis, D. A.: 1985, Astrophys. J. 294, 357.

Horanyi, M., Gombosi, T. I., Cravens, T. E., Westphal, J. A., Schneider, D. P., Dressler, A., Schmidt, M., and Zimmerman, B. A.: 1982, IAU Circ., No. 3737.

Houpis, H. L. F., Ip, W. H., and Mendis, D. A.: 1985, Astrophys. J. 295, 654.

Huebner, W. F. and Giguere, P. T.: 1980, Astrophys. J. 238, 753.

Huebner, W. F., Giguere, P. T., and Slattery, W. L.: 1982, in L. Wilkening (ed.), Comets, University Arizona Press, Tucson, p. 496.

Hughes, D. W.: 1985, Monthly Notices Roy. Astron. Soc. 213, 103.

Ip, W. H.: 1981, ESA SP-169, 79.

Ip, W. H. and Axford, W. I.: 1982, in L. Wilkening (ed.), Comets, University Arizona Press, Tucson, p. 588.

Isobe, S., Saito, K., Tomita, K., and Maehara, H.: 1978, Publ. Astron. Soc. Japan 30, 687.

Jackson, W. M.: 1982, in L. Wilkening (ed.), Comets, University Arizona Press, Tucson, p. 480.

Jambor, B. J.: 1973, Astrophys. J. 185, 727.

Jewitt, D. C., Danielson, G. E., Gunn, J. E., Westphal, J. A., Schneider, D. P., Dressler, A., Schmidt, M., and Zimmerman, B. A.: 1982, IAU Circ., No. 3737.

Jewitt, D. and Danielson, G. E.: 1984, Icarus 60, 435.

Johnson, J. R., Fink, U., and Larson, S. M.: 1984, Icarus 60, 351.

Johnson, R. E., Lanzerotti, L. J., and Brown, W. L.: 1984, Adv. Space Res. 4 (9), 41.

Kamoun, P. G., Campbell, D. B., Ostro, S. J., Pettengill G. H., and Shapiro, I. I.: 1982a, Science $216,293$.

Kamoun, P., Pettengill, G., Shapiro, I., and Campbell, D.: 1982b, Bull. Am. Astron. Soc. 14, 753.

Kelemen, J. and Toth., I.: 1983, in T. I. Gombosi (ed.), 'Cometary Exploration', Central Res. Inst. Physics, Hungarian Acad. Sci., Budapest 3, 243.

Keller, H. U.: 1973, Astron. Astrophys. 23, 269.

Keller, H. U.: 1976, Space Sci. Rev. 18, 641.

Keller, H. U. and Meier, R. R.: 1976, Astron. Astrophys. 52, 273.

Keller, H. U. and Meier, R. R.: 1980, Astron. Astrophys. 81, 210.

Keller, H. U. and Thomas, G. E.: 1975, Astron. Astrophys. 39, 7.

Keller, H. U., Arpigny, C., Barbieri, C., Benvenuti, P., Biermann, L., Bonnet, R. M., Czes, S., Colombo, G., Cosmovici, C. B., Delanere, W. A., Huebner, W. F., Hughes, D. W., Hunt, G. E., Jamar, C., Mackay, C. D., Malaise, D., Schmidt, W. K. H., Seige, P., Whipple, F. L., and Wilhelm, K.: 1981, ESA SP-169, 105 .

Astrophys. 39, 7.

Keller, H. U., Arpigny, C., Barbieri, C., Benvenuti, P., Biermann, L., Bonnet, R. M., Czes, S., Colombo, G., Cosmovici, C. B., Delanere, W. A., Huebner, W. F., Hughes, D. W., Jamar, C., Malaise, D., Schmidt, W. K. H., Seige, P., Whipple, F. L., and Wilhelm, K.: 1983, in T. I. Gombosi (ed.), 'Cometary Exploration', Central Res. Inst. Physics, Hungarian Acad. Sci., Budapest 3, 265.

Kiang, T.: 1971, Mem. Roy. Astron. Soc. 76, 27.

Kiselev, N. N. and Chernova, G. P.: 1981, Icarus 48, 473.

Klare, G., Roser, S., Schwehm, G., and West, R.: 1984, personal communication.

Klinger, J.: 1980, Science 209, 271.

Korosmezey, A.: 1984, unpublished preprint, Central Res. Inst. Phys., Hungarian Acad. Sci., Budapest.

Kührt, E.: 1984, Icarus 60, 512.

Larson, S. M. and A'Hearn, M. F.: 1984, Icarus 58, 446.

Larson, S. M. and Sekanina, Z.: 1984, Astron. J. 89, 571, 600.

Larson, S. M. and Sekanina, Z.: 1985, Astron. J. 90, 823, 917.

Larson, S. M. and A'Hearn, M. F.: 1984, Icarus 58, 446.

Lecacheux, J. and LeFevre, O.: 1984, IAU Circ., No. 3957. 
LeFevre, O., Lecacheux, J., Mathez, G., Lelièvre, G., Baudrand, J., and Lemonnier, J. P.: 1984, Astron. Astrophys. 138, L1.

Litvak, M. M. and Kuiper, E. N., Rodriguez: 1982, Astrophys. J. 253, 622.

Lumme, K. and Bowell, E.: 1985, Icarus 62, 54.

Maassberg, H. and Isensee, U.: 1982, ESA SP-187, 21.

Marconi, M. L. and Mendis, D. A.: 1983, Astrophys. J. 273, 381.

Marconi, M. L. and Mendis, D. A.: 1984, Astrophys. J. 287, 445.

Marconi, M. L. and Mendis, D. A.: 1985, (in press).

Marcus, J. N.: 1981, Comet News Service 81 (3), 2.

Marcus, J. N.: 1983, Comet News Service 81 (2), 2.

Marsden, B. G.: 1968, Astron. J. 73, 367.

Marsden, B. G.: 1969, Astron. J. 74, 720.

Marsden, B. G. and Roemer, E.: 1982, in L. Wilkening (ed.), Comets, University Arizona Press, Tucson, p. 707.

Marsden, B. G., Sekanina, Z., and Yeomans, D. K.: 1973, Astron. J. 78, 211.

McDonnell, J. A. M., Alexander, M., Lyons, D., Tanner, W., Anz, P., Hyde, T., Chen, A.-L., Stevenson, T. J., and Evans, S. T.: 1984, Adv. Space Res. 4 (9), 297.

Meier, R. R. and Keller, H. U.: 1985, Icarus 62, 521.

Mendis, D. A.: 1985, in J. Klinger, D. Benest, A. Dollfus, and R. Smoluchowski (eds.), Ices in the Solar System, D. Reidel, Publ. Co., Dordrecht, Holland, p. 487.

Mendis, D. A. and Brin, G. D.: 1977, Moon and Planets 17, 359.

Mendis, D. A., Hill, J. R., Houpis, H. L. F., and Whipple, E. C. Jr.: 1981, Astrophys. J. 249, 787.

Mendis, D. A., Houpis, H. L. F., and Marconi, M. L.: 1985, Fundamentals of Cosmic Physics 10, 1.

Michielsen, H. F.: 1968, J. Spacecraft Rockets 5, 328.

Millis, R. L., A'Hearn, M. F., and Thompson, D. T.: 1982, Astron. J. 87, 1310.

Milman, P. M.: 1976, in H. Elsasser and H. Fechtig (eds.), IAU Colloq. 48, 359.

Mitchell, G. F., Prasad, S. A., and Huntress, W. T.: 1981, Astrophys. J. 244, 1087.

Morbey, C.: 1985, IAU Circ., No. 4034.

Morley, T. A.: 1983, GIOTTO Study Note, No. 46.

Moroz, V. I.: 1982, Pr-736, Space Res. Inst., USSR Acad. Sci., Moscow.

Morris, C. S.: 1980, Int'l Comet Quarterly 2, 69.

Morris, C. S. and Green, D. W. E.: 1982, Astron. J. 87, 918.

Mukai, T. and Fechtig, H.: 1983, Planetary Space Sci. 31, 655.

Münch, R. E.: 1984, ESA Bulletin, No. 38, 42.

Myers, R. V. and Nordsieck, K. H.: 1984, Icarus 58, 431.

Nagel, K., Neukum, G., Dohnanyi, J. S., Fechtig, H., and Gentner, W.: 1976, Proc. 7th Lunar Sci. Conf., 1021.

Newburn, R. L., Jr.: 1979, ESA SP-153, 35.

Newburn, R. L., Jr.: 1981, ESA SP-174, 3.

Newburn, R. L., Jr.: 1983, Adv. Space Res. 2 (12), 111.

Newburn, R. L., Jr.: 1984, Adv. Space Res. 4 (9), 185.

Newburn, R. L., Jr. and Reinhard, R.: 1981, ESA SP-174, 19.

Newburn, R. L., Jr., and Spinrad, H.: 1984, Astron. J. 89, 289.

Newburn, R. L., Jr., and Spinrad, H.: 1985, Astron. J. 90, 2591.

Newburn, R. L., Jr., and Yeomans, D. K.: 1977, NASA TM-78420, App. A.

Newburn, R. L., Jr. and Yeomans, D. K.: 1982, Ann. Rev. Earth Planetary Sci. 10, 297.

Ney, E. P.: 1974, Icarus 23, 551.

Ney, E. P.: 1982, in L. Wilkening (ed.), Comets, University Arizona Press, Tucson, p. 323.

Ney, E. P. and Merrill, K. M.: 1976, Science 194, 1051.

O'Dell, R.: 1971, Astrophys. J. 166, 675.

Oishi, M., Kawana, K., Kobayashi, Y., Maihara, T., Noguchi, K., Okuda, H., and Sato, S.: 1978, Publ. Astron. Soc. Japan 30, 149.

Opal, C. B., Carruthers, G. R., Prinz, D. K., and Meier, R. R.: 1974, Science 185, 702.

Oppenheimer, M.: 1974, Astrophys. J. 196, 251.

Orlov, S. V.: 1935, Komety, ONTI, Moscow.

Parker, L. W.: 1984, ESA SP-224, 65. 
Patashnick, H. and Rupprecht, G.: 1977, Icarus 30, 402.

Patashnick, H., Rupprecht, G. and Schuerman, D. W.: 1974, Nature 250, 313.

Pereyra, Z. M. and West, R. M.: 1984, private communication.

Probstein, R. F.: 1969, in F. Bisshopp et al. (eds.), Problems of Hydrodynamics and Continuum Mechanics, Soc. Industr. Appl. Math., Philadelphia, p. 568.

Rahe, J., McCracken, C. W., Hallam, K. L., and Donn, B. D.: 1976, Astron. Astrophys. Suppl. $23,1$.

Reinhard, R.: 1979, ESA SP-153, 7.

Reinhard, R.: 1981a, ESA SP-164, 221.

Reinhard, R.: 1981b, ESA SP-169, X.

Reinhard, R.: 1982, in P. Veron, M. Festou and K. Kjar (eds.), Proceedings of the ESO Workshop on the Need for Coordinated Ground-based Observations of Halley's Comet, p. 21.

Reinhard, R.: 1984, ESA Bulletin, No. 38, p. 90.

Roemer, E.: 1963, in B. M. Middlehurst and G. P. Kuiper (eds.), The Moon, Meteorites and Comets, University Chicago Press, Chicago, p. 527.

Roser, S.: 1984, Private Communication.

Rüdenauer, F. G. and Steiger, W.: 1984, ESA SP-224, 1.

Sagdeev, R. Z., Avanesov, G. A., Galeev, A. A., Moroz, V. I., Novikov, B. S., and Skuridin, G. A.: 1982, Space Res. Inst. D-308, USSR Acad. Sci., Moscow.

Sagdeev, R. Z.: 1985, Venus-Halley Mission, Imprimerie Louis-Jean, Paris.

Schleicher, D. G. and A'Hearn, M. F.: 1982, Astrophys. J. 258, 864.

Schwarzschild, K. and Kron, E.: 1911, Astrophys. J. 34, 342.

Schwehm, G. H. and Kneissel, B.: 1981, ESA SP-174, 77.

Sekanina, Z: 1976, NASA SP-393, 537.

Sekanina, Z:: 1979, ESA SP-153, 25.

Sekanina, Z.: 1980a, in I. Halliday and B. A. McIntosh (eds.), Solid Particles in the Solar System, D. Reidel Publ. Co., Dordrecht, Holland, p. 237.

Sekanina, Z.: 1980b, in I. Halliday and B. A. McIntosh (eds.), Solid Particles in the Solar System, D. Reidel Publ. Co., Dordrecht, Holland, p. 251.

Sekanina, Z.: 1981a, Ann. Rev. Earth Planetary Sci. 9, 113.

Sekanina, Z.: 1981b, Astron. J. 86, 1741.

Sekanina, Z.: 1981c, ESA SP-174, 55.

Sekanina, Z.: 1982, in L. Wilkening (ed.), Comets, University Arizona Press, Tuckson, p. 251.

Sekanina, Z.: 1983, Adv. Space Res. 2 (12), 121.

Sekanina, Z.: 1984a, Icarus 58, 81.

Sekanina, Z.: 1984b, Astron. J. 89, 1573.

Sekanina, Z.: 1985a, Astron. J. 90, 827.

Sekanina, Z.: 1985b, Astron. Astrophys. 148, 299.

Sekanina, Z:: 1985c, Astron. J. 90, 1370.

Sekanina, Z. and Farrell, J. A.: 1980, Astron. J. 85, 1538.

Sekanina, Z. and Farrell, J. A.: 1982, Astron. J. 87, 1836.

Sekanina, Z. and Larson, S. M.: 1983, Bull, Am. Astron. Soc. 15, 651.

Sekanina, Z. and Larson, S. M.: 1984, Astron. J. 89, 1408, 1446.

Sekanina, Z. and Larson, S. M.: 1986, Astron. J. 91, in press.

Sekanina, Z. and Miller, F. D.: 1973, Science 179, 565.

Sekanina, Z. and Pansecchi, L.: 1977, Astrophys. Letters 18, 61.

Shimizu, M:: 1975, Astrophys. Space Sci. 36, 353.

Shul'man, L. M.: 1969, Astrometr. Astrofiz. 4, 100.

Shul'man, L. M.: 1972, Dinamika Kometnykh Atmosfer - Neutral'nii Gaz, Naukova Dumka, Kiev.

Sicardy, B., Guerin, J., Lecacheux, J., Baudrand, J., Combes, M., Picat, J. P., Lelièvre, G., and Lemonnier, J. P.: 1983, Astron. Astrophys. 121, L4.

Spinrad, H.: 1982, Publ. Astron. Soc. Pacific 94, 1008.

Stebbins, J.: 1910, Astrophys. J. 32, 179.

Tatum, J. B. and Gillespie, M. I.: 1977, Astrophys. J. 218, 569.

Taylor, F. W., Michaux, C. M., and Newburn, R. L., Jr.: 1973, JPL Tech. Report 32-1590, Pasadena.

Thiemann, H.: 1984, ESA SP-224, 71.

Tokunaga, A. T. and Hanner, M. S.: 1985, Astrophys. J. 296, L13. 
Van de Hulst, H. C.: 1957, Light Scattering by Small Particles, J. Wiley, New York.

Verniani, F.: 1973, J. Geophys. Res. 78, 8429.

Wallis, M. K.: 1982, in L. Wilkening (ed.) Comets, University Arizona Press, Tucson, p. 357.

Wallis, M. K.: 1984, Earth, Moon, and Planets 30, 31.

Wallis, M. K., and Hassan, M. H. A.: 1983, in T. I. Gombosi (ed.), 'Cometary Exploration' Central Res. Inst. Physics, Hungarian Acad. Sci., Budapest 2, 57.

Wallis, M. K. and McPherson, A. K.: 1981, Astron. Astrophys. 98, 45.

Weaver, H. A. and Mumma, N. J.: 1984, Astrophys. J. 276, 782.

Weaver, H. A., Feldman, P. D., Festou, M. C., A'Hearn, M. F., and Keller, H. U.: 1981, Icarus 47, 449.

Weissman, P. R. and Kieffer, H. H.: 1981, Icarus 47, 302.

West, R. M. and Pedersen, H.: 1984, Astron. Astrophys. 138, L9.

Whipple, F. L.: 1950, Astrophys. J. 111, 375.

Whipple, F. L.: 1951, Astrophys. J. 113, 464.

Whipple, F. L.: 1976, Nature 263, 15.

Whipple, F. L.: 1980, IAU Circ., No. 3459.

Whipple, F. L.: 1981, ESA SP-169, 101.

Whipple, F. L.: 1982, in L. Wilkening (ed.), Comets, University Arizona Press, Tucson, p. 227.

Wurm, K.: 1943, Mitt. Hamburger Sternwarte 8, 51.

Yamamoto, T.: 1982, Astron. Astrophys. 109, 326.

Yamamoto, T.: 1985, Astron. Astrophys. 142, 31.

Yamamoto, T. and Ashihara, O.: 1984, ISAS Research Note 276, Tokyo.

Yamamoto, T., Nakagawa, N., and Fukui, Y.: 1983, Astron. Astrophys. 122, 171.

Yeomans, D. K.: 1977, Astron. J. 82, 435.

Yeomans, D. K. and Kiang, T.: 1981, Monthly Notices Roy. Astron. Soc. 197, 633.

Young, D. T.: 1984, ESA SP-224, 99.

Zucrow, M. J. and Hoffman, J. D.: 1976, Gas Dynamics, J. Wiley, New York. 